\title{
The Relationship between Rape Survivors' Levels of Distress, Health Profile, Ways of Coping and Measures of the Immune System
}

\section{Prishika Pillay}

$t$ Submitted in partial fulfilment of he requirements for the degree of Masters of Science ( Clinical psychology) in the Schoaf of psychology, University of Natal, Pietermaritzburg.

January 2001 


\section{Declaration}

This thesis was undertaken in the School of Psychology, University of Natal, Pietermaritzburg and unless otherwise stated in the text, represents the author's own work. This thesis has not been submitted to any other university.

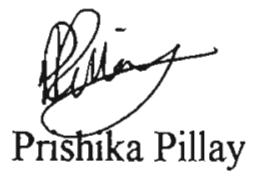

January 2001 


\section{Acknowledgements}

Professor Graham Lindegger, my supervisor, for his encouragement and critical guidance of this thesis.

Dr. B.D. Faulds for his assistance with the statistical analysis.

Dr. Vincent Naicker and Trevor for conducting all the blood tests.

Professor Lawrence Schlebush, for his assistance during the initial stages of this study.

Mr. Jabulani Thwala, for his assistance in translating the questionnaires.

Dr. Kogie Reddy and staff, LANCET Laboratories, for assisting in the drawing of blood samples.

The Districts Surgeons Office in Pietermaritzburg; the South Africa Police - Family violence, child protection and sexual offences unit and the staff of the Crisis Clinic at Mahatma Ghandi Hospital, for their assistance in recruiting rape survivors.

The rape survivors who participated in this study.

Anne Kramers, my friend, for proof reading this thesis.

My mother, Radha, and my sister, Sanoosha, for their care and understanding, my brother-inlaw, Deon for his assistance with graphics and my late father for teaching me commitment and perseverance needed to complete this thesis.

The financial contribution of the Centre for Science Development [CSD] to this thesis is gratefully acknowledged. The views and conclusions in the thesis do not necessarily reflect those of the CSD. 


\section{TABLE OF CONTENTS}

\section{Preface}

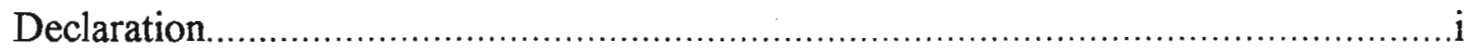

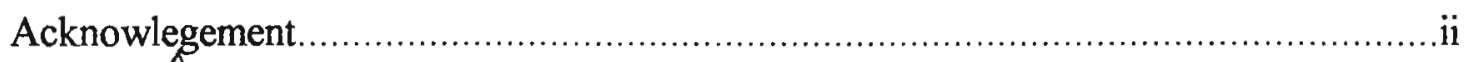

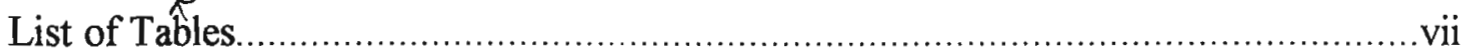

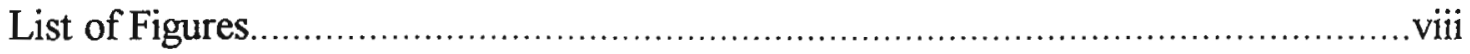

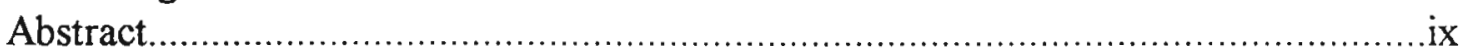

Chapter 1: Introduction............................................................................................1

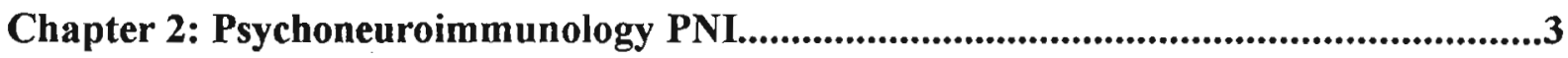

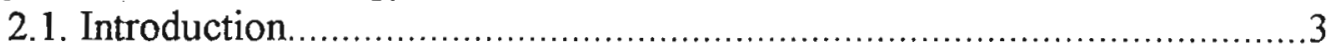

2.2. Evidence for the link between the Central Nervous System and the Immune System.......................................................................... 4

2.3. Connections from the Central Nervous System to the Immune system.....6

2.4. Connection from the Immune System to the Central Nervous System......7

2.5. Psychological Modulation of Immunity ............................................. 10

2.5.1. Acute stressors in the laboratory ..................................... 10

2.5.2. Naturalistic Stressors.................................................. 12

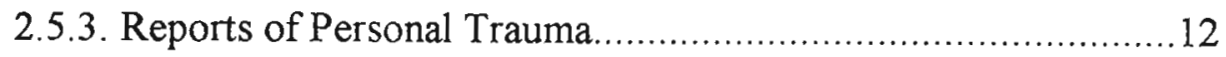

2.5.4. Mood and Anxiety States................................................ 13

2.5.5. Stress Motivation............................................................ 14

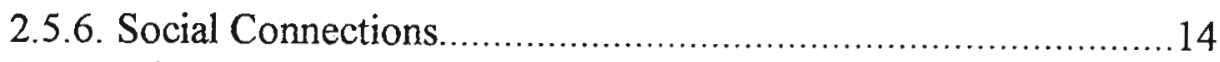

2.5.7. Minor Hassels in community volunteers.............................. 15

2.5.8. Academic Stress...........................................................15

2.5.9. Caregivers of Dementia / Alzeimers patients.........................15

2.6. Impact of Stressors on Infections and Illness................................16

2.6.1. The influence of Stressors on Infection...............................17

2.6.2. Stress and the Severity and Cause of Infectious Disease..........19

2.6.3. Upper Respiratory Infection..........................................20

2.6.4. Herpes Virus Infections.............................................20

2.7. Methodological, Conceptual and Statistical Issues in PNI research..........21

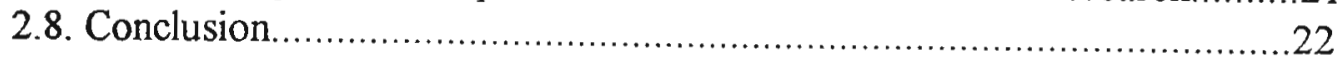

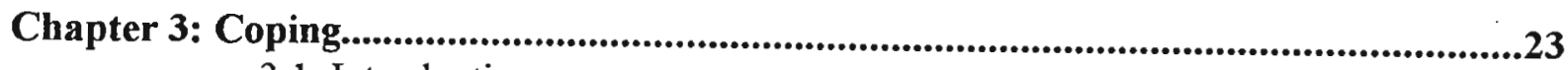

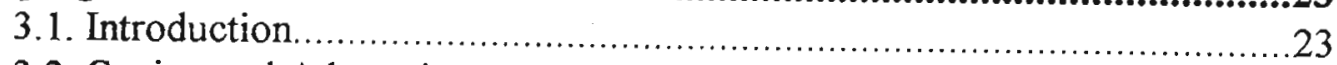

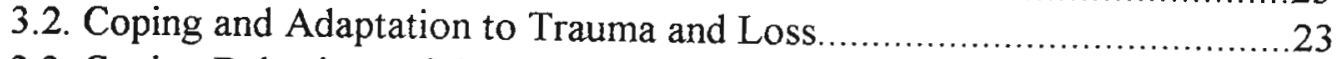

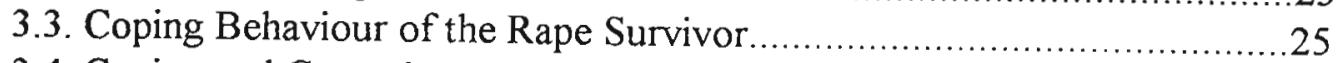

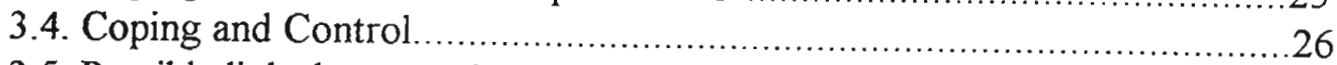

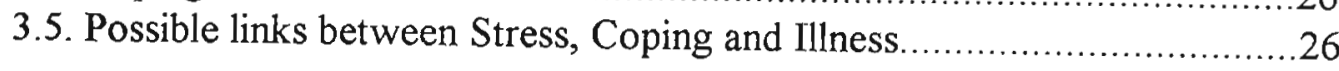

Chapter 4: Rape - A Life Change Event...................................................................27

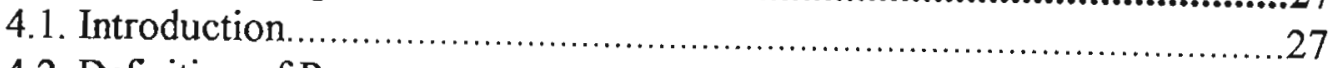

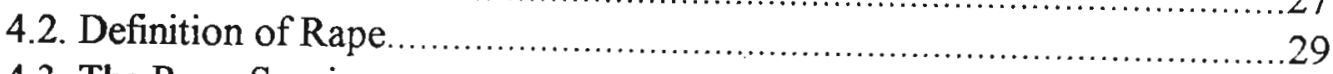

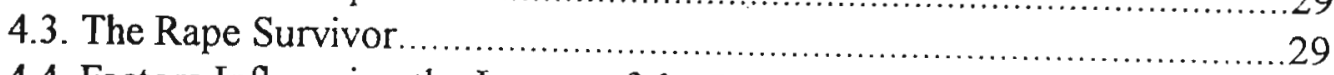

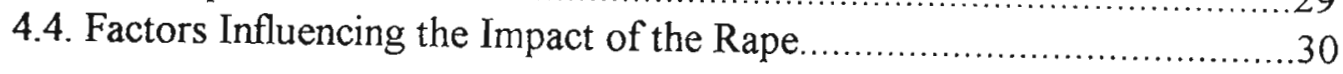




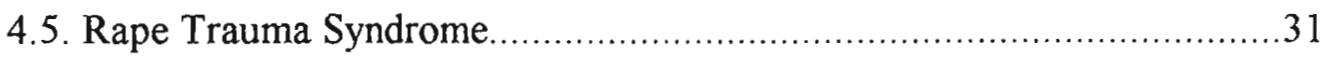

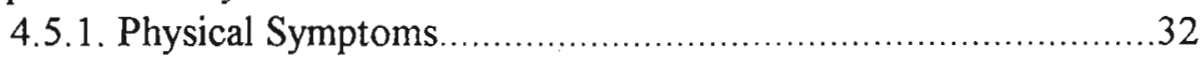

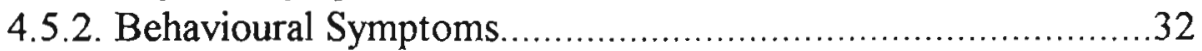

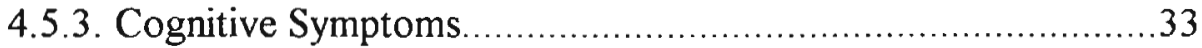

4.5.4. Emotional Symptoms...........................................................34

Chapter 5: Methodolgy................................................................................................................35

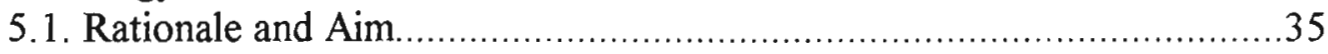

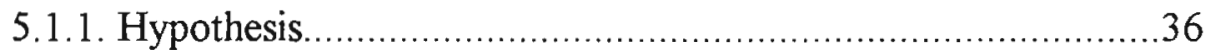

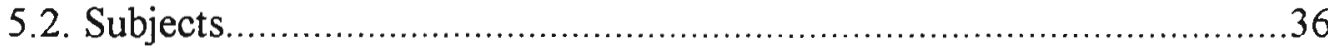

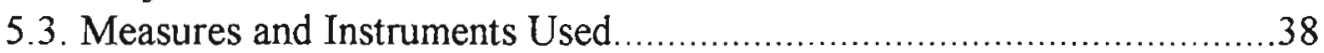

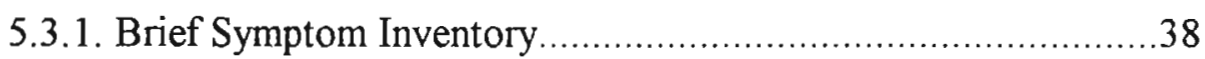

5.3.2. Revised Impact of Events Scale .............................................40

5.3.3. Ways of Coping Checklist......................................................41

5.3.4. Recent Life Change Questionnaire.........................................4 44

5.3.5. Duke University of North Carolina Health Profile.....................44

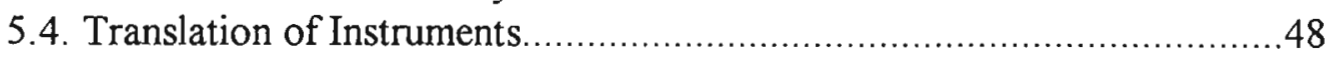

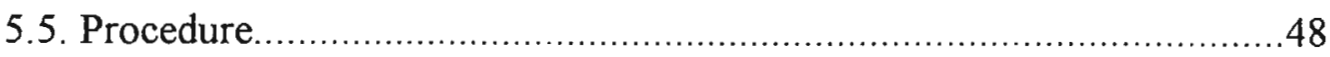

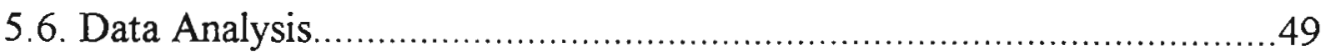

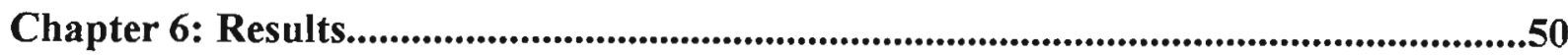

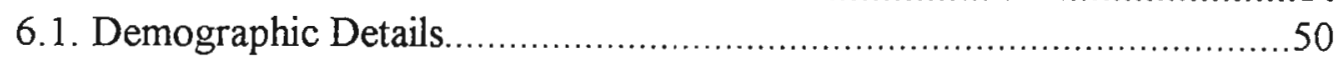

6.2. Analysis Across Time......................................................................52

6.2.1. A summary of the psychological and immune measures for

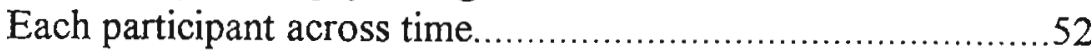

6.2.2. Analysis of Immune Measures across time..............................55

6.2.3. Analysis of Levels of Distress across time................................60

6.2.4. Analysis of Impact of Events across time.....................................63

6.2.5. Analysis of Ways of Coping across time...................................63

6.2.6. Analysis of Health Profile across time......................................65

6.3. The Relationship between Different Variables..........................................66

6.3.1. The relationship between Life Change Events and Immune

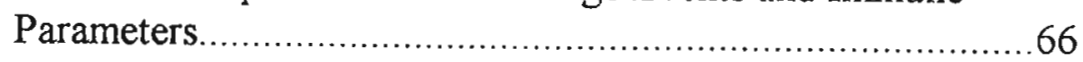

6.3.2. The relationship between Levels of Distress and Immune Parameters. 66

6.3.2.1. relationship between levels of distress and

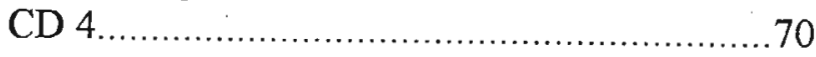

6.3.2.2. relationship between levels of distress and neutrophils.................................................. 70

6.3.2.3. relationship between levels of distress and lymphocytes

6.3.2.4. relationship between levels of distress and CD 8 .

6.3.2.5. relationship between levels of distress and CD 3

6.3.2.6. relationship between levels of distress and eosinophils.

6.3.2.7. relationship between levels of distress and 
monocytes.

6.3.2.8. relationship between levels of distress and white cell count.............................................72

6.3.2.9. relationship between levels of distress and platelets.

6.3.3. Relationship between Ways of Coping and Immune Parameters.

6.3.3.1. relationship between ways of coping and CD 4 ..

6.3.3.2. relationship between ways of coping and lymphocytes.

6.3.3.3. relationship between ways of coping and neutrophils

6.3.3.4. relationship between ways of coping and eosinophils.

6.3.3.5. relationship between ways of coping and CD 8 .

6.3.3.6. relationship between ways of coping and CD 3 .

6.3.3.7. relationship between ways of coping and monocytes.

6.3.3.8. Conclusion

6.3.4. Relationship between the Health Profile and Recent Life Changes.....

6.3.5. Relationship between the Health Profile and Blood Measurements

6.3.5.1. relationship between health profile and CD 4.

6.3.5.2. relationship between health profile and CD 3 .

6.3.5.3. relationship between health profile and CD 8 .

6.3.5.4. relationship between health profile and white cell count.

6.3.5.5. relationship between health profile and neutrophils.

6.3.5.6. relationship between health profile and monocytes.....

6.3.5.7. relationship between health profile and eosinophils.

6.3.5.8. Conclusion

6.3.6. Relationship between Immune Parameters and Recent Life Events

6.3.7. Relationship between Levels of Distress and Coping

6.3.7.1. relationship between levels of distress and acceptance. 
6.3.7.2. relationship between levels of distress and emotional withholding.

6.3.7.3. relationship between levels of distress and wishful thinking.

6.3.7.4. relationship between levels of distress and growth.

6.3.7.5. relationship between levels of distress and problem-solving / help-seeking.

6.3.7.6. relationship between levels of distress and help-seeking.

6.3.7.7. Conclusion.

Chapter 7 .86

7.1. Introduction

7.2. Demographic Details.

7.3. The Relationship between Levels of Distress and Immune Parameters.....87

7.3.1. The levels of Distress across time ........................................87

7.3.2. The Immune parameters.............................................. 89

7.3.3. The relationship between levels of distress and immune parameters across time.................................................91

7.4. The Relationship between Ways of Coping and Immune Parameters...... 94

7.4.1. Introduction............................................................. 94

7.4.2. The Ways of Coping across time........................................94

7.4.3. The relationship between Ways of Coping and Immune Parameters across time

7.5. The Relationship between the Duke-UNC Health Profile and Immune Parameters

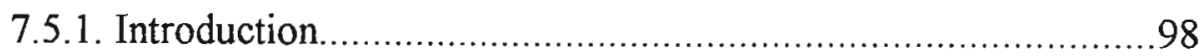

7.5.2. The Health Profile across time........................................ 98

7.5.3. The relationship between the Health Profile and Immune Parameters.

7.6. The Relationship between the levels of Distress and Ways of Coping ...100

7.7. Implications of the Research..................................................... 103

7.8. Limitations of the Research ..................................................... 104

References

Appendix 1 - The Immune System................................................ 120

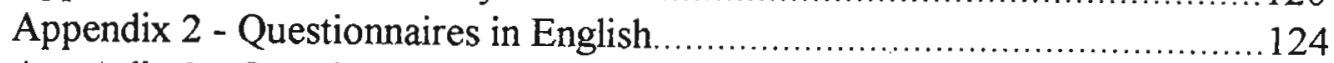

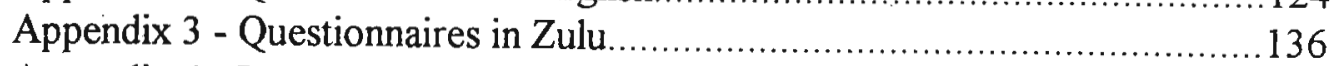

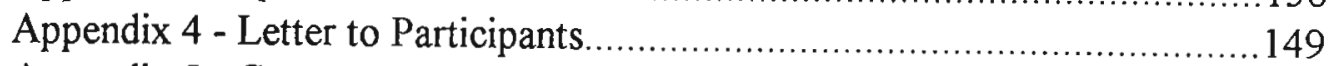

Appendix 5 - Consent Form........................................................... 152

Appendix 6 - Figure 8 ...................................................................... 155

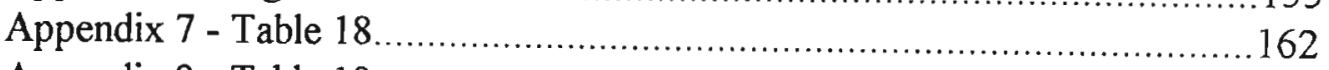

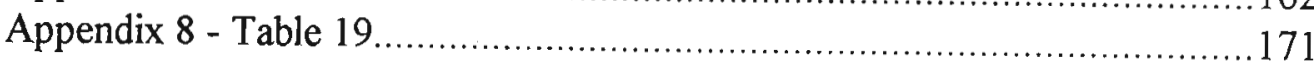




\section{LIST OF TABLES}

Table 1: $\quad$ Sample size at times 1,2 , and 3 .

Table 2a:

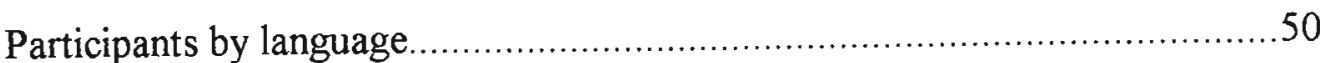

Table $2 \mathrm{~b}$ :

Table 2c:

Table 2d:

Table 2e:

Table 2f:

Table $2 \mathrm{~g}$ :

Table 2h:

Participants by ethnic group....................................................... 50

Participants by religious affiliations................................................... 51

Participants by age ..................................................................... 51

Participants by marital status....................................................... 51

Participants by current employment status........................................ 51

Participants by level of education................................................... 51

Participants by HIV status........................................................ 51

Table 3: Mean blood measures at times 1, 2, and 3, and normative levels of the various blood measures............................................................ 57

Table 4: $\quad$ Relationship between levels of distress and blood measurements $\mathrm{n}=12$, time 1

Table 5: Relationship between levels of distress and blood measurements

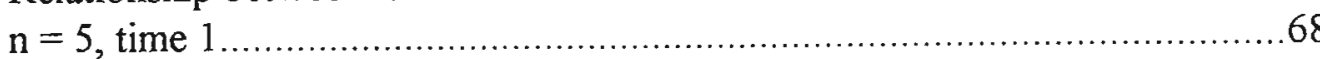

Table 6: Relationship between levels of distress and blood measurements $\mathrm{n}=5$, time 2 .

Table 7: $\quad$ Relationship between levels of distress and blood measurements $\mathrm{n}=5$, time 3

Table 8: $\quad$ Relationship between ways of coping and blood measurements $\mathrm{n}=12$, time 1 .

Table 9: Relationship between ways of coping and blood measurements $\mathrm{n}=5$, time 1

Table 10: Relationship between ways of coping and blood measurements $\mathrm{n}=5$, time 2

Table 11: Relationship between ways of coping and blood measurements $\mathrm{n}=5$, time 3

Table 12: Relationship between Duke Health Profile and blood measurements $\mathrm{n}=12$, time 1

Table 13: Relationship between Duke Health Profile and blood measurements $\mathrm{n}=5$, time 1 .

Table 14: Relationship between Duke Health Profile and blood measurements $\mathrm{n}=5$, time 2 .

Table 15: Relationship between Duke Health Profile and blood measurements $\mathrm{n}=5$, time 3

Table 16: Relationship between Levels of Distress and Coping $\mathrm{n}=5$, time 2 .

Table 17: $\quad$ Relationship between Levels of Distress and Coping $\mathrm{n}=5$, time 3

Table 18a-v: Psychological Measures of each participant across time 1,2 and 3.

Table 19: $\quad$ Mean Scores and Standard Deviations of Psychological Measures across time 1,2 and 3 . 


\section{LIST OF FIGURES}

Figure 1: Illustration of the Brain-Immune System Connections ..............................

Figure 2: $\quad$ Behavioural and Biological pathways linking stress to the onset of

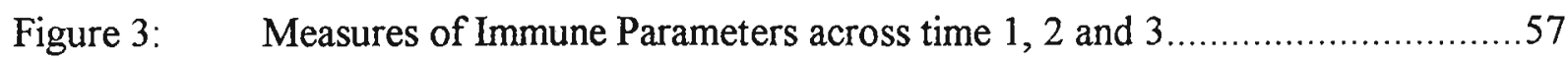

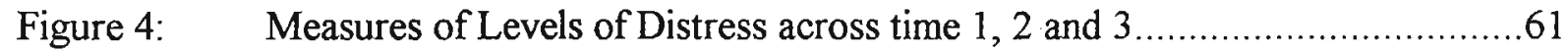

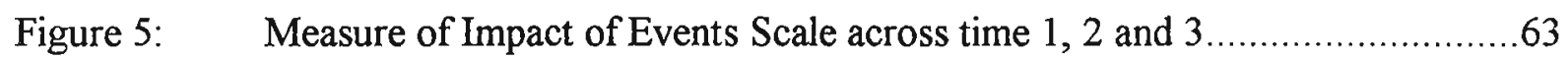

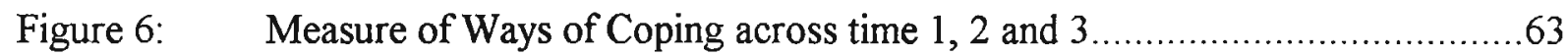

Figure 7: $\quad$ Measure of the dimensions of Health Profile across time 1,2 and 3..........65

Figure 8: $\quad$ Participants' Immune Parameters across time 1, 2 and 3....................... 155 


\begin{abstract}
This research aims to investigate the relationship between rape survivors levels of distress, coping style, health profile and immune system. Psychoneuroimmunology, an interdisciplinary field of study, is employed as a framework to understand the relationship between the levels of distress, coping style, health profile and the immune system A sample of 36 rape survivors was initially recruited for this study. However data collected from a sample of 12 female rape survivors was selected for this research. These participants completed questionnaires measuring levels of distress, impact of the event, recent life changes and health profile at time 1 ( 5 days post rape), time 2 ( 15 days post rape) and time 3 ( 35 days post rape). the immunological measures included CD 3, CD 4, CD 8, neutrophils, monocytes, eosinophils, lymphocytes, white cell count, platelets and HIV (time 1 only).
\end{abstract}

The results revealed significant relationships between levels of distress and immune parameters; health profile and immune parameters; ways of coping and immune parameters and levels of distress and immune parameters. Significant differences were obtained for CD $45(p=0.039)$ between time 1 and time 2 , as well as between time 2 and time 3 . A significant difference $(p=$ 0.039 ) was noted for platelets between time 2 and time 3 . The levels of distress were raised at time 2. Observation of each participant revealed no dramatic changes across time 1,2 and 3 .

When a person is subjected to rape, heightened levels of distress are experienced 15 days post rape. Rape survivors experience changes in the levels of distress, health profile, ways of coping and immune parameters over a 35 days post rape period. 


\section{INTRODUCTION}

Rape is a traumatic event which has devastating consequences for the survivor. These consequences can either be psychological or physical. Irrespective of the consequences the survivor is faced with, he or she would try to cope with them in order to maintain equilibrium. Thus rape places a demand on the individuals' internal and external resources. Rape is not uniformly stressful to all people. The experience of stress can result in different consequences which include physical symptoms such as increased blood pressure, headaches, fatigue; behavioural symptoms such as increased smoking, alcohol use, social withdrawal and appetite changes and emotional symptoms such as feeling overwhelmed, loss of control, anxiety and confusion (Leonard \& Miller, 1995). Stressful life events are commonly believed to alter immunity and hence susceptibility to immune system-mediated disease (Cohen \& Herbert, 1996). Although studies have repeatedly shown that stressors affect the immune system, extensive variability exists in both baseline immune measures and the individual immune responses to stressors. Rape can impose demands which exceed the individual's ability to cope thus eliciting a psychological stress response composed of negative cognitive and emotional stress. It is these responses that are thought to influence immune function through their effects on behaviour and coping such as changes in health practices (Cohen \& Williamson, 1991;Turner-Cobb \& Steptoe, 1996) and neuro-endocrine responses (Cohen \& Herbert, 1996).

When individuals' resources are insufficient to meet the demand being placed, they experience a stress response. The person experiencing these symptoms and the subjective feeling of being stressed will attempt to manage the situation and reduce the stress symptoms by using coping strategies. There are two broad categories of coping styles namely problem-focused coping and emotional-focused coping (Lazarus, De Longis, Folkman \& Gruen, 1985).

Cumulative stress scales used widely in the literature, for the most part, tap levels of stress within the normal range of variations that people experience in day to day life (Glaser \& Kiecolt-Glaser, 1994). Impact of severe events would provide the fairest test of a stress-disease reaction (Cohen \& Williamson, 1991). A literature scan revealed that, to date, no research within this arena has considered the relationship between rape and the immune system. Most studies have concentrated 
on stressful daily events, examination stress and laboratory stress. It is also important to note that most studies reveal little about the nature of stressors that put a person at risk for disease; timing of the stressor relative to exposure and disease onset; and psychological and biological characteristics that moderate these effects.

The present study attempts to investigate the relationship between the distress of rape, ways of coping, health profile and cell-mediated immunity. Chapter 2 presents a review of the literature in psychoneuroimmunology, a theoretical framework for this study. This is followed by a presentation of literature on coping (Chapter 3). This chapter highlights the literature on coping and the links between coping and the immune system. Chapter 4 provides a review of literature on rape, in order to illustrate the traumatic nature of rape and the consequences thereof. Chapter 5 outlines the research methods and procedures used in this study. This is followed by a presentation of the research findings (Chapter 6), and Chapter 7 provides a discussion of these findings, limitations of the study and implications for future research. 


\section{PSYCHONEUROIMMUNOLOGY}

\subsection{Introduction}

Through the ages and across cultures, wise physicians have linked psychic and bodily well-being and have observed that illness often followed stressful life events. In 1964, George Solomon coined the term "psychoimmunology" to describe this new science. Interest in this area sparked in the mid 1970's when Ader and Cohen demonstrated that immunological responses could be classically conditioned and since then has exploded into a genuine field of interdisciplinary research. This finding implicates neuro-endocrine pathways in the relationship between stressors and immunological responses. Hence the realization that the immune system and nervous system do not operate autonomously, as had previously been supposed. This culminated in the birth of a more appropriate term psychoneuroimmunology (PNI) (Zeller, 1996). Psychoneuroimmunolgy aims to understand the relationships among the psyche, or mind, the neuroendocrine system and the immune system, as well as the role of these relationships in maintenance of health or vulnerability to illness (Aldwin, 1994).

An assumption or premise of psychoneuroimmunology at least in relation to any clinical implication, is that all disease (including mental illnesses and medical conditions not ordinarily considered "psychosomatic") is multi-factorial in origin, the result of interrelationships among the genetic, endocrine, nervous and immune systems and behavioural-emotional factors (Salk, 1962).

Solomon (1990) expressed that within the field of PNI, the need now is to focus on specific aspects of the field, which extends from the underlying molecular biological mechanisms that mediate between the central nervous system and the immune system, to the behavioural correlates, which include clinical and experimental, and to particular features of the immune response. Researchers are making earnest attempts to explore these avenues. Being an interdisciplinary field undoubtedly results in investigations of psychoneuroimmunology being more difficult since both behavioural science and immunology are growing exponentially in knowledge and complexity (Solomon, 1990). 


\subsection{Evidence for the link between the Central Nervous System and the Immune System}

Work with animals has provided evidence for the link between the immune system and the central nervous system. There is sufficient evidence for the following:-

- nerves connecting the Central Nervous System (CNS) and the immune system (Felten, Felten, Carlson, Olschowka \& Livnat, 1985)

- neuroendocrine-induced alterations of specific immune functions (Maier \& Watkins, 1998).

- the existence of chemicals called cytokines, that are produced by the immune system, Cytokines are capable of crossing the blood-brain barrier, and altering central nervous system functioning (Dunn, 1995).

A leap forward in establishing the interaction between the CNS and the immune system was accomplished by researchers who worked with animal models to demonstrate that changes in the immune system could be induced by classically conditioned stimuli (reviewed in Ader \& Cohen, 1993). It is apparent from the work in this field that there are overlapping interests in studies carried out on humans and animals. Although the emphasis placed on human research is different, both animal and human research in PNI compliment each other. Examples of overlap in focus include classical conditioning of human immune response (Ader \& Cohen, 1993) and demonstrations of immune-system effects on the central nervous system as reflected in human performance (Smith, Tyrrell, Al-Nakib, Coyle \& Donovan, 1988 ).

Various differences between animal and human studies exist. Human studies are primarily concerned with the impact of behaviour and personality traits and states on central nervous system and immune responses. The major foci of human studies pertain to establishing whether there is an association between psychological traits and states and immunity; to investigate what the biological and behavioural pathways are that might be responsible for such relations; and to assess whether psychologically induced changes in immunity are responsible for changes in susceptibility to immune system-mediated disease.

It is relevant to overview some of the human and animal research evidence in this field. The evidence shows that various stressful situations effect the immune and central nervous system. 
Human studies have focussed on a range of psychological variables including placebo effects, psychiatric disorders, bereavement, academic stress, studies looking at divorce and the stress of caring for family members with alzheimers disease (AD). There appears to be no strong evidence for direct effects of placebos on immune function, but placebo effects via psychological states such as anxiety may explain some of the medical effects. Research conducted on psychiatric disorders substantially suggests abnormalities of immune function in the mentally ill, however the primary cause is not clear. Research in this field raises some important questions like, can immune function be altered by brain dysfunction? or can psychiatric disease be caused by immune system defects?.Bereavement has been one of the most frequently studied areas. Repeated studies reveal a decreased lymphocyte function in bereaved individuals. Stress has also been studied by Glaser \& Kiecolt-Glaser (1994) who conducted various studies using medical students and monitoring them around the time of examinations. Results of these studies showed examination-related decreases in NK (natural killer)-cell activity and increased titres of antibodies to herpes simplex virus, Eptein-Barr Virus and cytomegalovirus. It is worth noting that the increased antibody titres are interpreted as having resulted from reactivation of latent viruses by the stress of the examination. It is also debatable as to whether or not examinations are comparable to other life stresses (Leonard \& Miller,1995). These studies will be reviewed in further detail to clearly outline how behaviour drives central nervous system and immune system responses.

Maier, Watkins \& Fleshner (1994) suggest that the immune system serves as a diffuse sensory organ to provide the brain with diverse input. Hence indicating the ability of the immune system to control neural function and the ability of the central nervous system to control the immune system. This existence of neural-immune interactions facilitates the entrance of behaviouralpsychological events to enter the network. Maier et al. (1994) note that if neural processes regulate immune processes, then there is a pathway by which psychological factors could impact on immunity. Conversely, if immune processes alter neural function, then they can also potentially impact on behaviour, emotion and thought. This leads to the recognition of PNI, as mentioned earlier which, is the study of complex interactions between neural, immune and behavioural processes. Since these systems can impact on each other it is necessary to shead some light on the connection between these systems. 


\subsection{Connections from the Central Nervous System to the Immune System}

It is salient to consider these connections as they provide the basis of how the brain regulates the immune system. In order for this regulation, the brain would have to make physical contact with the immune system and alterations in the activity of these connections would undoubtedly affect the course of immune responses to antigens. The brain has the ability to control peripheral organs and processes through the peripheral nervous system. Research over many years has revealed that the sympathetic nervous system innervates organs involved in the immune system such as the thymus, bone marrow and spleen and lymph nodes (Maier et al., 1994). The terminals of the sympathetic nerves release the catecholamines and norepinephrines. This can be sensed by immune organs and cells which contain catecholamine receptors. Physical contact between the brain and immune system also occurs through the terminals of sympathetic nerves in these immune organs which make contact with lymphocytes themselves. Felten \& Felten, (1991). state that these contacts have the ultrastructural features of synaptic contacts.

Communication by the brain with peripheral organs also occurs when the brain releases factors that cause endocrine glands to secrete hormones into the circulation, thus enabling the hormones to reach the various organs and bind to hormone receptors on the organs. The release of glucocorticoids serves as a good example to illustrate this communication pathway. Many of the bodily effects of stress are produced by steroid hormones called glucocorticoids. These hormones are released from the outer portion (cortex) of the adrenal glands. Stress is often characterised by the existence of high levels of these hormones in the blood. The paraventricular nucleus of the hypothalamus synthesizes and releases corticotropin releasing hormone into the portal blood. This stimulates the anterior lobe of the pituary gland to synthesise and release adrenocorticotrophic hormone into the blood. This hormone in turn stimulates the adrenal gland to release glucocorticoids. The connections are indicated in Figure 1.

The involvement of CNS circuitry in the modulation of immune responses was tested directly in lesioning studies. Discrete lesions in hypothalamic areas (particularly anterior / pre-optic regions), limbic forebrain structures (amygdala, hippocampus, septum ), brain stem autonomic or reticular regions (raphe nuclei, reticular formation areas, parabrachial nuclei) and the cerebral cortex result in alterations in specific immune reactivity in the direction of either enhancement or suppression 
that can be observed either transiently or chronically depending on the site and extent of the lesion (Roszman, Cross, Brooks \& Markesbery, 1985; Roszman \& Carlson, 1991).

There is consensus from various studies that the anterior hypothalamus is involved either directly or indirectly in the stimulation of both humoral and cell mediated immune functions (Felten, Cohen, Ader, Felten, Carlson \& Roszman, 1991). This suggests that the anatomical features are structured such that the brain could control immune cells and organs similarly to how it controls other peripheral structures. There is evidence suggesting the participation of the brain in the regulation of the immune system, (Glaser \& Kiecolt-Glaser, 1994; Rabin, 1999) With respect to the autonomic system, chemical destruction with 6 hydroxydopamine can impair some aspects of immune function (Lvnat, Felten, Carlson, Bellinger \& Felten, 1985, cited in Maier et al., 1994).. Maier et al. (1994) highlight the point that destruction or stimulation of neural pathways that are connected to the immune system do alter the function of the immune system hence revealing that the connection between the central nervous system is of real significance, and not merely an anatomic curiosity. Similarly, Blalock, Smith \& Meyer (1985) found that blocking the hormone receptors on lymphocytes alters the course of immunity.

\subsection{Connections from the Immune System to the Central Nervous System}

The immune system response occurs outside the nervous system in the periphery in response to peripheral antigens. In order for the brain to take part in the regulation of this response, it must receive information that an immune response is in fact occurring. The immune response is a complex reaction which extends over a number of days and involves various cell types and mediators ( Maier et al. 1994). Thus it must be essential for the central nervous system to receive detailed information about the course of the response. Maier et al.(1994) further state that both the electrical and chemical activity of the brain change as immune responses occur.

The cells produced by the immune system, such as T cells and B cells have only limited access to the brain, because of the blood-brain barrier. Cells of the immune system such as macrophages are activated in response to infection. These cells release cytokines which are critical signals to the central nervous system ( Maier \& Watkins, 1998). Cytokines include interleukins, the interferons, tumor necrosis factors and a variety of cell growth stimulating factors. Cytokines have 
numerous effects which might be related to the physiology of infections. One such example is interleukin-1 (L-1), which is capable of activating the hypothalamic-pituiatry-adrenal (HPA) axis as well as the cerebral and peripheral noradrenergic systems (Leonard \& Miller, 1995; Maier \& Watkins, 1998). The most pronounced changes are in norepinephrine in the hypothalamus (Dunn, 1992) and in serotonin in the hippocampus ( Linthorst, Flachskamm, Holsboer \& Reul (1994). Interleukin-1 can cross the vascular endothelium in regions of the brain where the barrier is weak or absent (Katsuura, Arimura, Koves \& Gottchall, 1990 in Maier et al., 1994). Interleukin-1 can stimulate peripheral nerves, such as the vagus, that send afferent input to the brain (Watkins et al., 1994 in Maier, 1995 \& Maier, Goehler, Fleshner \& Watkins - in press), hence suggesting that the immune system may truly act as a sensory organ conveying information to the brain. This is illustrated in Figure 1. The activation of the HPA axis associated with immune responses gives further weight to the above suggestion. 


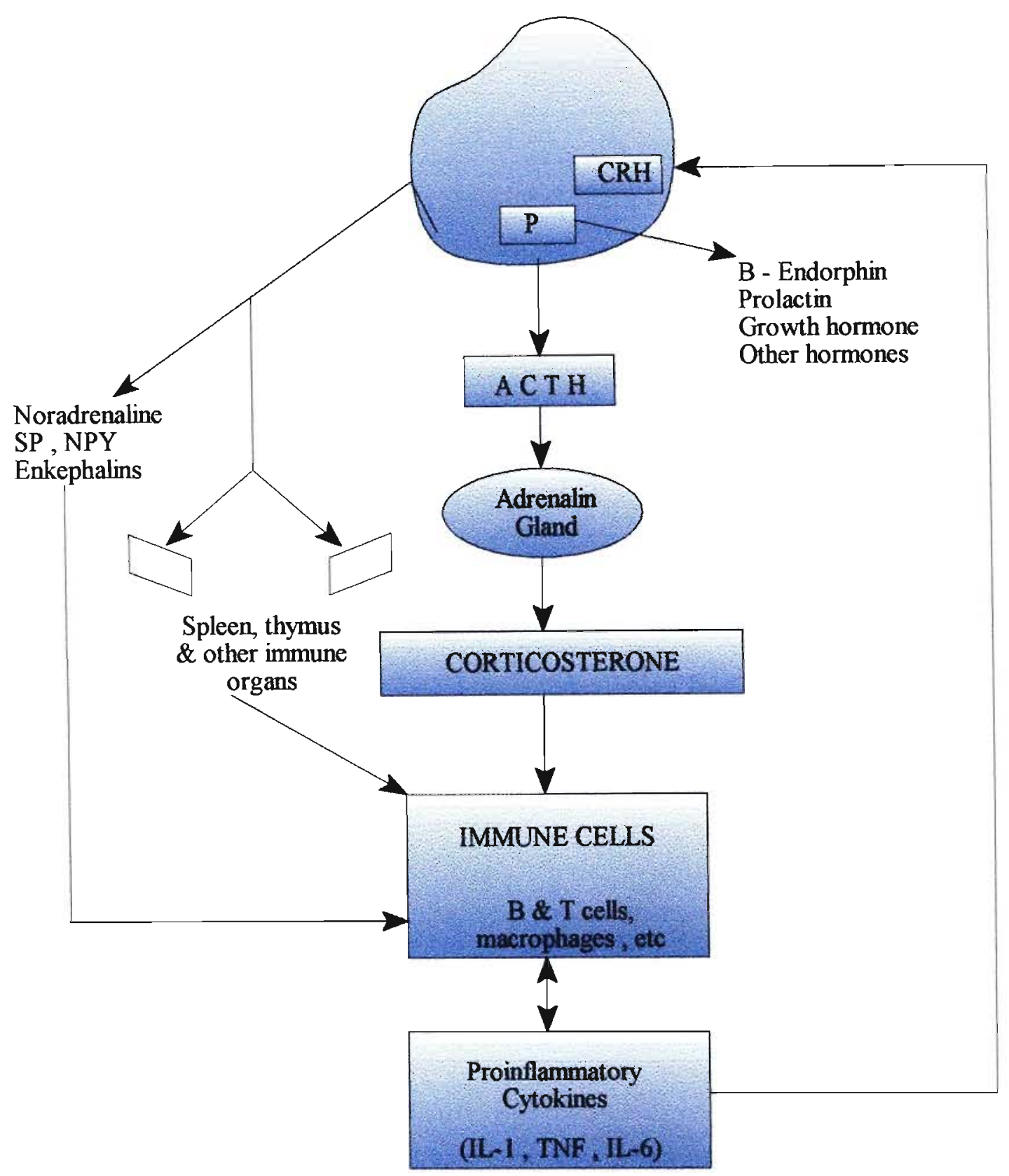

Figure 1 : Illustration of the Brain - Immune System connections.

The ability of the brain to alter immune functioning via endocrine pathways and the autonomic nervous systemis indicated as well as the effects of peptides \& cytokines on the immune cells and the brain, (adapted from Dunn, 1995; Maier \& Watkins, 1998).

$\mathrm{CRH}$ - corticotropin releasing factor $P$ - Pituitary Gland ACTH - adrenocorticotropic hormone TNF - tumor necrosis factor
SP - Substance P

NPY - neuropeptide $Y$

IL-1- interleukin 1

IL-6- interleukin 6 


\subsection{Psychological Modulation of Immunity}

Psychological events are capable of altering neural activity. Neural events are capable of making contact with the immune system as illustrated in Figure 1. Hence there is suggestion that psychological events could modulate immunity.

The studies reviewed below represent the body of research providing measures testing various aspects of psychoneuroimmunology. Support for the linkages will be demonstrated in some cases utilising various population groups. Psychological factors that influence immunity have the potential to influence the onset and progression of immune system-mediated disease because disease occurs when host defences are compromised or unable to recognise the foreign material (Cohen \& Herbert, 1996).

\subsubsection{Acute stressors in the Laboratory}

O'Leary (1990) describes acute stress as being the stress associated with a single event, even though the duration of anticipatory or consequent distress may vary. There have been several studies focusing on the effects of acute psychological stressors. Most of these acute stressors have lasted 5 - 20 minutes for example, speech task and mental arithmetic. Reports on the most consistent immune changes subsequent to stressor exposure suggest an increased natural killer and suppresor / cytotoxic $\mathrm{T}$ cell number and decreased proliferative response to mitogens, particularly PHA (phytohaemaglutinin). (Herbert et al, 1994 ; Manuck, Cohen, Rabin, Muldoon \& Bachen,1991; Naliboff, Benton, Solomon, Morley \& Fahey, 1991, Zakowski, McAllister, Deal \& Baum, 1992).

Herbert et al., (1994) conducted a study which revealed that immune changes in both cell numbers and function can be found as soon as five minutes after the onset of the stressors. Immune parameters return to a resting level in approximately one hour following cessation of the stressor (Kiecolt-Glaser, Cacioppo, Malarkey \& Glaser, 1992), however there is evidence indicating that natural killer activity remains depressed for as long as 48 hours (Sieber, Rodin, Larson, Ortego \& Cummings, 1992). Cohen \& Herbert (1996) state that stress-elicited immune responses found in the laboratory are at least partially attributable to a dispositional style of responding to stress. Data indicating that stress-induced immune responses are reliable across time and tasks clearly 
suggests this ( Boyce, Chesney, Alkon, Tschann, Adams, chesteman, Cohen, Kaiser, Folkman \& Wara,1995; Marsland, Manuck, Fezzari, Steward \& Rabin, 1995). Cohen \& Manuck (1995) claim that the existence of dispositional immune reactive styles allows the possibility that greater immune reactivity may place people at risk for stress-elicited immune related disease. KiecoltGlaser et al., (1992) summarised 9 studies where stressors included mathematical tasks, the stroop test, electric shock, time limited recall of positive and negative emotional experiences, time limited viewing as well as recall of a gruesome film. They concluded that short-term stressors can produce transient immunological alterations, with some facets of the immune response appearing more susceptible than others. They note that consistent changes were noted in an increase in natural killer cell numbers and a decrease in mitogen response particularly for concanavalin $\mathrm{A}$. Alterations in CD8 numbers were inconsistent, but appeared to increase in studies with longer and/or more intensive stressors.

Laboratory studies (Glaser et al.,1994) reveal correlations between sympathetic and immune response, which are as follows: individuals characterised by high sympathetic nervous system (SNS) activation in the light of acute stressors also showed the largest immune changes. High SNS activity is characterised by increases in blood pressure, heart rate and SNS hormones such as epinephrine and norepinephrine (Glaser et al., 1994). In situations where there is no sympathetic reactivity revealed, no change in immune system parameters was noticed (Bachen, Manuck, Cohen, Muldoon \& Raible, 1995; Herbert et al., 1994; Zakowski et al., 1992). These correlations between sympathetic and immune response suggest that stress-elicited SNS response may derive the immune changes. This evidence however is only correlational and does not provide a causal chain. Even though studies of acute laboratory stress provide valuable information, various questions are raised and remain unanswered, for example can acute stressor effects found in the laboratory resemble more chronic real life stressful events.

It is worth considering possible mechanisms for changes due to short-term stressors. KiecoltGlaser et al. (1992) state that immunological changes observed following short-term stressors resemble those changes elicited after the administration of epinephrine injection. Crary, Hauser, Borysenko et al., ( cited in Kiecolt-Glaser et al.,1992) have noted increased percentages of NK cells, decreased blastogenic responses to mitogens (PHA, Con A \& pokeweed) as well as 
decreased percentages of CD4 cells, and no changes in total T cells and monocytes.

Stone, Porter \& Neale (1993) found that subjects exposed to an experimental stressor (mental task) had reduced lymphocyte proliferative responses to mitogens in whole blood culture for at least an hour after the stressor exposure. Literature on acute naturalistic stressors, such as rape does not appear in the literature.

\subsubsection{Naturalistic Stressors}

Examination stress studies are one of the few naturalistic stressors studied in humans. Stone, Neale, Cox, Napoli, Valdimarsdottir \& Kennedy-More (1994) conducted a study of the role of positive and negative daily events in humoral immunity. Positive events included accomplishing a goal, experiencing a good interaction with a significant person and negative events include losing keys and having arguments. The findings from the above mentioned studies by Stone et.al. (1994) revealed that the reporting of more desirable events was concurrently related to greater secretary immunoglobulin A (SIgA) antibody production, and the reporting of more undesirable events was related to less desirable events, were also associated with increases in sIgA production over 2 subsequent days. Evidence for the role of day-to-day events in immune regulation is provided by these data, as well as the beneficial role that positive events play in health outcomes.

Long-term stressful events lasting months or even years have a potential to influence the immune system (Cohen \& Herbert, 1996). This has been indicated by the set of studies assessing stress effects on residents of the area surrounding the Three Mile Island (TMI) nuclear power plant (This area was the site of a serious accident in 1979). McKinnon, Weisse Reynolds, Bowles, Baum (1989) pursued a study almost 10 years after the accident and found more antibody to herpes virus in TMI residents that in demographically matched control group residents, suggesting lower cellular immune competence in the TMI residents.

\subsubsection{Reports of Personal Trauma}

Pennebaker, Kiecolt-Glaser \& Glaser (1988) asked 50 healthy undergraduates to write about either traumatic experiences or superficial topics for 4 days. The results suggested that confronting traumatic experiences had positive effects on lymphocyte activity and health 
outcomes.

\subsubsection{Mood and Anxiety States}

Herbert \& Cohen (1993) conducted a meta-analysis of literature pertaining to the relation between parameters of the immune system and negative mood states. Depression is a psychological concomitant of various stressors, particularly those related to social loss and disruption (O'Leary, 1990).

Herbert \& Cohen (1993) found that depressed mood in non-clinical samples is associated with decreased proliferative responses to mitogens and decreased natural killer activity (NK). Clinically depressed individuals were found to have lowered proliferative response to PHA, Con A and pokeweed mitogen; lowered NK activity; higher numbers of circulating white blood cells (primarily neutrophils and monocytes); and lowered numbers of $\mathrm{NK}, \mathrm{B}, \mathrm{T}$, helper $\mathrm{T}$ and suppressor/cytotoxic T cells.

Very few studies have investigated relations between anxiety and immunity. Anxiety is a state often associated with stress (Linn, Linn \& Jensen, 1981). Herbert \& Cohen (1993) conducted a study to determine the association between levels of anxiety in chronically ill patients and their immune functioning. Although these findings are not very relevant to this study, they are worth considering since valuable inferences and deductions might be made. There is no research at present looking at the immune impact of anxiety experienced during trauma or acute stress. Results obtained by Herbert \& Cohen (1993) suggest that more anxious patients have less cellular or T-cell reactivity as measured in vitro by mitogens and mixed lymphocyte culture but more likelihood of multiple positive in vivo tests of cellular immunity.

There is a considerable amount of interest in the role that negative affect plays in the onset and progression of physical illness. Negative affect pertains to undifferentiated subjective distress. Watson (1988), ( in Cohen, Doyle, Skoner, Fireman Gwaltney \& Newson ,1995) claims that negative affect subsumes a broad range of aversive mood states, for example anxiety, hostility and depression, that form a general distress factor. This can be measured either as a transient fluctuation in mood (state) or as a stable individual difference in affective level ( trait). 


\subsubsection{Stressed Motivation}

In 1989 McClelland investigated various subject populations which included college students, prisoners, adults in a health plan, people with type I diabetes and upper respiratory infections. He suggested that a stressed power motive syndrome is associated with sympathetic activation, release of stress hormones, depressed immune functions and susceptibility to infectious diseases. Many researchers have raised concern about the reliance on secretary IgA (Stone et al., 1987; Jemmott \& McClelland, 1989). Some research has used other immune measures such as T4 cells (McClelland, Patel, Brown \& Kelner, 1991) \& NK cell activity (Jemmot et al, 1990). McClelland, Floor, Davidson \& Saron (1980) assessed 40 male college students on need for power, recent negative life events involving power motives, and inhibition of motive expression. Subjects with inhibited power syndrome reported more frequent and more severe illnesses. Baseline measures of epinephrine, norepinephrine and salivary IgA were related to self reported illness experience as a function of stress motives. Subjects with stressed need for power showed higher urinary epinephrine and lower salivary IgA. Higher epinephrine was associated with lower sIgA, and lower sIgA with reports of more frequent illness. The findings clearly suggest that a strong need for power, if inhibited and stressed, leads to chronic sympathetic overactivity which has an immunosuppressive effect and increased illness susceptibility.

\subsubsection{Social Connections}

Adler \& Matthews (1994) suggest that the degree to which an individual is associated with others may also influence health. These authors also report that individuals with more support in the context of stressful experiences are less likely to engage in health-damaging behaviours. Social connections can also provide support for engaging in health promoting behaviours. According to Aaronson, 1989 \& Treiber et al, 1991 (cited in Adler and Matthews ,1994), individuals who report receiving more support for given health related behaviours are more likely to engage in those behaviours. Studies of loneliness, separation and divorce, perceptions of support and disclosure of traumatic events aid in elucidating the impact of interpersonal relationships on immunity and immune system mediated illness. There is sufficient evidence that indicates that interpersonal relationships have health related consequences. Much of the persuasive evidence comes from epidemiological studies that reveal greater morbidity and mortality in people with fewer close 
relationships (Cohen \& Syme, 1985, in Kiecolt-Glaser \& Glaser, 1988).

\subsubsection{Minor Hassels in Community Volunteers}

Levy, Fernstrom, Herberman, Whiteside, Lee, Ward \& Massoudi (1991) conducted a study on community volunteers ranging in age from 18 to 45 years. Stress was measured using the Kanner scale and immune measures included functional (NKCA) and enumerative (percent T, B and NK cell) assays and a measure of Beta-endorphin. The health outcome was derived from a weekly comprehensive self-report for morbidity (days of infectious acute disease : total \& upper respiratory infections). This research showed that lower NK cell activity was associated with higher illness morbidity.

\subsubsection{Academic stress}

Kiecolt-Glaser \& Glaser (1987) conducted several studies investigating academic stress. They found that examinations were associated with increased distress and higher levels of self-reported illness. Significant influences of stress on immunity were found using an extensive battery of immune assays. Results revealed that in the examination period, students had higher EBV VCA (Epstein-barr virus)) antibody (Ab) titres; lower specific memory T-cell killing; negative leukocyte migration inhibition factor (LIF) activity contrasted with positive LIF during the low stress periods; decreased con A induced Interferon-gamma production by lymphocytes, and elevated intracellular cyclic AMP. Other studies reveal consistent results (Leonard \& Miller, 1995), with examination-related decreases in NK-cell activity also being noted.

\subsubsection{Caregivers of Dementia / Alzeimers Patients}

Studies on caregivers of Alzheimers disease $(\mathrm{AD})$ provide mixed results for the impact of the chronic stress on immune sysrem functioning. Kiecolt-Glaser,Glaser, Shuttleworth,Dyer,Ogrocki \& Speicher (1987) discovered that care-giving was associated with distress and elevated levels of herpes virus antibody. When compared to a low stress control group, the only difference noted between the caregivers and the low stress control group was in a variety of health behaviours thought to affect cellular immunity. A similar study by Irwin, Brown, Patterson, Hauger, Mascovich \& Grant (1991) found no difference in NK activity between caregivers and controls. Esterling, Kiecolt-Glaser, Bodnar \& Glaser (1994) compared AD caregivers, former AD 
caregivers ( that is those whose relative had died at least two years previously) and controls. Similarities were found between the former and current caregivers in that they had poorer NK-cell response to stimulatory chemicals than the control group. Overall, these data suggest that psychological and immunological consequences of chronic stressors may persist beyond the cessation of the actual stressor.

Kiecolt-Glaser, Dura, Speicher, Trask \& Glaser (1991) studied family members who had been providing care to patients with alzheimers disease and other dementias for an average of 5 years. Caregivers showed lower overall immune functioning than controls on 3 functional assays (conA, PHA, elevated EBVAb).

\subsection{Impact of Stressors on Infection and Illness}

The research literature suggests that the impact of chronic stressors and psycho-social factors on the sympathetic nervous system and endocrine function influences the immune system, thereby providing shared mechanisms that may impact on disease susceptibility and progression across a broad spectrum of disorders (Kiecolt-Glaser \& Glaser, 1995). Very little research on acute stress and traumatic stress is mentioned in the literature, therefore little is known or can be deduced on the extent of immunological changes, disease susceptibility and progression in this area.

Stress and infectious disease has been an area that has received much attention over the years. Infectious diseases result from the growth and action of micro-organisms or parasites in the body and may or may not be contagious. The standard research criteria for diagnosis of clinical infectious disease require both biological evidence of infection and manifestation of related symptomatology (Beare \& Reed, 1977; cited in Cohen \& Willamson, 1991). Measures of disease symptomatology can be classified into two categories, namely signs and symptoms. Signs are observable, such as lesions, rashes and swelling. Technological equipment like $\mathrm{X}$-rays can be used to detect signs. Trained clinicians are often used to identify observable signs like oral lesions in herpes simplex. Symptoms on the other hand are not observable but are reported by a patient, for example, headaches and stomach aches. Many studies use unverified self-reported symptom protocols as their only criterion for disease. Cohen \& Williamson (1991) suggest that these reports may reflect influences of stress on cognitive processes and self perceptions that are not 
associated with infectious disease, even though there may be reflections of underlying infectious pathology.

\subsubsection{The Influence of Stressors on Infection and Illness}

It is apparent from the literature that stressors are thought to influence the pathogenesis of physical disease by causing negative affective states such as anxiety and depression. These states in turn exert direct effects on biological processes or behavioural patterns that increase disease risk. Substances such as alcohol and drugs are known to have direct and indirect effects on immune functioning. Indirect effects include alterations in nutrition, liver functioning and stress reactions. Furthermore distressed individuals often have appetite disturbances that can affect nutrition, consequently impacting on cell-mediated immunity, phagocyte function, complement system integrity and mucosal immunity. Keller, Shiflett, Schleifer \& Barlett (1994) suggest that there is considerable evidence that chronic or acute medical disorders as well as many medications can have immunologic or endocrine consequences. Smoking, caffeine intake and sleep deprivation all have effects on endocrine and immune functions. Physical activity may have both immunologic and endocrine consequences.

In order for infection to occur an infectious agent has to be present. Stress might influence susceptibility to infectious disease through various routes as illustrated below by Cohen \& Williamson (1991),

a. Altering biologic susceptibility and hence predisposing persons exposed to a pathogen to infection.

b. Initiating or triggering a process that allows a pathogen that is already in the body (for example a latent virus) to reproduce.

c. Contributing to the maintenance of an ongoing pathogenic process.

The routes have a direct effect on immune functioning. Cohen \& Williamson (1991) have proposed a model which represents plausible pathways linking stress to infectious pathology. Figure 2 (illustrated on the next page) indicates that stress may influence immunity either through direct innervation of the CNS and immune system (nerves terminating in lymphoid organs) or through neuroendocrine-immune pathways where there is a release of hormones. 
With regard to the hormonal pathway, a wide range of hormones released under stress have been implicated in immune modulation, such a catecholamines, epinephrine and norepinephrine secreted by the adrenal medulla, cortisol secreted by the adrenal cortex, growth hormone and prolactin secreted by the pituitary gland and the natural opiates beta endorphin and enkephalin released in the brain (Rabin, 1999)

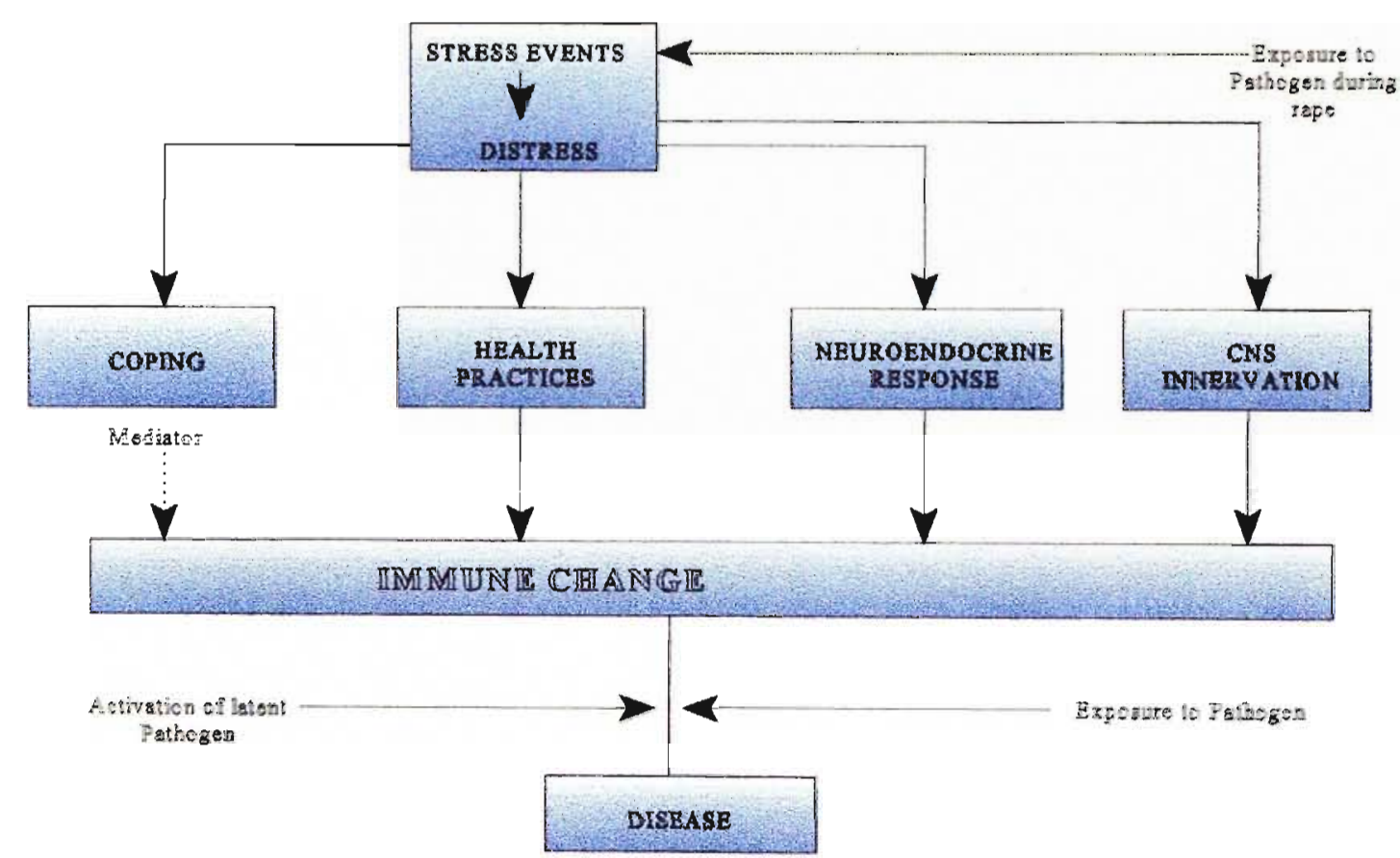

Figure 2: Behavioural and Biological pathways linking stress to the onset of infectious disease. In the case of rape, exposure to the pathogen can occur at the same time as the stressful encounter, (adapted from Cohen \& Williamson, 1991). 


\subsubsection{Stress and the Severity and Course of Infectious Disease}

The pathway illustrated in Figure 2 is also involved in shaping stress effects on duration and severity of disease. Cohen \& Williamson (1991) state that the course of illness may be influenced by direct effects on disease involved tissues which does not involve the immune system (for example, hormones such as cortisol and epinephrine triggered by stress may increase mucous secretion and vasodilation or control reflex responses which enhance symptoms such as irritation or sneezing). Changes in health practices may result in stress having an influence on disease involved tissues (for example, increased smoking under stress could irritate nasal and lung tissues, this has an indirect effect on the immune system). In addition, failure to comply with medical regimens under stress could result in more severe and longer lasting illness due to either undesirable behaviours which aggravate existing problems or because failure to perform desirable behaviours can result in disease progression. Cohen \& Williamson (1991) suggest that these actions may occur through influences on immune function or through influences of disease involved tissue.

Latent pathogens may be reactivated by stress. These latent viruses include oral and genital herpes and AIDS. Kiecolt-Glaser \& Glaser (1987) have found that reactivation can occur through hormonal or neural stimulation of pathogen reproduction or through suppression of aspects of the immune system that might otherwise hold the pathogen in check.

Other factors influencing susceptibility to infection include prior exposure to a pathogen and consequent development of immunity, characterised by the production of antibodies. The presence of antibodies is also an indication of prior exposure. Other factors which can influence risk of infection are nutritional status, previous history of illness, presence of other disease, geneticimmune factors, age, race, gender, pregnancy, rhythms (circadian, menstrual phase, annual) and seasons of the year (temperature and exposure to light).

The literature in psychoneuroimmunology pays much attention to the study of upper respiratory infections. It is worth reviewing some of the literature in this field as this type of infection occurs 
very frequently

\subsubsection{Upper Respiratory Infection}

The anatomical structure of the respiratory system makes it vulnerable to a wide range of viral and bacterial infections. Viral infections like the common cold and influenza are most familiar. There are more than 100 viruses that can cause colds whereas influenza is primarily caused by two types of viruses (A \& B). Colds and flu are both characterized by sore throat, congestion and mucus secretion, in addition to the symptoms already mentioned, flu can be accompanied by elevated temperature, gastrointestinal discomfort and joint pain. Viral infections of the upper respiratory tract are sometimes complicated by secondary bacterial infections caused by inflammation of mucous membranes reducing the ability to protect against pathogenic bacteria. A retrospective study conducted by McClelland, Alexander \& Marks, 1982), indicated that high numbers of stresses (for example upsets, worries and sources of tension), have also been associated with retrospective reports of upper respiratory infection severity as well as severity of all illness. It seems apparent that stress may also interact with other psychological variables in predicting upper respiratory infection. For example Sarason, Sarason, Potter \& Antoni (1985) found that those with many negative life events and few social supports were more likely to report chronic (mostly upper respiratory infections) illnesses than other groups.

McClelland et al. (1982) found that people who were both high in number of stresses and in need for power reported more severe illness than all other groups combined. Stone, Reed \& Neale (1987), found that stress may precede upper respiratory infection symptomatology by three to four days closer in time to incubation period of many common cold viruses which is 24 to 72 hours and other studies indicate stress-induced changes in immunity as well as illness behaviour (Glaser et al., 1982, cited in Stone, Valdimarsdottir, Jandorf \& Neale, 1987).

\subsubsection{Herpes Virus Infections}

Herpes simplex virus- 1 (HSV-1) is most frequently associated with cold sores, and HSV-2 is associated with genital lesions. Herpes viruses can cause a range of illnesses, for example generalised infections and encephalitis. Unlike other common viruses such as rubella that are usually eliminated by the immune response after a period of time, individuals remain latently 
infected for life after infection with any of the herpes viruses. When cellular immunity is compromised (for example people with Acquired Immuno Deficiency Syndrome (AIDS)), immunological control over herpes virus latency is impaired. In some cases reactivation of the latent virus may occur and may result in disease, (Kiecolt-Glaser \& Glaser, 1995).

Herpes virus disease recurrence varies and is thought to be influenced by fever, exposure to the sun, hormones and psychological factors such as stress, (Laudenslager, Van der Plate \& Aral, 1987, cited in Cohen \& Williamson, 1991). Kemeny, Cohen, Zegans \& Conant (1989) investigated the effects of various types of HSV reactivation in otherwise healthy subjects over a 6 month period outbreak of cold sores. Immune measures included enumeration of $\mathrm{CD} 4+$ and CD8+ cells and stressors and a distress measure (depression) were identified. The results revealed that middle to high chronic stress ( "residual stress," "anticipated life stress") were strongly negatively correlated with CD4 but not CD8 measures. Low level chronic stress (" daily hassles") was strongly negatively correlated with CD8 but not CD4. The overall stress (sum of all stress measures, averaged over 6 months) showed a strong negative correlation with both CD4 and CD8 ratios (averaged over 6 months). All distress measures (depression, anxiety, hostility) showed negative relationships to CD8 (but not all CD4) cells. Averaged overall stress was not related to herpes recurrence rate.

\subsubsection{Methodological, Conceptual and Statistical Issues in PNI Research}

Human studies in psychoneuroimmunology are faced with limitations as indicated below. In PNI research there is a preferential use of non-invasive measures. Studies are restricted to specific body compartments such as peripheral blood and skin, the spleen, the lymph nodes and the thymus are excluded (Schleifer \& Keller,1991). PNI studies use immunologic techniques that are relatively non-invasive, amenable to repeated measure and do not require specific sensitization. These constraints severely restrict our ability to fully assess PNI processes. Other methodological issues include day to day variations and the elapsed time between psycho-social measures and immune or health measures. Schleifer, Keller, Bond, Cohen \& Stein (1989) have shown that as much as $85 \%$ of the between group differences in laboratory assays are attributable to the day on which a sample was obtained and analysed. Diurnal variations in immunological parameters are well documented (Rabin, 1999). 
A sensible interval between the psycho-social and immune / health measures must exist, or else detection of relationships may be attenuated. A sensible interval (for example, assays obtained at a fixed time at the end of a study) will reflect to a variable degree actual relationships between mood and infection which may occur far enough in advance of an immune assay to permit any biochemical effects (generally a half-hour or more).

A measure at a single point in time may therefore be sufficient to assess the long-term impact of relatively stable characteristic, such as some coping styles and personality characteristics, however short-term or notably unstable variable, such as stressful life events or mood, would require repeated measures along with repeated immune and health assessments.

A literature search revealed that rape survivors as a population group has not as yet been considered in PNI research. Therefore research using rape as a example of traumatic stress is pioneering work that would undoubtedly contribute to this exploding field.

\subsection{Conclusion}

Kiecolt-Glaser \& Glaser (1995) state that individuals who are presumably most likely to show health changes in response to stressors are those whose immune system function is already compromised to some degree, either by an immunosuppressive disease like AIDS, or by a natural process such as aging that is associated with immunological down-regulation. Weaker immunological defences at the onset of a stressor could place these individuals at great risk, since smaller stress associated immunological decrements could have more important consequences. Linking to this is evidence that suggests that chronically distressed individuals, because of longer term immunological changes may also be at greater risk (Baum, Cohen, \& Hall ,1993). Research looking at the effect of stress on naturalistic stressors like rape has not been documented. Research of this nature would provide insight into the effect of stress on the neurological, endocrine and immune systems. 


\section{COPING}

\subsection{Introduction}

Coping refers to an individual's efforts to master demands which include conditions of harm, threat, or challenge that are appraised or perceived as exceeding or taxing his/her resources. (Lazarus \& Folkman, 1984). Appraisal refers to the evaluative process that imbues a situational encounter with meaning for the person. There are two kinds of cognitive appraisals namely primary and secondary (Fawzy \& Fawzy, 1995). Primary appraisal refers to our sense that something of importance is jeopardised or at stake, where as secondary appraisal refers to our evaluation of the ways of opposing demands and options. Constraints and resources moderate this sense of jeopardy. Appraisals can be separated into those that are concerned with the recognition that the individual is in jeopardy (appraisal of what is at stake) and those that are concerned primarily with the evaluation of resources and options available for managing potential or actual harm (appraisal of coping). This distinction is valuable in drawing attention to different sets of variables that interact in determining stress responses, coping patterns and adaptational outcomes.

There are three categories of stressful appraisals, namely appraisals of threat and harm-loss and challenge. Appraisals of threat and harm/loss are distinguished primarily by their time perspective, with threat referring to the anticipation of imminent harm and harm/loss referring to the judgement that damage has already occurred. Challenge on the other hand involves not only the judgement that a transaction contains the potential for harm and the potential for mastery or gain but also the judgement that this outcome can be influenced by the individual. Thus, in challenge, appraisals of stake and a sense of positive control are fused.

\subsection{Coping and adaptation to trauma and loss}

Traumatic stress is defined as events that involve serious threat to life or physical integrity, either of oneself or significant others (for example the destruction of a home or community that evokes feelings of extreme terror and helplessness), (APA, 1994). Traumatic events have also been described as those that shatter peoples' beliefs that they live in a meaningful, predictable world (Parkinson, 1997). Traumatic situations seem particularly important for psychological study because they have the power to transform individual and family lives, almost always drain personal 
resources, and have potential impact on well-being and adjustment ( Mikulincer \& Florian, 1996). Trauma includes incidents like major accidents, and violent crimes such as rape and incest. Aldwin (1994) states that it is only those accidents or crimes that involve severe injury and perceived threat to life which should be classified as trauma.

The perception of a threat activates the hypothalamus, which stimulates the pituitary gland to release hormones that activate the adrenal glands (Aldwin, 1994). Various efforts have been made to classify coping processes. The present study gives consideration to the taxonomy of coping designed by Lazarus \& Folkman ( 1984). These authors emphasize two major categories of coping which are problem-focused and emotion-focused modes.

Problem-focused coping refers to efforts to improve the troubled person-environment relationship by changing things (for example, seeking information about what to do, by holding back from impulsive and premature actions and by confronting the person or persons responsible for one's difficulty). On the other hand, emotional-focused coping refers to thoughts or actions whose goal is to relieve the emotional impact of stress (bodily / psychological disturbances). Emotionalfocused approaches are mainly palliative in the sense that such strategies of coping do not actually alter the threatening or damaging conditions but make the person feel better. Examples of this include avoiding thinking about the trouble, denying that anything is wrong, distancing or detaching oneself as in joking about what makes one distressed, taking tranquillizers or attempting to relax.

It should be noted that the above classification does not imply that either one type of coping is used exclusively. Rather complex combinations of problem-focused and emotion-focused methods are used to cope with stress. Monat \& Lazarus (1991) suggest that the coping methods depend upon the conditions being faced, the options available to us and our personality.

Esterling, Antoni, Kumar \& Schneiderman, (1990) found that the emotional expression and interpersonal coping style evidenced by healthy people dealing with stressful traumatic experiences were related to their Epstein Barr Virus antibody titres. More specifically subjects who abstained from disclosing emotional material on a laboratory writing task had elevated EBV 
antibody titres.

There is data suggesting that the impact of acute stressors may be attenuated by cognitive avoidance (Suls \& Fletcher, 1985). Higher life event stress before and during the study together with low levels of avoidant psychological coping were associated with an increased probability of an infectious illness. Avoidant psychological coping style appeared to protect against high life event stress (Turner-Cobb \& Steptoe, 1996). Turner-Cobb \& Steptoe (1996) also found that high levels of social support were not protective under high life event stress conditions. However when life event stress was low, high social support was associated with a reduced incidence of infectious illness. These authors also found that episodes of upper respiratory tract infectious illness were high.

\subsection{Coping Behaviour of the Rape Survivor}

In a traumatic rape experience there are three different phases that elicit different types of coping strategies (Aldwin, 1994). The first phase, regarded as the threat phase, is characterised by strategies like cognitive assessment concerning the nature and severity of the threat, verbal tactics and physical action.

The second phase refers to the attack itself. This phase involves both problem-focused and emotion-focused coping mechanisms. Problem-focused coping included physical action (fight or flight), as well as cognitive and verbal strategies. Emotion focused coping strategies includes affective responses like crying, screaming; psychological defences - mainly emotional numbing and dissicuaition and physiological reactions such as vomiting, passing out and urinating. If these strategies were used purposefully to deter the attacker, they could be regarded as being problemfocused coping strategies.

The third phase follows immediately after the attack. Strategies most commonly used are bargaining for freedom, freeing oneself and alerting others which might be seeking social support or reporting the rape to the relevant authorities. 


\subsection{Coping and Control}

The importance of peoples' perceptions of, and attempts to achieve control are especially salient when considering coping with negative life events like rape. Snyder \& Ford (1987) state that the appraisals of control may be an integral part of perceiving events as negative, conversely, the experience of negative life events can represent an inherent threat to an individual's sense of control. In considering coping, by definition, attempts to control or master the painful consequences of negative occurrences are examined. Coping behaviours can in fact represent specific attempts to restore a lost or threatened sense of control and mastery.

\subsection{Possible links between Stress, Coping \& IIIness}

There are three main ways in which stress might lead to somatic illness ( Holroyd \& Lazarus, 1982).

1. The disruption of tissue function through neuro-hormonal influences under stress. This means that under stress there are major outpourings of powerful hormones creating dramatic alterations in bodily processes such as pounding heart, sweating, trembling and fatigue.

2. Engaging in coping activities that are damaging to health for example poor diet, heavy use of tobacco and alcohol. Intrinsically noxious styles of living can increase the likelihood of disease by damaging the tissues of the body.

3. Psychological and / or sociological factors which consistently lead the person to minimise the significance of various symptoms or poor compliance with treatment programs.

While more research is certainly required before definitive conclusions can be drawn about the relationships between stress, coping and illness, theoretical and empirical advances have been progressing rapidly. 


\section{RAPE - A LIFE CHANGE EVENT}

\subsection{Introduction}

Rape is a traumatic incident that can bring about dramatic changes in a person's life. Rahe (1995) states that the change in a person's psychological equilibrium as a result of an event is the essential quality in that event's stressfulness. Life change events can exert predisposing as well as precipitating influences on a person's illness susceptibility. Pre-disposing life events include significant events, generally occurring early in life, that appear to mould a person's responses to similar events should they occur later in life. Precipitating life events are those life changes that take place within a year or two of the onset of an illness. A person's assessment of the degree of threat imposed by recent life events largely determine the person's emotional and psychophysiological reactions to those events. The subject's varying perceptions of threat also influence their subsequent selections of coping tactics (Lazarus \& Folkman, 1984).

A stressor is a demand that places an adaptational requirement on the organism. This leads to a state of stress, strain or distress that in turn, depend on external moderators such as social support and on internal moderators such as coping ability. The result can be no change, psycho-social growth or an adverse health change (Solomon, 1995).

Psychological distress can lead to adverse immunological changes providing one physiological pathway through which major and minor life changes might result in an increased incidence of infections and malignant disease. Glaser \& Kiecolt-Glaser (1994) claim that most individuals who experience major life changes either do not become ill, or they only experience short illness episodes. Actual organically based episodes of illness are a function of differential exposure to an infectious agent (example a virus). The probability of clinical illness and the intensity and duration of the illness are in part the product of the prior status of the individual's immune system. Following this line of reasoning, individuals who are presumably most likely to show health changes in response to stressors are those whose immune function is already compromised to some extent, particularly the elderly or people infected with the Human Immunodeficiency Virus (HIV) 
Turner-Cobb \& Steptoe (1996) suggested that the nature and timing of adverse life experiences is relevant. The accumulation of life events stress may make the individual more vulnerable to infectious illness for several weeks or months into the future (Cohen, Tyrrell \& Smith, 1993). Adults who experienced high levels of major life events and daily event stress over a six month period suffered more episodes of illness and longer symptomatic periods than did low stress groups, Graham et al., (1986, cited in Turner-Cobb \& Steptoe ,1996). Meyer \& Haggerty (1962, cited in Turner-Cobb \& Steptoe, 1996) observed that stressful daily events were more likely to precede than follow episodes of streptococcal infection. Adler \& Matthews (1994) have found that an increase in undesirable life events three to four days before symptom onset has been reported.

Turner-Cobb \& Steptoe (1996) compared participants who succumbed to verified respiratory infection and those who remained healthy. They found that risk was positively associated with life event stress experienced both in the twelve months before the study, and during the study itself, but that these effects were modulated by psychological coping style and social support. They also found that alcohol consumption is inversely associated with risk of upper respiratory tract infectious illness. These authors also found that episodes of upper respiratory tract infectious illness were preceded by high levels of perceived stress, but were also associated with a decrease in the number of daily hassles reported.

Kemeny et al., (1989) conducted an investigation of stressful life experience, negative mood, helper-inducer (CD4+) and suppressor-cytotoxic (CD8+) T cells and genital herpes simplex virus (HSV) recurrence rate, on individuals with recurrent herpes simplex virus. They found that subjects with high levels of stressful life experience over the study period (including current acute and on-going stressors, and anticipation of future stressors) tended to have a low percentage of CD4+ and CD8+ cells. Subjects with high levels of negative moods (anxiety, depression or hostility) tended to have low levels of CD8+ cells only. Depresive mood - HSV recurrence relationship was not dependent on changes in health behaviors such as alcohol consumption, sleep or exercise. It is suggested that chronic levels of depressive affect may result in decreased levels of CD8+ cells or an associated immunological parameter which may create a biological environment conducive to herpes simplex viral replication and lesion formation. 
Pike, Smith, Hauger, Nicassio, Patterson, McClintick Costlow \& Irwin (1997) examined the effects of antecedent chronic life stress on psychological and physiological responsitivity after acute challenge with a psychological stressor. The results revealed that acute psychological stress induced subjective distess; increases of circulating concentrations of epinephrine, norepinephrine, B-endorphin, adrenocorticotropic hormone (ACTH) and cortisol and a selective redistribution of natural killer $(\mathrm{NK})$ cells into the peripheral blood as compared with the controlled condition. Pike et al., (1997) concluded that when people who are undergoing chronic life stress are confronted with an acute psychological challenge, an exaggerated psychologic and peak sympathomedullary reactivity occurs that is associated with decrements in individual NK cell function and is protracted beyond termination of the stressor and sympathomedullary recovery.

\subsection{Definition of Rape}

Rape is an act of violence and humiliation that happens to be expressed through sexual means. Rape is an avenue for the expression of power and anger. Rape is an act of sexual intercourse with a female, against her will and consent, whether her will is overcome by force or fear resulting from the threat of force or by drugs or intoxicants, or when because of mental deficiency she is incapable of exercising rational judgement, or when she is below an arbitrary age of consent. Rape can occur between married persons and same sex persons. The crime of rape requires only slight penile penetration of the victim's vulva. Full erection and ejaculation are not necessary (Sadock, 1995).

\subsection{The Rape Survivor}

A women being raped is frequently in a life threatening situation. Shock and fright approaching panic are experienced during the rape, during which her primary motivation is to stay alive. The victim may try to defend herself by either screaming, trying to attack the rapist, trying to reason or plea with the rapist, or trying to establish some human contact with the rapist in the hope that he might reconsider the attack. The survivor might be forced to submit to the act, especially if physical violence or weapons (for example guns or knives) are used. 
Physical and psychological trauma is experienced by the victim. The survivor can be haunted or intimidated by various rape myths, for example - 'the manner in which she dressed asked for it .' (Rape Crisis, 1998; 1999).

\subsection{Factors Influencing the Impact of the Rape}

- The relationship with the rapist. The rapist could either be a stranger, friend, boyfriend, husband or member of the family. The type of person the rapist is, as well as his behaviour to the survivor during and after the rape also influences the victim's reactions (Lewis, 1994).

- The women's age and life experience

- The survivor's perceptions of the rape, the myths or beliefs she holds about rape (for example, I was drunk so I am to blame for what has happened to me) tend to have an influence on her reactions to the rape and how she deals with it. Lewis (1994) adds that the type of person the victim is, the nature of her life before the rape, her behaviour during and after the rape determine her reactions to the rape.

- The religion, class, culture and race of the victim in terms of beliefs and value system can influence the survivors reaction to the rape.

- $\quad$ The nature of the rape (for example, if it is associated with physical violence) can result in physical injuries, illnesses or disabilities as a consequence of the rape or if it was a gang rape. At present transmission of sexually transmitted diseases in particular Human Immunodefiency Virus poses an incredible threat to rape survivors (Pillay,1999). This is due to the direct unprotected physical contact and exchange of bodily fluids, like semen and blood.

- The presence or absence of social support and the reaction and treatment received from family, friends and other significant people in the rape survivors life.

- The interaction and treatment received from members of the medical and legal systems, if she should choose to report the case.

The act of rape is undoubtedly extremely traumatic for the victim and like other traumatic events it is sudden and unexpected, uncontrollable and out of the ordinary (Tedeschi \& Calhoun, 1995). Many traumatic experiences result in symptoms collectively classified under Post-traumatic Stress 
Disorder (PTSD). Rape survivors experience many of the symptoms of PTSD, after the rape, But PTSD does not sufficiently describe the symptoms rape victims might experience, since rape is an extreme violation and a life threatening attack (Lewis,1994). An umbrella

term, Rape Trauma Syndrome, has been coined to refer to the many, varied negative effects of rape.

\subsection{Rape Trauma Syndrome}

Rape trauma syndrome describes a pattern of physical, emotional and behavioural responses which occur as a reaction to a crisis (Rape Crisis Manual, 1998; 1999; McFarlane, 1995). Victims of rape respond to the trauma of rape in various ways (Lewis, 1994) therefore the symptoms of rape trauma syndrome may manifest themselves in varying degrees (Donaldson,1997). A woman is said to be experiencing rape trauma syndrome if many of the symptoms are experienced intensely and at the same time (Lewis, 1994), whereas Burgess \& Holmstorm, (1974, cited in Allison \& Wrightsman ,1993) report that all rape victims suffer from rape trauma syndrome to some degree. Donaldson (1997) asserts that even those who avoided a completed rape may experience symptoms of rape trauma syndrome.

The impact of rape on the immune system has not been researched to date, therefore it is worth reviewing the specific symptoms of rape trauma syndrome so that information can be gleaned about the connection between rape and the immune system.

Rape trauma syndrome may be divided into two phases, but each can adversely affect all facets of the victim's life, this includes physical, psychological, social and sexual aspects.

Phase one is characterised by the immediate reactions to the rape, which may last for days or weeks and are usually quite severe. The second phase looks at the long-term processes of coming to terms with the reactions and trying to deal with the pain and hurt in an effective manner. For the purpose of this research only phase one will be reviewed because the focus of this study is on the relationship between acute stress reaction of the rape and immunity. 
Phase one is regarded as the acute crisis phase or the impact phase which occurs immediately after the rape. Survivors of rape are in a state of shock, and can feel anxious and fearful. The fear can become so salient and demanding that it over powers the lives of its victims (Allison \& Wrightsman, 1993). This impact can produce symptoms of a physical, behavioural, emotional and cognitive nature.

\subsubsection{Physical Symptoms}

Shock may be expressed in various forms, from being absolutely calm and unemotional to shaking, crying or laughing hysterically, twitching an inability to think, feeling cold, faint, disorientated, nausea and vomiting. Colhoun \& Atkeson (1991) suggest that these reactions are common particularly when the event is sudden and unexpected.

Various illnesses and body pains are experienced by the rape survivor. Gynaecological problems include irregular, heavy periods, vaginal discharges, bladder infections, sexually transmitted diseases and pregnancy. The rape survivor may experience headache, stomach pain or pains in other parts of the body. There might be tears in the vagina or rectum, bruises or grazes. Cuts, bruises and lacerations might also be found on other body regions as well. The rape survivor might feel sick or dizzy or might experience irritation or soreness of the throat due to forced oral sex. These symptoms might be as a result of the shock of the rape, or a medical problem.

Sleep and eating disturbances might occur where the individual experiences difficulty falling asleep, waking during the night, being woken by nightmares or needing to sleep more than usual. Eating disturbances might be characterised by an increase or a decrease in appetite. There might be an increase or a decrease in energy levels.

\subsubsection{Behavioural Symptoms}

The rape survivor might cry often, have difficulty in concentrating or feel restless, agitated or lethargic. Fear can appear to be beyond the survivors control, and has been known to paralyse a survivor after being raped (Rape Crisis, 1998; 1999). The survivor may find it impossible to be present in a place or situation which resembles that of the rape. The survivor may fear different environments, being alone or in crowds of people, or the rape survivor may have sexual fears. 
The rape survivor may have problems with speech ranging from excessive speech to not speaking at all, in addition the rape survivor may stutter or stammer more than usual. The survivor may feel a loss of control and might try to hang on to what control she/he can after the rape by trying to continue ' living as normal' and repressing the incident. Reminders and recollections of the incident may result in feelings of anger and vulnerability.

The rape may convey to the survivor that she is not worthy of respect. Many survivors feel physically dirty and spend a lot of time washing over and over again to try and feel clean after the rape. The survivor might want to clean her home if she was raped there. The cleaning may appear to be out of control.

The survivor might display little interest or attention in herself or other people. She might feel worthless and stop caring for herself and other people close to her. The survivor may not want to socialize or alternately she may want to socialize more than usual. According to Tedeschi \& Calhoun (1995) withdrawal from others could result from a perception that others cannot understand them or help them in any significant way. The relationships with family, friends, a lover or a spouse could become problematic. The survivor might be irritable with whom she/he was close to prior to the rape. She may become very dependent on others or overly independent. A survivor may feel unable to be touched by anyone else or unable to touch any one else. A survivor might become promiscuous after the rape, because she feels worthless and useless and shows disregard for her body. There might be a loss of interest in previously interesting activities.

Changes in life style (for example, changes in jobs, moving house or avoiding work) might also occur. The survivor might start using legal or illegal substances after the rape, or she may increase her usage of alcohol, cigarettes or drugs, if she used substances before the rape. Tedeschi \& Calhoun, (1995) suggest that increased drug use is likely when the difficulties created by the trauma are long lived.

\subsubsection{Cognitive Symptoms}

Thoughts, images and reflections of the event often occur. Thoughts may also intrude indirectly. The individual may become a bit more vigilant, particularly in matters related to the trauma. 
Being exposed to highly negative events may decrease self-esteem, at least in the first days or weeks after the trauma (Kilpatrick, Veronen \& Best 1985). Perhaps the most significant way in which thoughts are affected is the struggle to achieve an understanding of what has occurred (Hodgkinson \& Stewart, 1991). For many people however, the struggle to understand why bad things have happened is a painful and difficult journey that may never be completely finished.

\subsubsection{Emotional Symptoms}

The specific types of emotions that predominate will vary among individuals and across specific circumstances (Tedeschi \& Calhoun, 1995). One emotion that many individuals experience is guilt (Van der Wal,1990). There is a feeling of remorse about what was done and what was left undone. This may reflect a belief that certain actions may have prevented the event. Anger and irritability are often felt by individuals dealing with major life problems (McCann, Sakheim \& Abrahamson, 1988). The anger may be expressed at various targets and the irritability may come and go. The anger experienced may be morally justified, such as anger felt by a rape survivor towards her assailant. One of the most frequently encountered emotional responses following major life trauma is fear and anxiety (McCann et al., 1988). When the circumstances to which individuals are exposed involve significant threat to life, health or to important property, it is likely that those individuals will feel apprehension, worry and concern for some time following the event. Anxiety is an emotional response most frequently reported by people who are faced with difficult circumstances in life. Another general emotional state that is quite common in people dealing with difficult life circumstances is depression. Although anxiety is more likely to occur when trauma involves major threat, depression is more likely when the circumstances involve significant loss (Tedeschi \& Calhoun, 1995).

Individuals exposed to highly stressful events are very likely to experience a constellation of distressing emotions. Although the specific patterns will vary from person to person, it is acceptable to say that unpleasant emotional states are almost certain to occur. In addition to the occurrence of these emotions, some changes in actions and behaviour may also occur. 


\section{METHODOLOGY}

\subsection{Rationale and Aim}

Rape is a traumatic event which has devastating consequences for the victim. These consequences can either be psychological or physical. Irrespective of the consequence, the rape would be experienced as a traumatic event and the victim would try to cope with it in order to maintain equilibrium. Stressful life events are commonly believed to alter immunity and hence susceptibility to immune system-mediated disease (Cohen \& Herbert, 1996).Recent research is psychoneuroimmunology has described rapid alterations in immune parameters in response to acute psychological stressors, hence research of this nature has significant implications for rape victims, who are at risk for contracting various infectious diseases.

According to Cohen \& Williamson (1991), examining the effects of single stressful events on immune function, especially more serious and even traumatic events would significantly strengthen the literature. Impact of severe events on immune system functioning would provide the fairest test of a stress-disease reaction. A literature scan revealed that no research within the psychoneuroimmunology arena, to date, has considered rape as an example of a traumatic event. Most have concentrated on stressful daily events, examination stress and laboratory stress. The present study would also reveal information about the nature of the stressor that puts a person at risk for disease, the timing of the stressor relative to exposure and disease onset as well as psychological and biological characteristics that moderate these effects.

This research aims to investigate the relationship between rape, coping style, the rape survivors' health profile and the immune system. Information gleaned from the present study is expected to have the following benefits :

a. Clinical care

Victims of rape are at risk of contracting various diseases and developing psychological problems, therefore research of this nature would aid in understanding and developing appropriate intervention strategies. This can be implemented soon after the incident, thereby preventing many adverse effects of the rape. 
b. Public health

If appropriate interventions are implemented based on the results obtained from this research, the transmission of various sexually transmitted diseases such as HIV infection might be curtailed, for example providing prophylactic treatment. Furthermore, psychological interventions implemented after the incident would be beneficial with regard to the maintenance of good mental health.

c. Financial

Interventions aimed at preventing negative consequences, soon after the rape could save the health care services large amounts of money that is being spent on medical (for example, the treatment of sexually transmitted disease) and mental health care.

d. Health Care Policies

Information gleaned from this research would also provide relevant information to facilitate the development of health care policies (for example policies pertaining to the treatment made available to rape survivors following the rape) and also highlight the importance of immunity as a link between the mind and body.

\subsubsection{Hypotheses}

a) There is a relationship between levels of distress and the parameters of the immune system.

b) There is a relationship between ways of coping and the parameters of the immune system.

c) There is a relationship between the health profile and the parameters of the immune system.

\subsection{Subjects}

A total of 36 rape survivors were initially recruited from the South African Police - Family Violence, Child Protection and Sexual Offences Unit in Pietermaritzburg and The Mathma Ghandi Hospital in Durban. However data collected from a homogenous sample of 12 female rape survivors were included in the present research. Table 1 illustrates the sample size at time 
1, 2 and 3. The sample size was further reduced, because all subjects needed to be studied at the same time of day to minimize error variance associated with diurnal variation in both neuroendocrine and immune parameters (Ottaway \& Husband, 1992). The subjects were above 18 years of age, and both English and Zulu speaking. Rape victims with severe health impairment and those who were pregnant were not included in the research. The selected sample were not aware of the HIV status before the study. In the present study the participants would be referred as rape survivors and the work will refer to the more commonly affected gender as 'she/her' even though it is acknowledged that male are also exposed to rape.

There were difficulties in obtaining a larger sample because the rape survivors would arrive late due to transport problems. The majority of them lived in rural areas and relied on public transport. They often did not have money to pay for transport. Money was made available to them for this purpose, however it was not used appropriately. It was not possible to obtain data from people who arrived late as the blood samples had to be at the laboratory by 11 h00 the latest. This was because certain blood tests had to be carried out within 12 hours after the blood samples were collected. Contacting rape survivors was also problematic as many of them did not have access to telephones or easily accessible residential areas. These problems also contributed to the small number of rape survivors following through with their procedures through time 2 and time 3 .

Table 1: Sample size across time 1,2 and 3

\begin{tabular}{|l|l|l|l|}
\hline & Time 1 & Time 2 & Time 3 \\
\hline $\begin{array}{l}\text { Psychological } \\
\text { measures }\end{array}$ & $\mathrm{N}=36$ & $\mathrm{~N}=6$ & $\mathrm{~N}=5$ \\
\hline Blood Measures & $\mathrm{N}=12$ & $\mathrm{~N}=6$ & $\mathrm{~N}=5$ \\
\hline
\end{tabular}




\subsection{Measures and Instruments Used}

The psychological and immunological measures are outlined below.

- $\quad$ Stress - assessed using the Brief Symptom Inventory (BSI) (Derogatus \& Spencer, 1982).

- Impact of the Rape - assessed using the Revised Impact of Event Scale (Horowitz, Wilner, \& Alvarez, 1979).

- Coping - assessed using the Ways of Coping Checklist (WCC) (Folkman \& Lazarus, 1980).

- $\quad$ Recent Life Changes - assessed using the Recent Life Change Event Questionnaire (Rahe, 1995).

- Health Status - assessed using the Symptom Status Scale of the Duke-University of North Carolina Health Profile (Parkerson, Wagner, Clapp \& Muhlbaier, 1981).

- Immunological Measures - CD3, CD4, \& CD8 levels, HIV antibody test, Full Blood Count which includes neutrophils, monocytes, eosinophils, lymphocytes, white cell count and platelets. (Appendix 1 provides a summary of the functions of these cells).

\subsubsection{Brief Symptom Inventory (BSI)}

The BSI was developed as a result of experiences with the SCL-90-R which revealed that even though time demands (15 - 20 minutes) were relatively unimposing, there were situations which could not even afford such a brief assessment period. Evaluation of the Item-dimension correlations from the SCL-90-R (Derogatis \& Cleary, 1977a cited in Derogatus \& Spencer,1982) suggested that 5 to 6 items on each sub-scale were sufficiently saturated to sustain an operational definition of each syndrome construct. The highest loading items of each dimension were selected to form the Brief Symptom Inventory. The BSI has found greatest application in clinical and research situations.

The BSI is a 53 item self-report symptom inventory designed to reflect the psychological symptom patterns of psychiatric and medical patients as well as non-patient individuals. The BSI 
may be used in a single, point-in-time assessment of an individual's clinical status, or it may be utilized repeatedly, either to document trends through time, or in pre-post evaluations. For the purpose of the study the BSI was used to monitor trends through time.

Each item of the BSI is rated on a 5 point scale of distress $(0-4)$. This ranges from " not at all "(0) at one end to " extremely" (4) at the other end. The BSI is scored and profiled in terms of nine primary symptom dimensions. The nine dimensions are 1) somatization; 2) obsessivecompulsive; 3) interpersonal; 4) depression; 5) anxiety; 6) hostility; 7) phobic anxiety; 8) paranoid ideation and 9) psychoticism. Three global indices have been developed, namely:

1) Global Severity Index (GSI)

2) Positive Symptom Distress Index (PSDI)

3) Positive Symptom Total (PST)

Since this research is concerned with the level of distress, only the Global Severity Index (GSI) is utilized and will be discussed briefly.

The GSI was employed in this study because it is the most sensitive indicator of a respondent's distress level (Derogatis \& Spencer, 1982). This is because information of the intensity of distress and numbers of symptoms are combined. The GSI produces a score that is calculated by dividing the total score obtained on the BSI by the number of symptoms that were positively responded to. This means that the total score is divided by the number of symptoms that were scored more than 0 .

\section{Reliability}

The reliability co-efficient for the global distress index is 0.90 , indicating good test-retest reliability. Test-retest coefficients range from a low of 0.68 for somatization to a high of 0.91 for phobic anxiety. Therefore the BSI is a consistent measure across time. Internal consistency coefficients were established on a sample of $n=719$ psychiatric outpatients, using Cronbach's co-efficient (alpha). Alpha coefficients for all 9 dimensions of the BSI were very good, ranging from a low of 0.71 on the psychoticism dimension to a high of 0.85 on depression. The Cronbachs' alpha for the BSI scales are as follows : Somatization - 0.66; Interpersonal Sensitivity - 0.74; Anxiety - 0.63; Phobic Anxiety - 0.79; Obsessive Compulsive - 0.60; Depression - 0.91; Paranoid Ideation - 0.78; Psychotism - 0.76 . 


\section{Validity}

Predictive validity has been established with respect to various populations.

Even though this scale has revealed valid results (Derogatus \& Spencer, 1982), its applicability to the population researched (that is, mostly Zulu speaking South African rape victims) in this study is limited. This is because the instrument has not been standardised on a South African Zulu speaking population. Therefore, no attempt is made to use the established norms, but rather results obtained from this study are used comparatively within the sample. The level of distress in this study is examined in relation to demographic factors, coping behaviours, recent life changes, health status reported, and immune parameters.

\subsubsection{Revised Impact of Events Scale: A Measure of Subjective Distress}

The Impact of Events Scale (Horowitz, et al., 1979) is used to assess current subjective distress for any life event. The instrument was developed so that investigators could observe individuals over periods of time following the occurrence of an event; compare subgroups for degree of subjective distress after a particular life event; or contrast life events in terms of their relative impact on different populations.

The scale initially consisted of 20 items. Nine items described episodes of intrusion, and eleven items described episodes of avoidance. This was then revised to include 15 of the 20 items (Horowitz, et al., 1979). The 15 items are divided into 2 clusters. The primary cluster contained items from the clinically derived intrusion subset, while the second cluster was composed of clinically derived avoidance items. Only items that empirically clustered and those that had significant item-to-sub-scale correlations beyond the 0.01 level of significance were considered in the revised scale. Only the frequency variable was included, because it was scored more accurately by subjects than the intensity variable.

The mean total stress score $\quad=39.5(\mathrm{SD}=17.2$, range 0-69)

The mean intrusion sub-scale score $=21.4(\mathrm{SD}=9.6$, range $0-35)$

The mean avoidance sub-scale score $=18.2(\mathrm{Sd}=10.8$, range $0-38)$

The Intrusion sub-scale measures unbidden thoughts and images, troubled dreams, strong pangs, waves of feelings and repetitive behaviour. The avoidance sub-scale measures ideational 
constriction, denial of the meanings and consequences of the event, blunted sensation, behavioural inhibition or counter-phobic activity and awareness of emotional numbness.

\section{Reliability}

The split half reliability for the revised scale is $(r=0.86)$. Internal consistency of the sub-scale, which was calculated using Cronbach's alpha for avoidance was 0.82 and intrusion $=0.78$. The Cronbachs' alpha calculated for this study for avoidance was 0.27 and for intrusion was 0.78 . A correlation of $0.42(p>0.0002)$ between the intrusion and avoidance sub-scale scores, for the revised scale, indicated that the two subsets are associated, however they do not measure identical dimensions. Test-retest reliability of 0.87 for the total stress scores, 0.89 for the intrusion sub-scale and 0.79 for the avoidance sub-scale were obtained. The sensitivity of this scale has been supported, where change was indicated in populations by clinical impressions, by experienced observers or by relevant differences in the response to discrete life events of varied magnitude. Horowitz et al (1979) reported that persons of various educational, economic and cultural backgrounds have been able to use this scale.

\subsubsection{Ways of Coping Checklist}

The Ways of Coping Checklist (WCC) (Folkman \& Lazarus, 1980), is a self report measure comprising of 68 items which represent a wide range of coping strategies that a person may employ when attempting to deal with a stressful experience. The items of the WCC are classified into two broad categories, namely problem-focussed and emotion-focussed. The problemfocussed approach includes items that refer to cognitive problem-solving efforts and behavioural strategies that change or manage the source of the problem. The emotional focussed approach focuses on cognitive and behavioural efforts aimed at regulating or reducing emotional distress.

The WCC has been criticised for not defining more specific coping strategies used to deal with stressful situations (Eagle,1987). Vingerhoets \& Flohr (1984) were among those who criticized the WCC on these grounds. They therefore adapted the WCC by developing various coping subscales to assess more specific coping strategies. A principle component analysis with varimax rotation yielded six factors with eigen values above 2.0, (Eagle, 1987). The incorporation of these factor sub-scales resulted in a 60- item version of the WCC. Eagle (1987) found the 
problem focussed / help-seeking scale problematic, as it appeared to subsume two relatively distinct coping styles. The first focussed on coping strategies which involved direct cognitive or behavioural problem-solving efforts. The second focused on specific help seeking activities. Eagle (1987) addressed this by developing a 4-item scale focussing only on help-seeking efforts. This allowed direct comparison on the help-seeking variable alone.

\section{Reliability}

The reliabilities (Cronbach's alpha corrected for the number of items) of the scales used in the Eagle (1987) study were as follows:-

$\begin{array}{llll}\text { - } & \text { wishful thinking / escape } & - & 0.784 \\ \text { - } & \text { acceptance } & - & 0.647 \\ \text { - } & \text { problem focussed / help-seeking } & - & 0.392 \\ \text { - } & \text { emotional withholding } & - & 0.603 \\ \text { - } & \text { growth-blame } & - & 0.590 \\ \text { - } & \text { help-seeking } & - & 0.680 \\ & & - & 0.698\end{array}$

The Cronbach's alpha calculated for this study are as follows:-

$\begin{array}{llll}\text { - } & \text { wishful thinking / escape } & - & 0.691 \\ \text { - } & \text { acceptance } & - & 0.779 \\ \text { - } & \text { problem focussed / help-seeking } & - & 0.467 \\ \text { - } & \text { emotional withholding } & - & 0.744 \\ \text { - } & \text { growth } & - & 0.681 \\ \text { - } & \text { help-seeking } & - & 0.770 \\ & & - & 0.735\end{array}$

The version of the WCC used in this study is the same as that used by Eagle (1987). One of the items on this version was excluded due to difficulties in translation from English to Zulu. The categories of the revised version are briefly discussed below: 
- Wishful thinking-escape

This pertains to emotion-focused coping strategies, which involve cognitive efforts to escape from emotional discomfort, by techniques such as wishful thinking, denial and humour.

- Acceptance

This involves an acceptance of stress after it has emerged. It refers to emotion-focused coping strategies such as compromise, substitutive activity and patience.

The scale incorporates both cognitive and emotional strategies for minimizing the effect of stress. Several items included in this scale are negatively correlated with acceptance and are scored in a reverse direction.

- Problem-focused / help-seeking

This refers to problem-focused efforts which seek to change or act on the source of the problem, and emphasize problem-solving and direct action. It includes items which infer seeking advice, finding alternative solutions, and decisive behavioural planning.

- $\quad$ Self-Blame

This involves emotion-focused strategies that indicate an inclination to respond to stressful situations by criticizing or blaming oneself, for not being able to cope with these situations. Items included illustrate a wish to be a more assertive and 'strong' person, thus indicating a dissatisfaction with ones present coping abilities.

- Emotional withholding

This scale involves emotion-focused strategies that aim to control anxiety through the inhibition of emotional discomfort. This implies an unwillingness to look for or accept emotional support from others (independence), or to express feelings of vulnerability or dependence. Some items are negatively correlated with emotional withholding and are scored in a negative direction

- Growth

This pertains to emotion-focused strategies that attempt to buffer the impact of a stressful situation by controlling the meaning of the problem, thus serving to identify the creative and growth potential stressful situations may present. 


\section{- Help-seeking}

This refers to specific problem-focused strategies where efforts are directed towards others to obtain information and assistance.

\subsubsection{Recent Life Change Questionnaire}

The first version of the questionnaire, devised in 1963, was designed to measure the 43 life change events. This was called the Schedule of Recent Events ( SRE). In 1975 Rahe (cited in Rahe, 1995) expanded the SRE to 74 recent life change events and titled the new form the Recent Life Changes Questionnaire (RLCQ). Forty two of the original 43 life change events from the SRE were kept in the RLCQ, only Christmas was deleted. The life scaled units for the RLCQ published in 1978 were re-scaled in 1994. The rescaled version was used in this study.

Recent life changes were categorised as follows: health, work, home and family, personal and social, and financial. The values for life-change intensity can be summed over arbitrary defined intervals of time such as 0-6 months ; 7-12 months, 13-18 months and 19-24 months ago. Yearly life change estimates have been expressed as life-change units (LCU's). Low, intermediate, elevated and high LCU ranges have been established that provide quantitative estimates for the person's amount of recent life change ( Rahe,1995 cited in Kaplan \& Sadock,1995).

\subsubsection{Duke University of North Carolina Health Profile (DUHP)}

The DUHP is a health status measure for adults. This measure is designed for research and for day to day clinical assessment in the primary care setting. The DUHP measures health status along four dimensions including symptom status, physical function, emotional function and social function. The physical, emotional and social function dimensions have been shown to contribute independent information to the assessment of health (Ware, Brook, Williams et al. 1978, cited in Parkerson et al. 1981). Since this measure has importance in primary care, symptom status was conceptualized as a separate dimension despite its overlap with other dimensions. 


\section{Conceptualization of each of the four dimensions of the DUHP}

1. Symptom Status:

Symptoms are a natural expression of dysfunction within the body and mind. Symptoms are often the earliest and sometimes the only manifestation of altered health. The number and severity of symptoms provide an indicator of general health status, even though they might often be vague and nonspecific, where the same symptom can result from a variety of physical or psychological disorders. Symptoms are relevant to outcome measurement in the medical care setting, because most patients present with one or more symptoms that influence the selection of diagnostic studies and subsequent treatment (Parkerson, et al. 1984).

Symptom status can also be viewed as an indicator of overall general health, since physical, emotional and social problems frequently work independently or in combination to produce symptoms in the individual.

\section{Physical Function}

This dimension comprises three distinct components which are listed below:

a) disability days (confinement to home or bed)

*this is a traditional measure of a person's response to illness, whether it is a chronic disease, injury or self-limited respiratory infection

b) ambulation

*these items range from inability to walk to the bathroom to the capacity to run long distances. The running items reflect the emphasis of the DUHP on health rather than illness.

c) items relating to upper extremity function are included because of the frequency of hand and arm problems resulting from injuries.

The physical function scale measures an individual's perceived capacity to perform tasks rather than requiring a report of actual performance (for example a healthy person may be able to run a mile without difficulty but simply has not done so in recent weeks. This might be for reasons totally unrelated to health). 


\section{Emotional Function}

This is assessed by examining the respondents level of self-esteem. Self-esteem is defined generally as a liking and respect for oneself and the belief in one's ability to get along with other people (Coopersith, 1967). This author also suggests that self esteem is an indicator of emotional function based on the presumed importance of ego strength to emotional well-being. The authors of the DUPH claim that this approach accents health rather than mental illness and avoids diagnostic inventions for depression and anxiety.

\section{Social Function}

This is a measure of a person's ability to perform his/her usual role in society. Role performance is assessed in four areas as listed below:

1) self care

2) ability to function in the workplace or at home

3) interactions with people

4) participation in community and social events

These items are general, in order to accommodate the wide variety of activities that make up the life style of different individuals.

The DUHP consists of 74 items distributed among the 4 dimensions as follows:

1) symptom status - 28 items

2) physical function - 15 items

3) emotional function - 26 items

4) social function - 5 items

Scoring of the DUHP involves a simple linear scale with the response for the least desirable health status receiving a score of zero and those with the most desirable receiving scores of 2 for a three-point scale, 3 for a four-point scale and 4 for a five point scale. A separate score was determined for each of the four health dimensions by dividing an individual's score for each dimension by the maximum possible score for that dimension. The resulting four health dimension scores for each person were expressed as a proportion ranging from 0,00 for the worst possible to 1,00 for the best possible health status. Each item has an equal weight in the scoring within its 
respective health status dimension. Responses to all DUHP items, except those concerning disability days are considered to be indicative of health status regardless of the respondent's perception of the relationship to personal health.

\section{Critique of the DUHP}

The use of specific indicators like the symptom status, physical function, social function and emotional function might limit comprehensiveness. However overlap among the 4 health dimensions is reduced and clear conceptual definitions of the components being measured, are provided.

\section{Reliability}

Parkerson et al., 1981 suggests that fluctuating quality of health, along with individual variations in reporting of symptoms and functional status pose difficulties for reliability testing, however sufficient reliability has been established. Internal consistency for 23 items on the emotional function dimension was 0,85 as measured by Cronbach's alpha. Guttman scalagram analysis applied to the ambulation items in the physical function dimension revealed high co-efficients for reproducibility (0.98) and scalability (0.89). In the social function dimension scalagram analysis produced Guttman coefficients of 0,93 for reproducibility and 0.71 for scalability. Overall testretest coefficients for the four scales are as follows; overall physical function $=0.82$; emotional function $=0.72 ;$ symptom status $=0.68$ and social function $=0.52$. The current study yielded Cronbach's alpha of 0.885 for the symptom status dimension; 0.573 for the physical function dimension; 0.808 for the emotional function dimension and 0.740 for the social status dimension.

\section{Validity}

In the development of this scale, health is recognised as being too complex a state to be considered a single construct. Therefore, no overall score was developed and validity investigations were separate for each of the 4 health dimensions. 


\subsection{Translation of Instruments}

In this study self-report measures were used, with the exception of the blood measurements. Since the original measures are in English, it was necessary to have all the questionnaires translated into Zulu. The back-translation method was adopted for these measures. The translation process initially involved translating the questionnaires into Zulu by first language Zulu speakers. The measures were then translated into English by other first language Zulu speakers, who were fluent in English and had not seen the original English version of the questionnaires. The translation was then checked against the original. Corrections were then made to the Zulu translations and the measures were then rechecked against the original version. This was done to ensure that the original meaning of all the items was retained.

\section{$\underline{5.5} \quad$ Procedure}

The initial phase was to obtain ethical approval from the Ethics Committee, Faculty of Medicine, University of Natal. Once this was granted, the head of Forensics Medicine, the Superintendent of Mathma Ghandi hospital, the Superintendent of the Family Violence, Child Protection and Sexual Offences Unit - SAP and the head of the District Surgeons were consulted to obtain permission to collect data within the respective centres. Negotiations were conducted with the laboratory regarding the analysis of the blood.

The district surgeon conducted an examination to confirm whether or not the person had been raped. All rape survivors were handed a letter outlining the nature, the benefits and the limitations of the research. Informed consent was obtained from each participant, after the nature and possible consequences of the study was fully explained by the researcher. All participants received HIV/AIDS pre-test counselling prior to the data collection and post-test counselling once the results were obtained. The counselling was conducted in the participants home language by a trained nursing sister. The subjects were given verbal instructions regarding the completion of the questionnaire. It was stressed that their personal details were confidential and that they were free to decline or refrain from answering questions at any point of the research. Following the administration of the questionnaire, blood samples were drawn. The administration of questionnaires and drawing of the blood had to occur before $10 \mathrm{~h} 30$. This was because the blood samples had to be transported to the haematology laboratory by $11 \mathrm{~h} 30$, at the latest, for analysis. 
If the bloods were drawn in Pietermaritzburg, they were either transported by the Provincial Hospital transport or transported by a private courier service. If the bloods were drawn at Mathma Ghandi Hospital they were transported by the research assistant to the laboratory. All bloods were transported at room temperature. The bloods had to be analysed on the same day because of the short lifespan of the cells. The procedure for time 1 (within 5 days of the rape), time 2 ( 15 days after the rape) and time 3 ( 35 days after the rape) were similar. HIV testing was only carried out at time 1 . Money for transport was provided to those who could not afford it and to those who wanted to continue with the research procedure. Both HIV positive and HIV negative rape survivors underwent the same research procedure.

\subsection{Data Analysis}

Data obtained from the study was subjected to a series of statistical analyses using the SPSS package (Norusis, 1990). These are outlined below.

* Reliability tests were run on all the data. Method 1 (space saver for the reliability analysis) was used. The Cronbach's alpha obtained for the specific sub-scales have been included in the methodology.

- Descriptive statistics on the demographic factors measured by this study.

- The relationship between levels of distress, ways of coping, health profile and immune parameters was investigated by conducting Pearsons' Product Moment correlations. Scatterplots were used to supplement these findings.

- Finally Friedman Tests were conducted to investigate the difference in the mean scores across times 1,2 and 3 . 


\section{6. $\quad$ RESULTS}

This section initially looks at a brief outline of descriptive statistics. The descriptive statistics include the demographic details and an analysis across time of the distress of rape, the impact of the rape, the ways of coping and the health profile. The testing of the hypothesis include the relationship between the distress of rape, the impact of the rape ways of coping, health profile and various immune parameters. The small sample and many variables contributed to constraints in the method of data analysis used. The findings presented in this chapter aim to explore the relationship between rape survivors levels of distress, ways of coping, health profile and immune parameters across time. Several correlation matrices were obtained to investigate the above. However it is important to note that the great number of correlation coefficients derived from the many comparisons of variables in itself is a problem since a large pool are likely to arise purely by chance and it would be an impossible task to decide on which to accept and which to reject in order to counter this effect. Only significant results are presented in this section. The nonsignificant results are available from the author - School of Psychology, University of Natal, Pietermaritzburg, for perusal.

\subsection{Demographic Details}

The demographic details are illustrated in Tables $2 \mathrm{a}-2 \mathrm{~h}$.. The demographic details provide information about the profile of the rape survivors participating in the present study.

Table 2a: Participants by Language

\begin{tabular}{|l|l|}
\hline First Language & Percentage \\
\hline English & 8 \\
\hline Zulu & 92 \\
\hline
\end{tabular}

Table $2 \mathrm{~b}$ : Participants by Ethnic Group

\begin{tabular}{|l|l|}
\hline Ethnic Group & Percentage \\
\hline African & 92 \\
\hline Indian & 8 \\
\hline
\end{tabular}


Table 2c : Participants by Religious Affiliations

\begin{tabular}{|l|l|}
\hline Religious Affiliation & Percentage \\
\hline Christian & 91.7 \\
\hline Hindu & 8.3 \\
\hline
\end{tabular}

Table 2d: Participants by Age

\begin{tabular}{|l|l|}
\hline Age & Percentage \\
\hline $18-25$ & 25 \\
\hline $26-30$ & 41.7 \\
\hline $31-35$ & 16.7 \\
\hline $36-40$ & 8.3 \\
\hline $41-45$ & 8.3 \\
\hline
\end{tabular}

Table 2e: Participants by Marital Status

\begin{tabular}{|l|l|}
\hline Marital Status & Percentage \\
\hline Married & 16.7 \\
\hline Single & 83.3 \\
\hline
\end{tabular}

Table 2f: Participants by Current Employment Status

\begin{tabular}{|l|l|}
\hline Employment Status & Percentage \\
\hline Full time & 16.7 \\
\hline Part time & 16.7 \\
\hline Unemployed & 66.7 \\
\hline
\end{tabular}

Table $2 \mathrm{~g}$ : Participants by Level of Education

\begin{tabular}{|l|l|}
\hline Educational Level & Percentage \\
\hline Less than high school & 33.3 \\
\hline High school standard completed & 66.7 \\
\hline
\end{tabular}


Table 2h: Participants by HIV status

\begin{tabular}{|l|l|}
\hline HIV Status & Percentage \\
\hline Positive & 42 \\
\hline Negative & 58 \\
\hline
\end{tabular}

Tables $2 \mathrm{a}-2 \mathrm{~h}$ indicate that the sample was not evenly distributed across ethnic group, language preference, religious affiliation, marital status, employment status, level of education and HIV status. Rape survivors in this study are characterised as being young, non-white, predominantly Zulu speaking, single, unemployed women who have at least a high school standard of education completed.

\subsection{Analysis Across Time}

The following section looks at a descriptive analysis of the blood measures, levels of distress, impact of the rape, ways of coping and health profile across an acute stress period. The three measurements across this time period are time 1 ( 5 days after the rape); time 2 ( 15 days after the rape ) and time 3 ( 35 days after the rape ). The analysis is conducted on the participants individually as well as together.

\subsubsection{A summary of the psychological and immune measures for each participant across time (the data is presented in Appendices 6 and 7)}

\section{Participant 1}

Observation of the scale of the Brief Symptom Inventory reveals that participant 1 appears to be consistent in her responses across time 1,2 and 3. The Revised Impact of Events Scale indicates a decrease in the items reported on the Intrusion Scale from time 1 to time 2. The Intrusion Scale remains constant from time 2 to time 3. A different pattern is observed on the Avoidance Scale. There is a gradual elevation of items being reported on the Avoidance Scale across time. The Ways of Coping Scale appears to be fairly consistent across time. A decrease is noted on the Problem-focused / Help- Seeking Scale, the Growth Scale and the Help-Seeking Scale from time 1 to time 2. An increase is noted from time 1 to time 2 on the Acceptance Scale, the Emotional- 
withholding scale and the Somatization Scale. The Wishful thinking / Escape Scale was elevated the most at time 2. The Self-Blame Scale was constant across time. The Duke-UNC Health Profile revealed that this participant obtained the best possible health status on the Physical Function Scale and the Social Function Scale. However a different picture was observed on the Symptom Status Scale, there is a decrease between time 1 and time 2, and an elevation in this dimension of health status from time 2 to time 3. The Emotional Function Scale reveals a consistent level of health Status in this dimension between time 1 and time 2. However between time 2 and time 3 a decrease is noted. Observation of measures of the immune system reveal no dramatic changes in the individual measures across time.

\section{Participant 2}

Observation of the results indicate a slight variability in the responses across time 1, time 2 and time 3. Inspection of the scale of the Brief Symptom Inventory reveals a decrease in the symptoms reported on the Somatization Scale, the Depression Scale, the Hostility Scale and the Psychotism Scale. The Obsessive-Compulsive Scale is the only scale that decrease from time 1 to time 2, with a slight increase from time 2 to time 3 . The Interpersonal-sensitivity Scale, the Anxiety Scale and the Paranoid Ideation Scale reveal a decrease from time 1 to time 2. These scales remain constant across time 2 and time 3. The Phobic Anxiety Scale remains constant across time 1 and time 2 , this is followed by a slight decrease at time 3 . The Revised Impact of Events Scale indicates an increase across time on the Avoidance Scale. An increase from time 1 to time 2 is noted on the Intrusion Scale, this is followed by a decrease from time 2 to time 3 on this scale. There are no dramatic changes in the Ways of Coping across time. The Duke-UNC Health Profile produces variable trends in the responses across time. The Symptom Status Scale increase from time 1 to time 2 and this is followed by a decrease from time 2 to time 3 . The Physical Function Scale reveals an opposite pattern with a decrease from time 1 to time 2 and an increase from time 2 to time 3. Both the Emotional Function and the Social Function Scales decrease across time. Observation of the measures of the immune system reveals no dramatic change across time.

\section{Participant 3}

Participant 3 has elevated levels of distress at time 2 as measured by the Brief Symptom 
Inventory. This trend is also observed on the Intrusion Scale and the Avoidance Scale, which are sub-scales of the Revised Impact of Events Scale. The Ways of Coping employed by this participant is variable across time. There is a decrease in the use of Wishful thinking / Escape methods across time 1,2 and 3. The Emotional Withholding Scale on the other hand in creases from time 1 to time 2 and decreases from time 2 to time 3 . The opposite pattern is observed on the Acceptance Scale, Problem-focused / Help-Seeking, Self-Blame, Growth and Help-Seeking Scale, that is there is a decrease in the use of these coping skills from time 1 to time 2 and an increase from time 2 to time 3 . With regard to the Dike-UNC Health Profile, participant 3 appears to have noticeable changes in health status from time 1 to time 2 on all scales. However there are no dramatic changes noticed from time 2 to time 3 . This participant appears to be an outlier on some of the immune parameters. Observable changes are evident for the CD 3 Absolute levels across time 1,2 and 3 ; the CD 4 Absolute levels from time 2 to time 3 and an increase in Neutrophil \% and Monocyte Absolute from time 2 to time 3.

\section{Participant 4}

Observation of the psychological measures presented in tables 18 reveal variable patterns. The Brief Symptom Inventory reveals that the Somatization Scale, the Interpersonal-Sensitivity Scale and the Paranoid-Ideation Scale all decrease from time 1 to time 2 and increase from time 2 to time 3. The opposite, that is an increase from time 1 to time 2 and a decrease from time 2 to time 3 is observed on the Hostility Scale. The Depression Scale, the Obsessive-compulsive Scale and the Psychotism Scale all decrease across time 1, 2 and 3. The Anxiety Scale remains constant across time. Whereas the Phobic Anxiety Scale remains constant across time 1 and time 2 and decreases across time 2 and time 3. With regard to the Revised Impact of Events Scale both the Intrusion Scale and the Avoidance Scale increase from time 1 to time 2 and decrease from time 2 to time 3. Variable results are also evident for the Ways of Coping Checklist. The Growth Scale, the Self-Blame Scale, the Help-Seeking Scale, the Problem-focused / Help-Seeking Scale and the Wishful thinking / Escape Scale all increase from time 1 to time 2 and decrease from time 2 to time 3. The opposite pattern is observed for the Emotional Withholding Scale, that is a decrease from time 1 to time 2 and an increase from time 2 to time 3. The Duke-UNC Health Profile indicates that the Physical Function and the Emotional Function Scales decrease from time 1 to time 2 and increase from time 2 to time 3. The Symptom Status Scale and Social Function 
Scale increase across time, implying an improvement in health status on these dimensions across time. Only immune parameters at Time 3 can be commented on. These results indicate an overall suppressed immune system as indicated by the low CD 4 cell levels and raised White Cell Count, Neutrophil and Monocyte \% and Absolute values. The fact that this participant was HIV positive prior to the rape might account for the suppressed immune system.

\section{Participant 5}

Observation of the psychological results indicate variable patterns across time. An increase is noticed from time 1 to time 2 on the Somatization Scale, the Paranoid Ideation Scale and the Hostility Scale. This is followed by a decrease from time 2 to time 3 on these scales. The opposite pattern is observed on the Depression Scale, that is there is a decrease form time 1 to time 2 and an increase from time 2 to time 3. The Obsessive-Compulsive Scale, the Interpersonal Scale and the Phobic Anxiety Scale all increase across time 1,2 and 3. An increase is also noted on the Anxiety Scale and the Psychotism Scale from time 1 to time 2. These scales remains constant form time 2 to time 3. The Intrusion Scale and the Avoidance scale, both sub-scales of the Revised Impact of Events Scale increase from time 1 to time 2 and decrease from time 2 to time 3. The Ways of Coping employed by this participant appear consistent across time. The DukeUNC Health Profile reveals a lowered overall health status, even though the Physical Function Scale is raised at time 2 and time 3 and the Symptom Status Scale is raised at time 2. This participant has a poor health status and does not appear to be coping with social responsibilities. Observation of the immune parameters indicate a overall low $\mathrm{CD} 4$ cell count level. This is due to the participant being HIV positive. Therefore is participant is expected to have a suppressed immune system.

\subsubsection{Analysis of blood measures across time}

In evaluating the relationship between rape and the immune system it is important to consider the changes in the immune parameters across time. This is measured at time 1 ( $\mathrm{T} 1$ - five days after the rape ), time 2 ( T2 - fifteen days after the rape), time 3 ( T3 - thirty five days after the rape). It is not possible to obtain a baseline measure of immune parameters prior to the rape. The measurement obtained by flow cytometery, used in this research, provides quantitative information only. Whether or not a cell population is capable of carrying out a function cannot 
be ascertained. Rabin ( 1999) states that any further explanation beyond this presentation of data will lead to speculation that cannot be supported. The reader is referred to appendix 1 for an explanation of the functions of different cells measured. A brief discussion of cell-mediated immunity is also included. Table 3 indicates the changes in the cell numbers at time 1, time 2 and time 3. Figure 3 indicates the changes in immune parameters across time. 
TABLE 3: Table showing mean blood measures at Time 1,2,3 and normative levels of the various blood measures.

\begin{tabular}{|l|l|l|l|l|l|l|l|}
\hline $\begin{array}{l}\text { Blood Cell } \\
\text { Count }\end{array}$ & Time 1 & SD & Time 2 & SD & Time 3 & SD & $\begin{array}{l}\text { Normative } \\
\text { Measures }\end{array}$ \\
\hline CD 3 \% & 76.625 & 10.59 & 75.85 & 11.33 & 77.84 & 10.37 & - \\
\hline CD 3 absolute & 1746 & 851.1 & 1555.3 & 588.7 & 1576.4 & 406.6 & $800-2800$ \\
\hline CD 4 \% & 39.125 & 16.9 & 35.625 & 12.21 & 38.64 & 13.45 & - \\
\hline CD 4 absolute & 1006.25 & 731.8 & 941.25 & 682.9 & 816.4 & 388.5 & $550-1955$ \\
\hline CD 8 \% & 33.45 & 16.21 & 37.525 & 18.92 & 38.82 & 19.29 & - \\
\hline CD 8 absolute & 699.75 & 309.7 & 832.25 & 459.6 & 848.4 & 392.9 & $250-1200$ \\
\hline platelets & 321 & 102.5 & 319 & 102.4 & 282.4 & 82.1 & $150-450^{*} 10^{\wedge} 9 / 1$ \\
\hline white cell count & 6.375 & 0.359 & 5.525 & 1.335 & 6.8 & 3.142 & $4.0-11.0^{*} 10^{\wedge} 9 / 1$ \\
\hline neutrophils & 52.35 & 22.37 & 44.125 & 17.55 & 49.88 & 16.74 & $40-75 \%$ \\
\hline neutrophils abs. & 3.375 & 1.55 & 2.35 & 1.316 & 3.64 & 2.638 & $2.0-7.5 * 10^{\wedge} 9 / 1$ \\
\hline lymphocytes & 36.125 & 18.02 & 41.425 & 17.55 & 35.98 & 13.22 & $20-45 \%$ \\
\hline lymphocytes ab & 2.275 & 1.053 & 2.425 & 1.408 & 2.28 & 1.026 & $1.5-4.0 * 10^{\wedge} 9 / 1$ \\
\hline monocytes & 8.55 & 1.455 & 9.125 & 1.524 & 10.48 & 2.820 & $2-10 \%$ \\
\hline monocytes abs. & 0.55 & 0.129 & 0.5 & 0.148 & 0.7 & 0.324 & $0.5-1.5^{*} 10^{\wedge} 9 / 1$ \\
\hline eosinophils \% & 1.8 & 1.470 & 4.7 & 3.297 & 2.6 & 1.920 & $0.0-6 \%$ \\
\hline & & & & & & & \\
\hline
\end{tabular}

Figure 3 : Measures of the Immune Parameters Across Time 1, 2 and 3

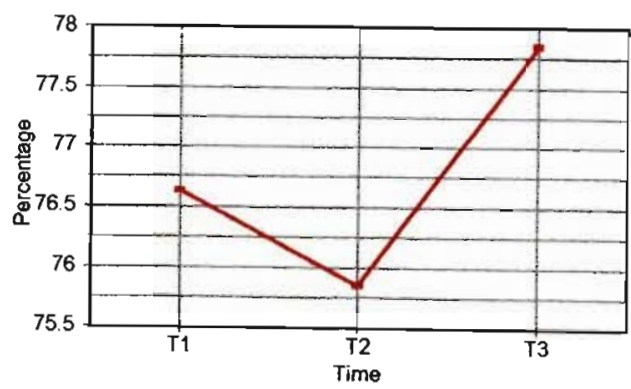

Figure 3a: The mean value of $\mathrm{CD} 3 \%$ across time

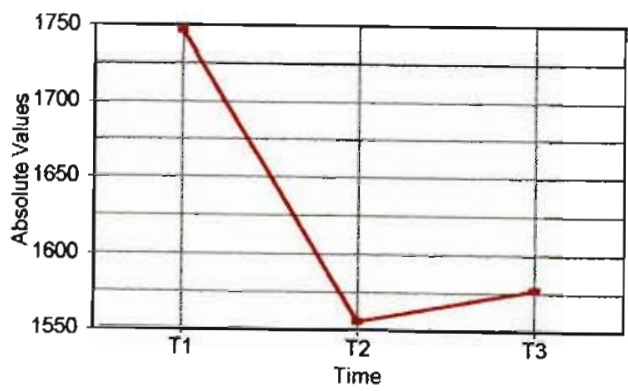

Figure 3b: The mean value of $\mathrm{CD} 3$ absolute across time 


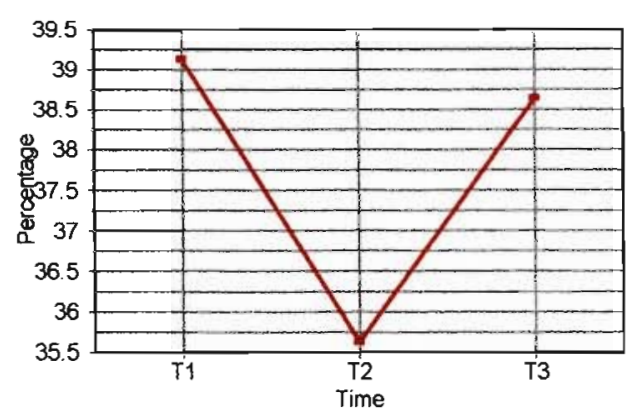

Figure $3 \mathrm{c}$ : The mean value of $\mathrm{CD} 4 \%$ across time

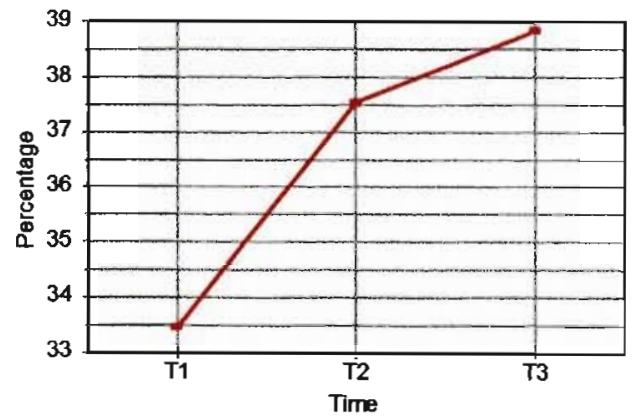

Figure 3e: The mean value of $\mathrm{CD} 8 \%$ across time

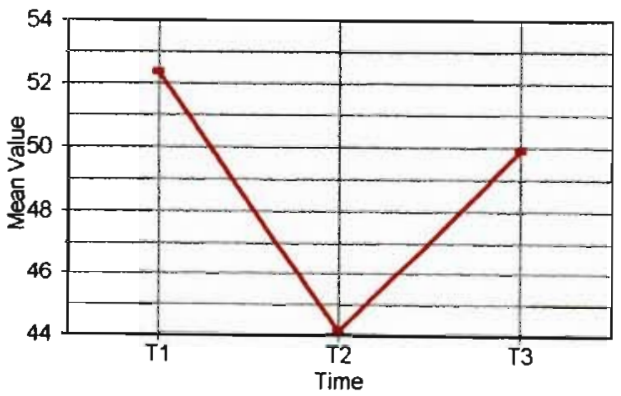

Figure 3g: The mean value of Neutrophils $\%$ across time

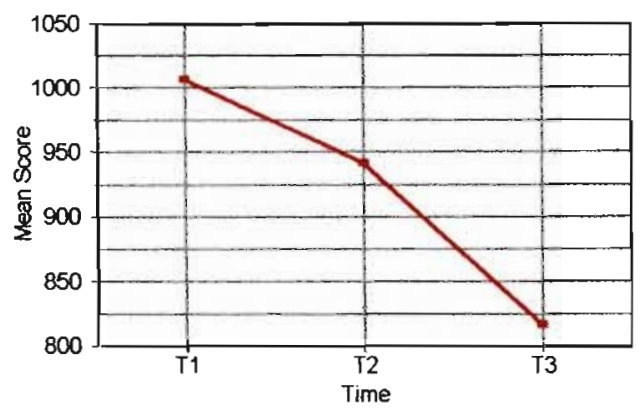

Figure 3d: The mean value of $\mathrm{CD} 4$ absolute across time

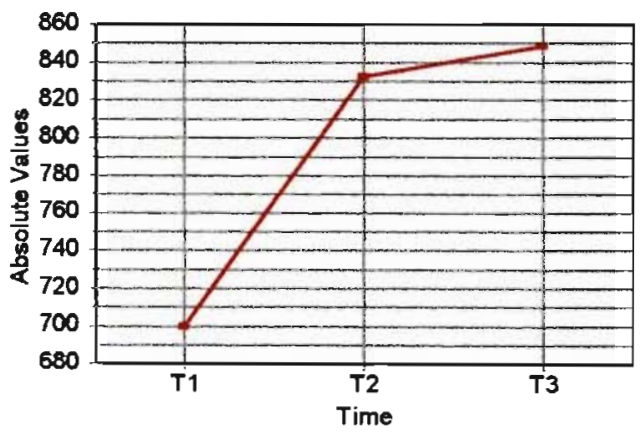

Figure 3f: The mean value of $\mathrm{CD} 8$ absolute across time

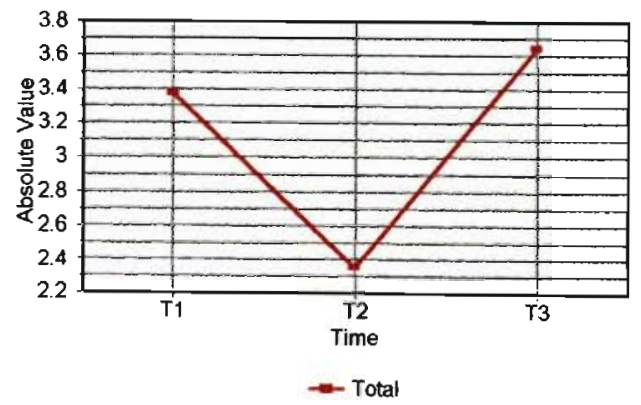

Figure 3h: The mean value of Neutrophils absolute across time 


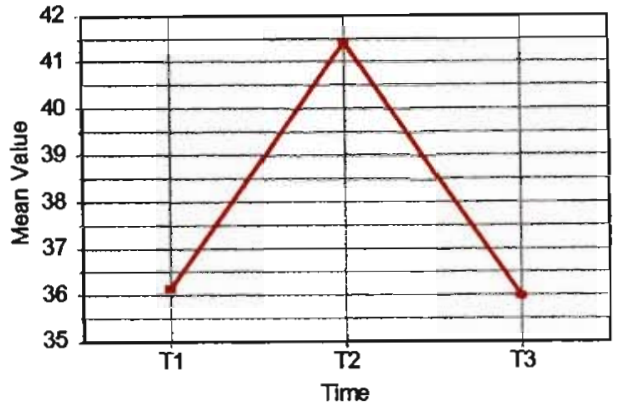

Figure 3i: The mean value of Lymphocyte \% across time

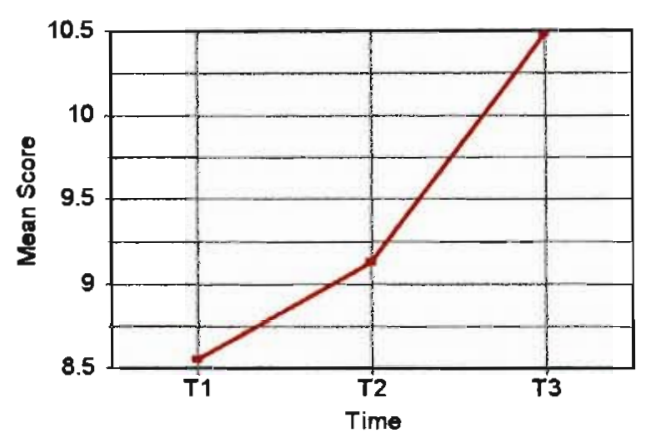

Figure 3k: The mean value of Monocytes \% across time

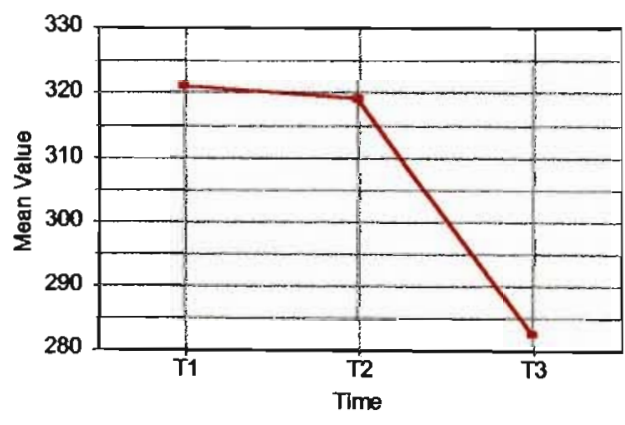

Figure 3m: The mean value of Platelets across time

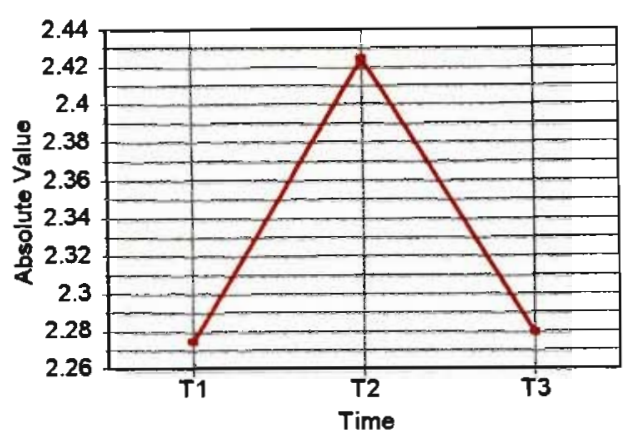

Figure 3j: The mean value of Lymphocyte absolute across time

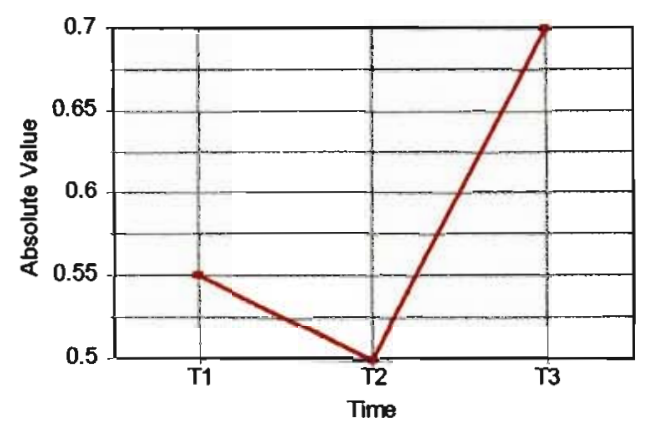

Figure 31: The mean value of Monocyte absolute across time

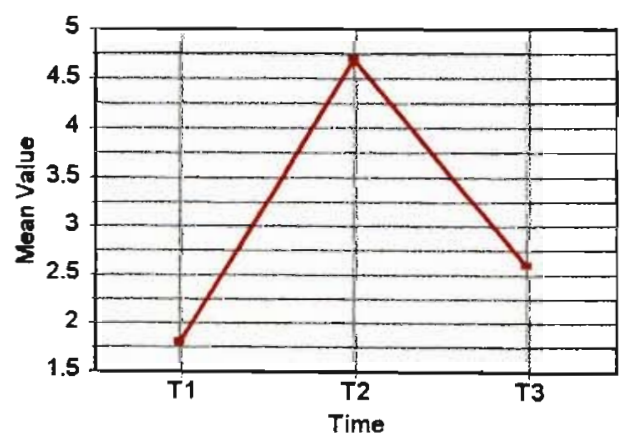

Figure $3 \mathrm{n}$ : The mean value of Platelets across time 


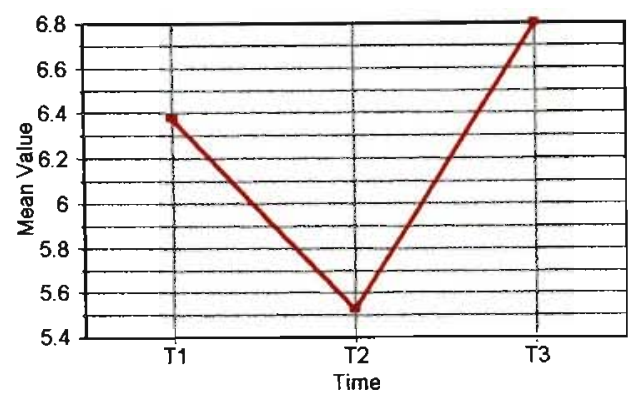

Figure 30: The mean value of White Cell Count across time

It is evident from table 2 that all the immune parameters are within the normative range of values, with the exception of monocyte percentage, at time 3 , that is 35 days after the rape. This abnormality of $0.48 \%$ suggests a presence of antigen or some foreign body. Friedman tests were carried out on the different blood measurements across the different times. Significant differences were obtained for the percentage of CD 4 cell numbers $(p=0.039)$ between time 1 and time 2 as well as between time 2 and time 3 . A significant difference $(p=0.039)$ was also noted for platelets between time 2 and time 3 (platelets are a haematological measure which aids the immune system, see appendix 1 ). Figure 3 indicates that the measures of $\mathrm{CD} 3 \%, \mathrm{CD} 3$ absolute, CD $4 \%$, neutrophils \%, neutrophils absolute, monocytes absolute and white cell count decrease between time 1 and time 2 and increase between time 2 and time 3 . The measures of lymphocytes $\%$, lymphocytes absolute and eosinophils increase between time 1 and time 2 and decrease between time 2 an time 3. CD 4 absolute and platelets increase across time, whereas CD $8 \%, C D$ 8 absolute and monocyte \% decrease between time 2 and time 3.

\subsubsection{Analysis of levels of distress across time}

The following data were obtained for levels of distress across times 1,2 and 3 . The mean scores and standard deviations are presented in Table 19, Appendix 8. 
Figure 4: Measures of Levels of Distress Across Time

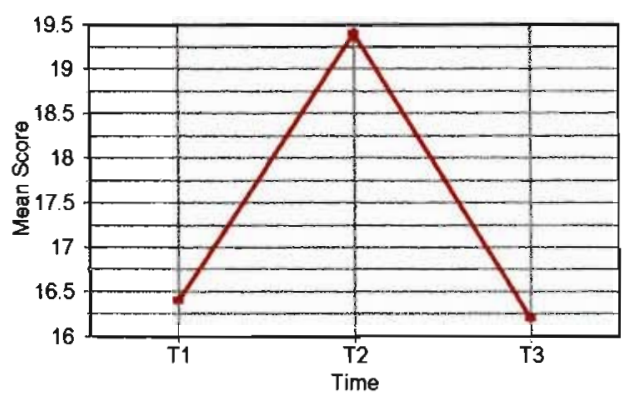

Figure 4a: Measure of the Somatization Scale Across Time

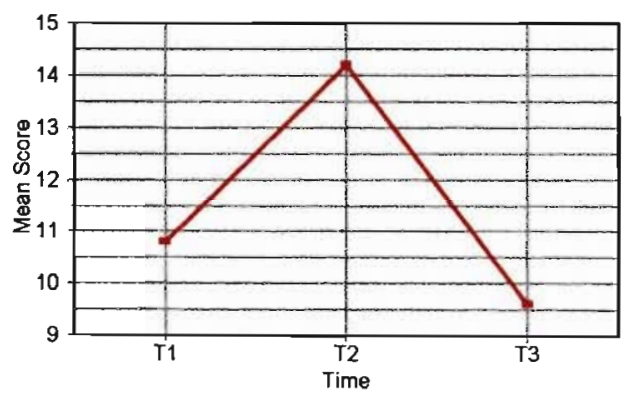

Figure 4c: Measure of the Interpersonal-Sensitivity Scale Across Time

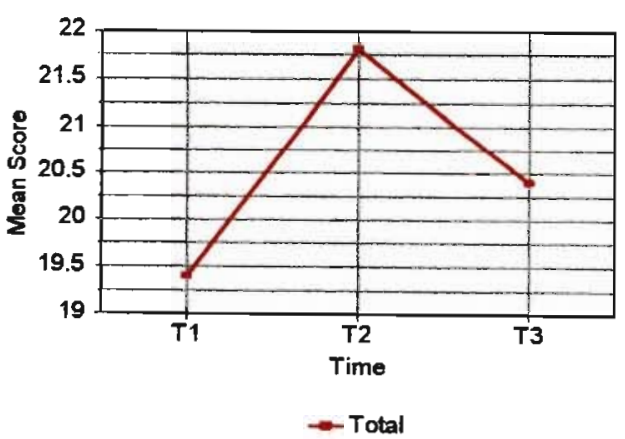

Figure 4e: Measure of the Anxiety Scale Across Time

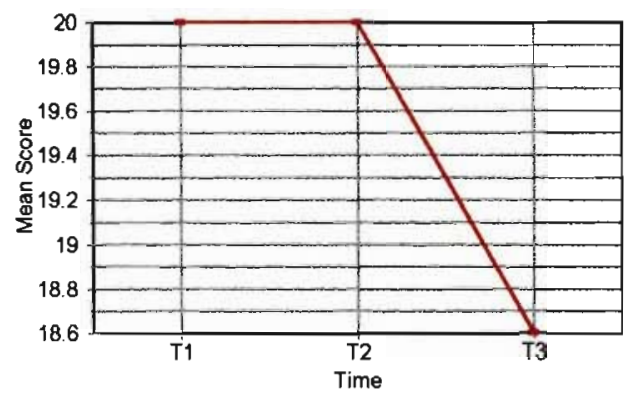

Figure 4 b: Measure of the Obsessive-Compulsive Scale Across Time

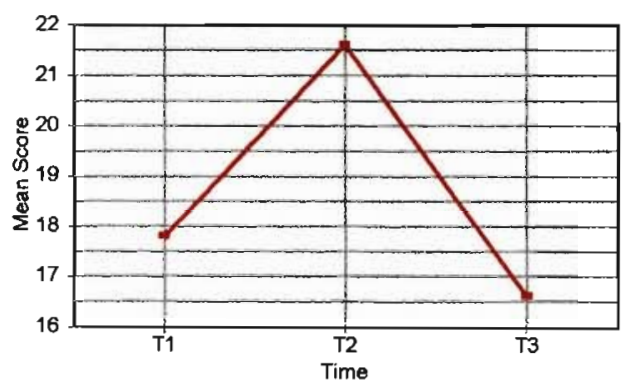

Figure 4d: Measure of the Depression Scale Across Time

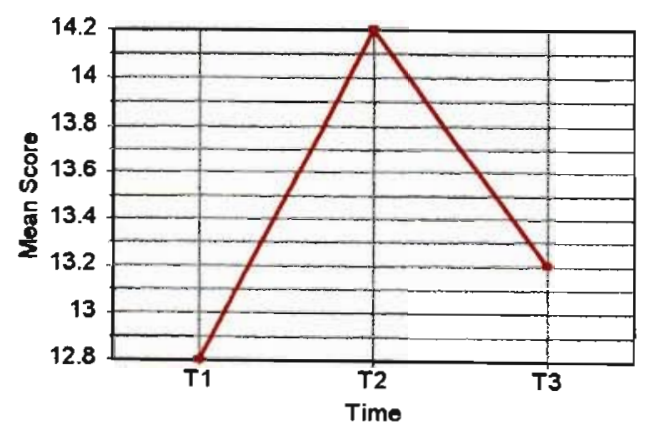

Figure 4f: Measure of the Hostility Scale Across Time 


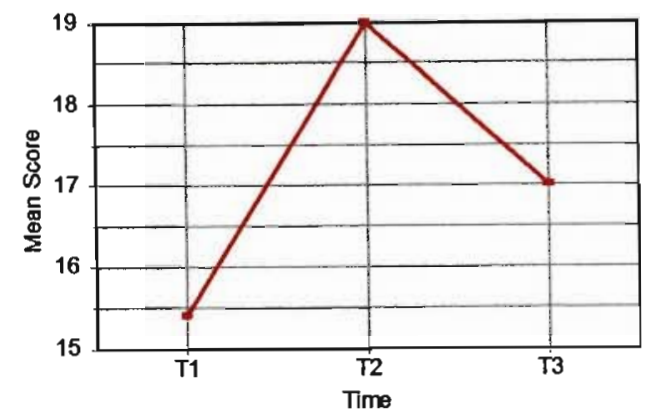

Figure 4g: Measure of the Phobic-Anxiety

Scale Across Time

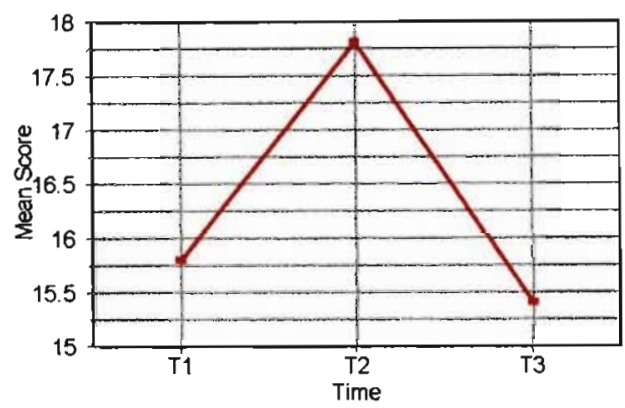

Figure 4i: Measure of the Paranoid-Ideation

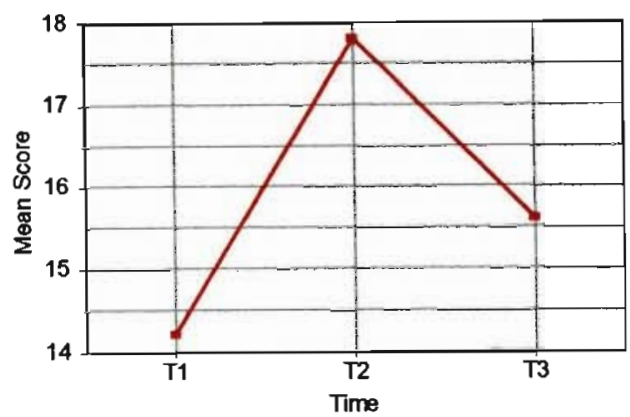

Figure 4h: Measure of the Psychotism Scale Across Time Scale Across Time

Friedman tests were carried out in order to assess the levels of distress at time 1, time 2 and time 3 . No significant differences in the levels of distress were noted across time. With the exception of the Obsessive-Compulsive Scale all the levels of distress illustrated in Figure 4 , reveal an increase in the levels of distress from time 1 to time 2. The Obsessive-Compulsive Scale remains the same at time 1 and time 2 . This is followed by a decrease in the measures of all levels of distress between time 2 and time 3 . 


\subsubsection{Analysis of impact of events across time}

The following data were obtained for the impact of the rape across times 1,2 and 3 .

Figure 5: Measure of the Impact of Events Scale Across Time

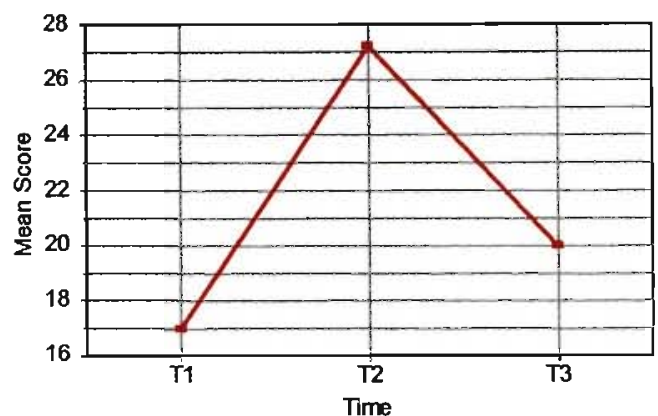

Figure 5a: Measure of the Avoidance Scale across time

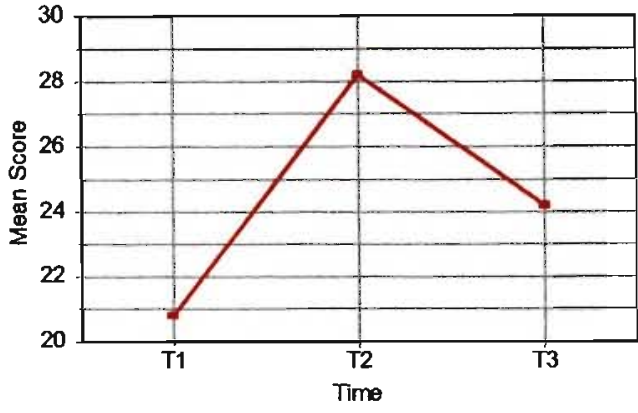

Figure 5b: Measure of the Intrusion Scale across time

Friedman tests were carried out in order to assess the levels of distress at time 1, time 2 and time 3 . No significant differences in the levels of distress were noted across time. The impact of the rape is measure on two scale the avoidance scale and the intrusion scale. Figure 5 indicates the increase between time 1 and time 2, and the decrease between time 2 and time 3 on the Avoidance Scale and the Intrusion Scale.

\subsubsection{Analysis of ways of coping across time}

The following data were obtained for the ways of coping across times 1,2 and 3 .

Figures 6: Measure of the Ways of Coping Across Time

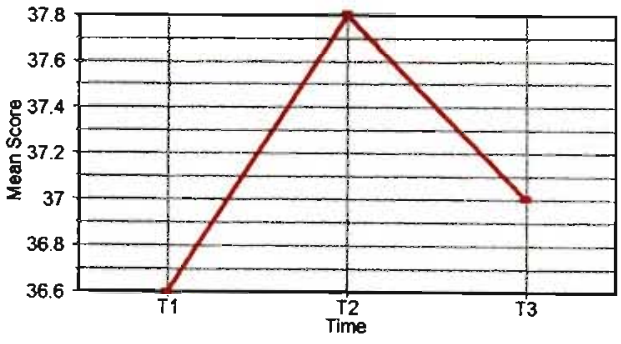

Figure 6a: Measure of the Wishful thinking/escape Scale Across Time

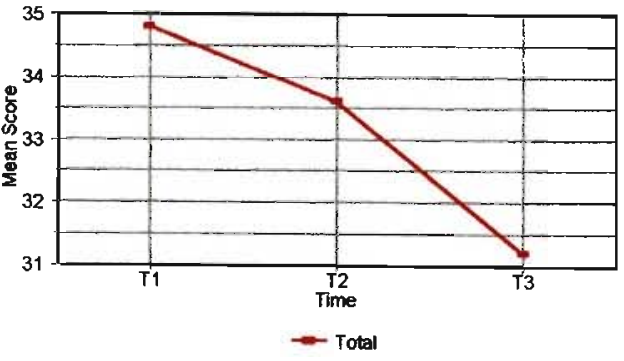

Figure 6b: Measure of the Problem-focused / Help-seeking Scale Across Time 


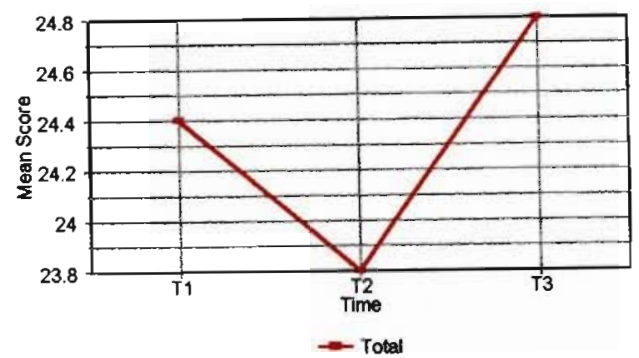

Figure 6c: Measure of the Emotional-withholding Scale Across Time

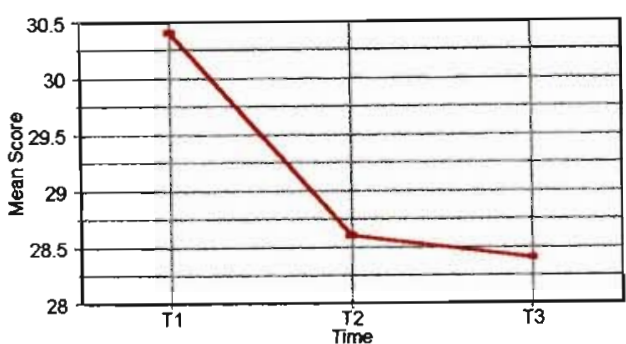

Figure 6e: Measure of the Acceptance Scale Across Time

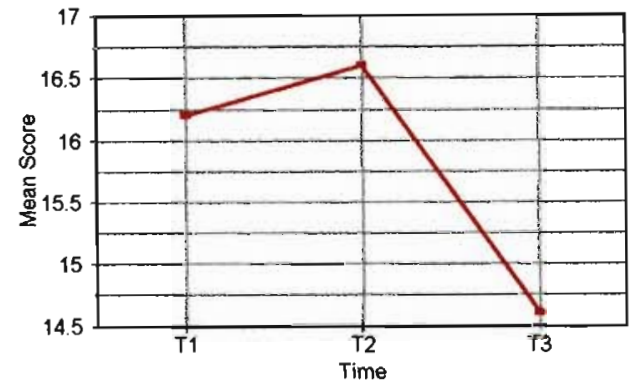

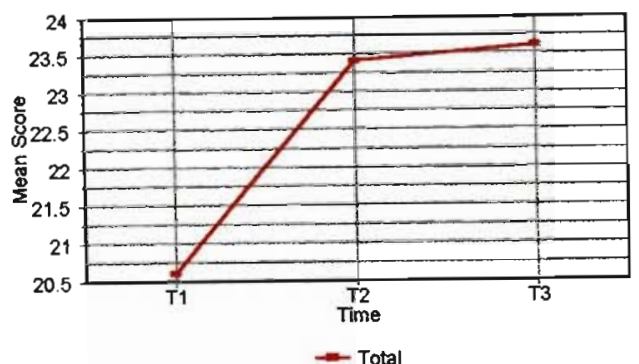

Figure 6d: Measure of the Self-Blame Scale Across Time

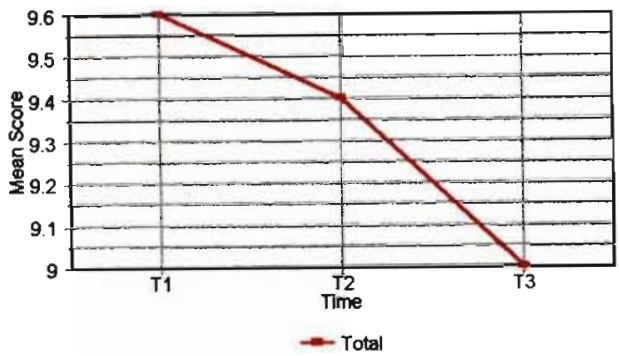

Figure 6f: Measure of the Help-Seeking Scale Across Time

Figure 6g: Measure of the Growth

Scale Across Time

Friedman tests were conducted on all the ways of coping scales. No significant differences in the ways of coping across time was obtained. However an observation of Figure 6 indicates changes in the mean values of coping styles used across time 1 , time 2 and time 3. 


\subsubsection{Analysis of Health Profile Across Time}

The following data indicate the changes in the measures of the health profile dimensions across

Figures 7: Measure of the Dimensions of the Health Profile Across Time

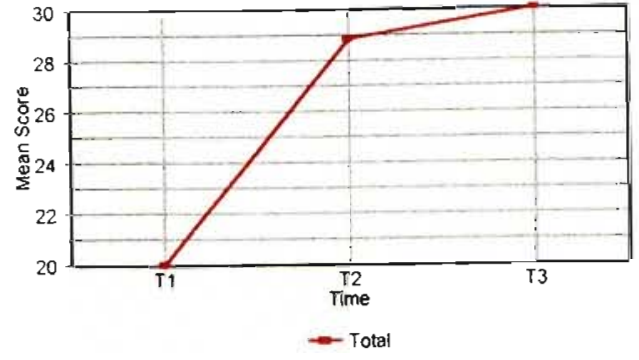

Figure 7a: Measure of the Symptom Status

Scale Across Time

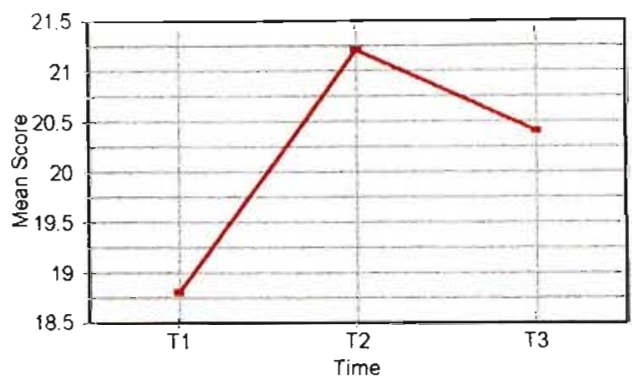

Figure 7c: Measure of the Physical Status Scale Across Time

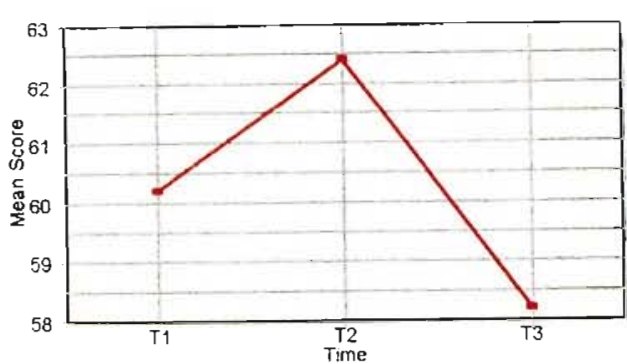

Figure 7b: Measure of the Emotional Status Scale Across Time

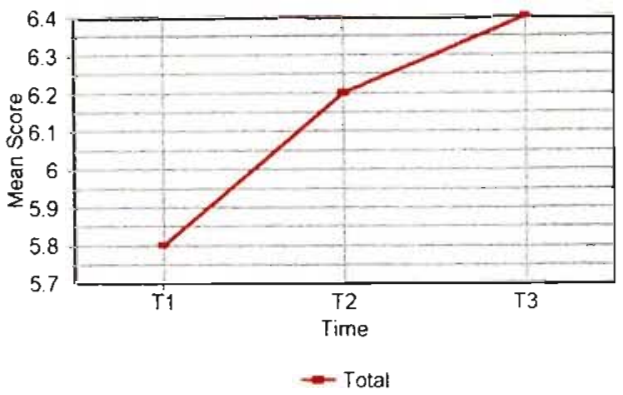

Figure 7d: Mcasure of the Social Function Scale Across Time

A person's health profile gives an indication of their overall well-being. Therefore it is necessary to analyse a rape survivors' health status which is measured along four dimensions which are symptom status, emotion status, physical status and social function across time. Friedman tests were carried out in order to assess the levels of distress at time 1 , time 2 and time 3 . No significant differences in the dimensions of the health profile were noted across time 


\subsection{The Relationship between Different Variables}

The following section looks at the relationship between the following variables. This includes the relationship between recent life change events and immune parameters; levels of distress and immune parameters; ways of coping and immune parameters; health profile and immune parameters; health profile and life change events, and finally levels of distress and ways of coping.

\subsubsection{Relationship between Life Change Events and Immune Parameters.}

The literature in PNI suggests that life change events have an effect on the immune system. Therefore it is important to assess whether or not life change events prior to rape have a relationship with the immune parameters being measured. Pearson Product Moment correlations were calculated between life change events, excluding rape, and immune parameters measured at time 1, time 2 and time 3 . No significant correlations were found between life change events and immune parameters measured at time 1 , time 2 and time 3 . This suggests that life change events do not have a relationship with the immune parameters measured in this study.

\subsubsection{Relationship between Levels of Distress and Immune Parameters}

Rape is a highly traumatic event which can cause a survivor to experience distress. It has been previously suggested in the literature review, that there are stress induced changes to the immune system. The stress induced changes of rape on the immune system, to date are not documented. It has been hypothesized that there is a relationship between Levels of Distress and Immune Parameters. The analysis of data aims to investigate the relationship between various levels of distress and immune parameters. The levels of distress include all the scales on the Brief Symptom Inventory and the scales of the Impact of Events Questionnaire. Pearson product moments were calculated to investigate the relationship between levels of distress and immune parameters. Only the significant correlations are presented in Table 4,5,6 and 7 . 
Table 4 : Relationship between level of distress and blood measurements $n=12$ [ $T$ 1 ]

\begin{tabular}{|l|l|l|l|}
\hline Levels of Distress & $\begin{array}{l}\text { Blood } \\
\text { measurement }\end{array}$ & $\mathbf{r}$ & $\mathbf{p}$ \\
\hline intrusion & CD 4 absolute & -0.603 & 0.049 \\
\hline intrusion & neutrophil \% & 0.617 & 0.043 \\
\hline intrusion & lymphocyte \% & -0.630 & 0.038 \\
\hline somatization & basophil \% & 0.904 & 0.002 \\
\hline sensitivity & neutrophil absolute & 0.697 & 0.017 \\
\hline anxiety & white cell count & 0.645 & 0.032 \\
\hline depression & neutrophil \% & 0.639 & 0.034 \\
\hline depression & neutrophil absolute & 0.606 & 0.048 \\
\hline depression & lymphocyte \% & -0.632 & 0.037 \\
\hline depression & basophil \% & 0.891 & 0.003 \\
\hline phobic anxiety & neutrophil absolute & 0.647 & 0.031 \\
\hline phobic anxiety & eosinophil \% & -0.705 & 0.015 \\
\hline phobic anxiety & basophil \% & 0.714 & 0.047 \\
\hline paranoid ideation & neutrophil absolute & 0.673 & 0.023 \\
\hline psychotism & neutrophil absolute & 0.713 & 0.014 \\
\hline & & & \\
\hline & & 0.037 \\
\hline
\end{tabular}


Table 5 : Relationship between level of distress and blood measurements $n=5$ [ T 1 ]

\begin{tabular}{|l|l|l|l|}
\hline Levels of Distress & $\begin{array}{l}\text { Blood } \\
\text { measurement }\end{array}$ & $\mathbf{p}$ & $\mathbf{p}$ \\
\hline sensitivity [T1] & CD 8 absolute & -0.947 & 0.053 \\
\hline intrusion [T1] & eosinophils \% & -0.990 & 0.010 \\
\hline somatisation [T1] & monocytes absolute & -0.948 & 0.052 \\
\hline obsessive [T1] & monocytes absolute & -0.947 & 0.053 \\
\hline depression [T1] & eosinophils \% & -0.973 & 0.027 \\
\hline depression [T1] & eosinophils absolute & -0.957 & 0.043 \\
\hline sensitivity [T1] & eosinophils \% & -0.961 & 0.039 \\
\hline Paranoid [T1] & eosinophils \% & -0.949 & 0.051 \\
\hline hostility [T1] & eosinophils \% & -0.994 & 0.006 \\
\hline hostility [T1] & eosinophils absolute & -0.992 & 0.008 \\
\hline psychotism [T1] & eosinophils \% & -0.973 & 0.027 \\
\hline
\end{tabular}

Table 6: Relationship between level of distress and blood measurements $n=5[$ T 2 ]

\begin{tabular}{|l|l|l|l|}
\hline Levels of Distress & $\begin{array}{l}\text { Blood } \\
\text { measurement }\end{array}$ & $\mathbf{r}$ & $\mathbf{p}$ \\
\hline sensitivity [ T1] & CD 8 absolute & -0.970 & 0.030 \\
\hline hostility [T1] & CD 3 absolute & -0.955 & 0.045 \\
\hline psychotism [T1] & CD 8 absolute & -0.962 & 0.038 \\
\hline intrusion [ T 1] & white cell count & -0.960 & 0.040 \\
\hline depression [ T1] & white cell count & -0.956 & 0.044 \\
\hline intrusion [ T 2] & lymphocyte absolute & -0.951 & 0.049 \\
\hline hostility [T1] & lymphocyte absolute & -0.976 & 0.024 \\
\hline anxiety [ T2] & platelets & 0.970 & 0.030 \\
\hline
\end{tabular}


Table 7: Relationship between level of distress and blood measurements $n=5$ [ T 3 ]

\begin{tabular}{|c|c|c|c|}
\hline Levels of Distress & $\begin{array}{l}\text { Blood } \\
\text { measurement }\end{array}$ & $\mathbf{r}$ & $\mathbf{p}$ \\
\hline intrusion [T1] & CD 8 absolute & -0.928 & 0.023 \\
\hline somatization [T1] & CD 8 absolute & -0.937 & 0.019 \\
\hline sensitivity $[\mathrm{T} 1]$ & CD 8 absolute & -0.883 & 0.047 \\
\hline hostility [T1] & CD 8 absolute & -0.885 & 0.046 \\
\hline psychotism [T1] & CD 8 absolute & -0.887 & 0.045 \\
\hline avoidance [T1] & lymphocytes \% & -1.000 & 0.016 \\
\hline intrusion [T1] & monocytes absolute & -0.970 & 0.006 \\
\hline hostility [T1] & monocytes absolute & -0.994 & 0.001 \\
\hline intrusion [T2] & $\mathrm{CD} 3 \%$ & 0.980 & 0.004 \\
\hline phobic anxiety [T2] & $\mathrm{CD} 4 \%$ & 0.883 & 0.047 \\
\hline phobic anxiety [T2] & $\mathrm{CD} 8 \%$ & -0.906 & 0.034 \\
\hline anxiety [T2] & platelets & 0.962 & 0.009 \\
\hline avoidance [T3] & $\mathrm{CD} 4 \%$ & 0.901 & 0.037 \\
\hline avoidance $[\mathrm{T} 3]$ & CD 4 absolute & 0.964 & 0.008 \\
\hline somatization [T3] & CD $3 \%$ & -0.922 & 0.026 \\
\hline anxiety [T3] & CD 8 absolute & -0.942 & 0.017 \\
\hline hostility [T3] & $\mathrm{CD} 3 \%-0.890$ & -0.890 & 0.043 \\
\hline somatization [T3] & platelets & 0.970 & 0.006 \\
\hline obsessive [T3] & monocytes \% & 0.913 & 0.030 \\
\hline obsessive [T3] & eosinophils \% & -0.998 & 0.002 \\
\hline sensitivity [T3] & eosinophils \% & -0.997 & 0.003 \\
\hline hostility [T3] & eosinophils absolute & -0.994 & 0.006 \\
\hline phobic anxiety [T3] & lymphocytes abs. & -0.970 & 0.030 \\
\hline psychotism [T3] & monocytes \% & 0.944 & 0.016 \\
\hline psychotism [T3] & eosinophils \% & -0.998 & 0.002 \\
\hline
\end{tabular}


The following is an interpretation of the relationships presented in the Tables 4,5,6 and 7.

\section{6,3.2.1 Relationship between levels of distress and CD 4}

It is evident from Table 4 (time 1 measure) that a significant negative correlation exists between CD 4 absolute and the Intrusion Scale. There is no significant relationship at time 2 and time 3. A significant positive relationship is noted between CD 4 absolute and Avoidance Scale at time 3 (Table 7). Therefore a measure of subjective distress, experienced by rape survivors demonstrates a significant association with CD 4 absolute numbers. The Avoidance Scale has a positive significant relationship with $\mathrm{CD} 4 \%$ at time 1 (see Table 4). A significant positive correlation is also noted between the Phobic Anxiety Scale measured at time 2 and CD $4 \%$ measured at time 3 , as indicated in Table 7.

\subsubsection{Relationship between levels of distress and neutrophils}

Table 4 reveals a significant positive correlation between the Intrusion Scale and neutrophil \%, measured at time 1. There is a significant positive correlation between neutrophil $\%$ and the Depression Scale measured at time 1, (see Table 4). It is evident from table 3 that a significant positive correlation exists between the Interpersonal Sensitivity Scale and neutrophil absolute. The Depression Scale and neutrophil absolute have a significant positive correlation at time 1, see Table 4. A significant positive correlation exists between the Phobic Anxiety Scale and neutrophil absolute at time 1, (see Table 4). Table 4 also indicates that the Paranoid Ideation Scale and neutrophil absolute have a significant positive correlation with each other, at time 1 . A significant positive correlation is evident between the Psychotism Scale and the neutrophil absolute measure, at time 1 (see Table 4).

\subsubsection{Relationship between levels of distress and lymphocytes}

Table 4 indicates significant negative relationships between lymphocytes $\%$ and the Intrusion Scale, as well as a significant negative relationship between lymphocyte $\%$ and the Depression Scale. This is not significant when the sample size is decreased to 5 at time 1,2 and 3 . There is a significant negative correlation between the Avoidance Scale measured at time 1 and lymphocyte \%, measured at time 3 (see Table 7). At time 2, the Intrusion Scale has a significant negative relationship with lymphocyte absolute (see Table 6). Significant negative correlations are 
evident between the Hostility scale and lymphocyte absolute, as well as the Phobic Anxiety scale and lymphocyte absolute. Table 6 also indicates a significant negative correlation between the Hostility Scale, measured at time 1 and lymphocyte absolute measured at time 2.

\subsubsection{Relationship between levels of distress and $\mathrm{CD} 8$}

There are significant negative correlations between the Interpersonal Sensitivity Scale measured at time 1 and CD 8 absolute measured at time 1, time 2 and time 3 (see Tables 5,6 and 7).

A significant negative correlation exists between CD 8 absolute and the Anxiety Scale at time 3 (see Table 7). The Somatization Scale, the Interpersonal Sensitivity Scale, the Hostility Scale and the Psychotism Scale all measured at time 1 demonstrates a significant negative relationship with CD 8 absolute, measured at time 2 (see Table 7). There is a significant negative correlation between the Phobic Anxiety Scale measured at time 2 and $\mathrm{CD} 8 \%$ measured at time 3, as indicated in Table 7.

\subsubsection{Relationship between levels of distress and CD 3}

At time 3, 35 days after the rape, a significant negative correlation exists between $\mathrm{CD} 3 \%$ and the Somatization Scale (see Table 7). A significant positive correlation exists between the Intrusion Scale measured at time 2 and $\mathrm{CD} 3 \%$ measured at time 3 (see Table 7).

\subsubsection{Relationship between levels of distress and eosinophils}

According to Table 5, at time 1, the Depression Scale has a significant negative correlation with osinophils absolute. A significant negative correlation is also found between the Hostility Scale and eosinophil absolute (see Table 5). At time 1 the Phobic Anxiety Scale has a significant negative relationship with eosinophil \% (see Table 4). This exists when the sample size is 12. However when the sample size is reduced to 5 , at time 1, the Intrusion Scale, the Depression Scale, the Interpersonal Sensitivity Scale, the Paranoid Ideation Scale, the Hostility Scale and the Psychotism Scale all have significant negative correlations with eosinophil \% as indicated in Table 5. At time 3, the Obsessive Compulsive Scale has a significant negative correlation with eosinophil \% (see Table 7). Table 7 also indicates a significant negative correlation with the Interpersonal Sensitivity Scale and eosinophil \%, at time 3. The Psychotism Scale also has a significant negative relationship with eosinophil \%, as indicated in Table 7. 


\subsubsection{Relationship between levels of distress and monocytes}

A significant negative correlation exists between the Obsessive-Compulsive Scale and monocyte absolute at time 1 (see Table 5). The Somatization Scale and monocyte absolute also have a significant negative correlation at time $l$ as indicated in table 5 . No other significant relationships were established at time 2 and time 3. A significant negative correlation is noted between the Intrusion Scale measured at time 1 and monocyte absolute measured at time 3 (see Table 7). Similarly the Hostility Scale measured at time 1 and monocyte absolute measured at time 3 have a significant negative relationship (see Table 7). At time 3 monocyte \% has a significant positive relationship with the Obsessive-Compulsive Scale (see Table 7). It is also evident from Table 7 that a significant positive correlation exists between the Psychotism Scale and monocyte \% at time 3.

\subsubsection{Relationship between levels of distress and white cell count}

At time 1 the Anxiety Scale has a significant positive correlation with white cell count (see Table 4). There is a significant negative correlation between the intrusion scale measured at time 1 and white cell count measured at time 2 (see Table 6). A significant negative correlation also exists between the depression scale measured at time 1 and white cell count measured at time 2 (see table 6).

\subsubsection{Relationship between levels of distress and platelets}

At time 1, no significant correlations are noted between levels of distress and platelets. However at time 2 the Anxiety has a significant and positive correlation with platelets (see Table 6) and at time 3 the Somatization Scale has a significant and positive correlation with platelets (see Table 7). There is a significant and positive correlation between the Anxiety Scale measured at time 2 and platelet measurements at time 3 , as indicated in Table 7 .

It is apparent from the results that specific levels of distress measured by the various scales of the Brief Symptom Inventory and the Impact of Events Scale have significant relationships with specific immune parameters. The direction of the relationship depends on the levels of distress and the immune parameters. Therefore, as hypothesized, there is a significant relationship between specific immune parameters and specific levels of distress. 


\subsubsection{Relationship between Coping and Immune Parameters}

The traumatic experience of rape elicits various coping strategies, which can be broadly classified as emotion-focused and problem-focused ways of coping. This study also focuses on the coping strategies employed by rape survivors across a 35 day period. The literature in psychoneuroimmunology and coping to date has not included the relationship between a rape survivors ways of coping and immune parameters. In this study the relationship is explored by Pearson Product Moment Correlations. Only the significant correlation are included in Tables $8,9,10$ and 11 .

The high correlations raised some concern, therefore scatter plots were carried out to establish the reason for the high positive and negative correlations. The scatterplots are available from the researcher for perusal. Some of the high correlations were due to the effect of extreme values reported by one participant. Repeating this research with a larger sample size would certainly provide more evidence about the relationship between ways of coping scales and different immune parameters. The small sample size also decreases the variability of the results, therefore resulting in the high correlations. The data analysis was carried out initially on a sample size of 12 at time 1. This sample size was then reduced to 5 , so that the data analysis could be carried out on the same sample size across time. Pearson Product Moment correlations were calculated on this sample size to assess the relationship between these variables across time 1,2 and 3 . The significant correlations are presented in Table 8,9,10 and 11. 
Table 8 : The relationship between Coping Scales and Blood Measurements [ $n=12$ ] Time 1

\begin{tabular}{|l|l|l|l|}
\hline Coping Scale & $\begin{array}{l}\text { Blood } \\
\text { Measurement }\end{array}$ & $\mathbf{p}$ & $\mathbf{p}$ \\
\hline emotion withholding & CD 4 \% & -0.642 & 0.033 \\
\hline emotion withholding & CD 4 absolute & -0.631 & 0.037 \\
\hline emotion withholding & neutrophils & 0.643 & 0.033 \\
\hline emotion withholding & neutrophils absolute & 0.608 & 0.047 \\
\hline emotion withholding & lymphocytes & -0.671 & 0.024 \\
\hline problem & neutrophils & 0.663 & 0.026 \\
\hline problem & lymphocytes & -0.649 & 0.031 \\
\hline problem & lymphocytesabsolute & -0.617 & 0.043 \\
\hline self blame & CD 4 \% & -0.739 & 0.009 \\
\hline self blame & CD 4 absolute & -0.728 & 0.011 \\
\hline self blame & neutrophils & 0.731 & 0.011 \\
\hline self blame & neutrophils absolute & 0.748 & 0.008 \\
\hline self blame & lymphocytes & -0.786 & 0.004 \\
\hline help seeking & CD 3 absolute & -0.664 & 0.028 \\
\hline help seeking & CD 4 absolute & -0.753 & 0.007 \\
\hline help seeking & neutrophils & 0.721 & 0.005 \\
\hline help seeking & lymphocytes & -0.731 & -0.774 \\
\hline help seeking & lymphocytesabsolute & -012 \\
\hline
\end{tabular}


Table 9 : The relationship between Coping Scales and Blood Measurements $[n=5]$ Time 1

\begin{tabular}{|l|l|l|l|}
\hline Coping Scale & $\begin{array}{l}\text { Blood } \\
\text { Measurement }\end{array}$ & $\mathbf{r}$ & $\mathbf{p}$ \\
\hline acceptance & CD 8 \% & 0.999 & 0.025 \\
\hline emotion & CD 4 & -0.998 & 0.040 \\
\hline emotion & neutrophil absolute & 0.999 & 0.024 \\
\hline emotion & neutrophil absolute & 0.997 & 0.047 \\
\hline help seeking & CD 4 Absolute & -0.980 & 0.020 \\
\hline help seeking & neutrophils absolute & 0.972 & 0.028 \\
\hline help seeking & lymphocytes \% & -0.978 & 0.022 \\
\hline help seeking & lymphocytes & -0.967 & 0.033 \\
\hline & & & \\
\hline
\end{tabular}

Table 10 : Relationship between Coping Scales and Blood Measurements [ $\mathrm{n}=5]$ Time 2

\begin{tabular}{|l|l|l|l|}
\hline Coping Scale & $\begin{array}{l}\text { Blood } \\
\text { Measurement }\end{array}$ & $\mathbf{r}$ & $\mathbf{p}$ \\
\hline emotion [T 1] & lymphocyte absolute & -0.999 & 0.033 \\
\hline emotion [T 1] & CD 4 \% & -1.000 & 0.002 \\
\hline acceptance [T2] & CD 3\% & 0.989 & 0.011 \\
\hline growth [ T 2 ] & monocytes absolute & 0.980 & 0.020 \\
\hline emotion [T 2] & lymphocyte absolute & -1.000 & 0.016 \\
\hline acceptance [T3] & CD 3\% & 0.968 & 0.011 \\
\hline self-blame [ T 3 ] & CD 8 \% & 0.976 & 0.024 \\
\hline problem [ T 3] & eosinophils \% & 0.956 & 0.044 \\
\hline
\end{tabular}


Table 11 :Relationship between Coping Scales and Blood Measurements $[n=5]$ Time 3

\begin{tabular}{|c|c|c|c|}
\hline Coping scales & $\begin{array}{l}\text { Blood } \\
\text { Measurement }\end{array}$ & $\mathbf{r}$ & $\mathbf{p}$ \\
\hline emotion [ T1 ] & CD 4 absolute & -0.969 & 0.031 \\
\hline problem [ $\mathrm{T} 1]$ & CD 4 absolute & -0.929 & 0.022 \\
\hline help-seeking [ T1] & $\mathrm{CD} 4 \%$ & -0.946 & 0.015 \\
\hline help-seeking [ T1] & CD 4 absolute & -0.970 & 0.006 \\
\hline problem [ $\mathrm{T} 1]$ & lymphocyte absolute & -0.906 & 0.034 \\
\hline wishful thinking [T2] & $\mathrm{CD} 4 \%$ & -0.969 & 0.007 \\
\hline wishful thinking [T2] & CD 4 absolute & -0.910 & 0.032 \\
\hline wishful thinking [T2] & CD $8 \%$ & 0.922 & 0.026 \\
\hline acceptance [T2] & $\mathrm{CD} 3 \%$ & 0.972 & 0.006 \\
\hline self-blame [T2] & $\mathrm{CD} 4 \%$ & -0.889 & 0.044 \\
\hline acceptance [T2] & platelets & -0.883 & 0.047 \\
\hline growth [T2] & monocytes absolute & 0.914 & 0.030 \\
\hline avoidance [T3] & $\mathrm{CD} 4 \%$ & 0.901 & 0.037 \\
\hline avoidance [T3] & CD 4 absolute & 0.964 & 0.008 \\
\hline self-blame [T3] & $\mathrm{CD} 3 \%$ & 0.944 & 0.016 \\
\hline self-blame [T3] & CD $3 \%$ & 0.972 & 0.006 \\
\hline self-blame [T3] & platelets & -0.956 & 0.011 \\
\hline acceptance [T3] & monocytes \% & -0.957 & 0.011 \\
\hline acceptance [T3] & eosinophils \% & 0.992 & 0.008 \\
\hline problem [T3] & eosinophils $\%$ & 0.991 & 0.009 \\
\hline emotion & eosinophils absolute & 0.986 & 0.014 \\
\hline
\end{tabular}




\subsubsection{Relationship between ways of coping and CD 4}

Analysis of the results at time $1(n=12)$ indicates a significant negative correlation between the Self-Blame Scale and CD $4 \%$ (see Table 8) A significant negative correlation is also noted between Emotional Withholding and $\operatorname{CD} 4 \%$ at time 1 , as indicated in Table 8 . When the sample size is reduced to 5 at time 1, Emotional Withholding continues to have a significant negative correlation with CD $4 \%$ (see Table 9). There is a significant negative correlation between the Emotional Withholding scale measured at time 1 and CD $4 \%$ measured at time 2 (see Table 10). There is a significant negative correlation between the Help-Seeking Scale measured at time 1 and CD $4 \%$ measured at time 3 (see Table 11). A significant negative correlation also exists between the Wishful Thinking Scale measured at time 2 and CD $4 \%$ measured at time 3 (see Table 11). Table 11 also indicates a significant negative relationship between the Self-Blame Scale measured at time 2 and CD $4 \%$ measured at time 3 .

According to Table 8, the Self-Blame Scale has a significant negative correlation with CD 4 absolute at time 1. The Emotional Withholding scale also has a significant and negative correlation with $\mathrm{CD} 4$ absolute at time 1. A significant negative correlation exists between Help-Seeking Scale and CD 4 absolute at time 1 (see Table 8). Table 7 indicates significant negative correlations between the Problem-focused / Help-Seeking Scale (measured at time 1) and CD 4 absolute (measured at time 3), the Help-Seeking Scale (measured at time 1) and CD 4 absolute (measured at time 3) and the Emotional Withholding Scale (measured at time 1) and CD 4 absolute (measured at time 3). A significant negative correlation also exists between the Wishful Thinking Scale measured at time 2 and CD 4 absolute measured at time 3 (see Table 11).

\subsubsection{Relationship between ways of coping and lymphocytes}

A significant negative correlation exists between the Help-Seeking Scale and lymphocyte absolute at time 1 (Table 8 and 9 ). At time 1 there is also a significant negative correlation between the Problem-focused/ Help-Seeking Scale and lymphocyte absolute (see table 8). The Emotional Withholding Scale has a significant negative correlation with lymphocyte absolute at time 2 (see Table 10). There is also a significant negative correlation between the Emotional Withholding Scale measured at time 1 and lymphocyte absolute measured at time 2, as indicated in Table 10. A significant negative correlation also exists between the Problem-Solving Scale measured at time 
1 and lymphocyte absolute measured at time 3 (see Table 11).

There is a significant negative correlation between Help-Seeking and lymphocyte \% at time 1 (see Table 8 and 9). The Problem-focused/ Help-Seeking Scale also has a significant negative correlation with lymphocyte $\%$ at time 1 (see Table 9). At time 1 a significant negative correlation is noted between the Emotional Withholding Scale and lymphocyte \% as well as between the Selfblame scale and lymphocyte \%.

\subsubsection{Relationship between ways of coping and neutrophils}

The Emotional Withholding Scale, the Self-Blame Scale, the Problem-focused / Help-seeking Scale and the Help-seeking Scale all correlate significantly and positively with neutrophil \%, as indicated in Table 8 . When the number of participants is reduced to $n=5$ only the Emotional Withholding scale has a significant positive relationship with neutrophil \%. No significant correlations are noticed at time 2 and time 3 .

There is a significant positive correlation between the Self-blame Scale and neutrophil absolute, as indicated in Table 8 . Significant positive correlations also exist between the Emotional Withholding Scale and neutrophil absolute (see Table 8 and 9). Further positive correlations at time 1 are observed between the Help-seeking Scale and neutrophil absolute (see Table 9).

No significant correlations are noted at time 2 and time 3 between any Ways of Coping Scales and neutrophil absolute.

\subsubsection{Relationship between ways of coping and eosinophils}

At time 3, there exists a significant positive correlation between the Problem-Solving / HelpSeeking Scale and eosinophils (see Table 11). Table 11 also indicates a significant and positive correlation between the Acceptance Scale and eosinophils at time 3. There is a significant positive correlation between eosinophil \% measured at time 2 and the Problem-solving Scale measured at time 3 , as indicated in Table 10.

The Emotional Withholding Scale has a significant positive correlation with eosinophil absolute at time 3 as indicated in Table 11 . 


\subsubsection{Relationship between ways of coping and CD 8}

At time 1 a significant positive correlation exists between the Acceptance Scale and CD $8 \%$. This is only significant at time 1 (see Table 9). CD $8 \%$ measured at time 2 and the Self-blame Scale measured at time 3 correlate significantly and positively, as indicated in Table 10 . There is a significant positive correlation between the Wishful thinking Scale measured at time 2 and CD $8 \%$ measured at time 3 (see Table 11 ).

\subsubsection{Relationship between ways of coping and $\mathrm{CD} 3$}

A significant and positive correlation is noted between the Acceptance Scale and CD $3 \%$ at time 2 (see Table 10). The Self-blame Scale and CD $3 \%$ also have a significant positive correlation, at time 2 as indicated in Table 10. A significant positive correlation exists between CD $3 \%$ measured at time 2 and the Acceptance Scale measured at time 3 (see Table 10).

\subsubsection{Relationship between ways of coping and monocytes}

At time 2 the Growth Scale and monocyte absolute has a significant positive correlation, see Table 10. There is a significant positive correlation between the Growth Scale measured at time 2 and monocyte absolute measured at time 3 . At time 3 (see Table 11 ), a negative correlation is noted between monocyte \% and the Acceptance Scale.

\subsubsection{Conclusion}

It is therefore evident that both problem-focused and emotion-focused coping strategies have a significant relationship with various immune parameters. Therefore, as hypothesized, there is a significant relationship between ways of coping and immune parameters.

\subsubsection{Relationship between Health Profile and Recent Life Events}

Pearson product moment correlations were calculated to investigate the relationship between Health Profile and Recent Life Events across time. The aim of this analysis was to examine the possibility of whether the health profile reported was due to other life change events, besides rape. This analysis revealed no significant correlations. This therefore suggests that the health profile reported is not as a result of any other pre-existing life change events, except rape. 


\subsubsection{Relationship between Health Profile and Blood Measurements across time}

Pearson product moment correlations were calculated to assess whether there were any relationships between symptom status, physical function, emotional function and social function as assessed by the Duke-UNC Health Profile and immune parameters. The significant correlations between dimensions of the Health Profile and immune parameters are presented in Table 12,13, 14 and 15 below.

Table 12: Relationship between Duke Health Profile and Blood Measurements Time 1, $\mathrm{n}=12$

\begin{tabular}{|l|l|l|l|}
\hline $\begin{array}{l}\text { Health Profile } \\
\text { Scales }\end{array}$ & $\begin{array}{l}\text { Blood } \\
\text { measurements }\end{array}$ & r & p \\
\hline symptom status & white cell count & -0.733 & 0.010 \\
\hline social status & white cell count & -0.625 & 0.040 \\
\hline social status & neutrophil absolute & -0.719 & 0.013 \\
\hline
\end{tabular}

Table 13: Relationship between Duke Health Profile and Blood Measurements Time 1, $\mathrm{n}=5$

\begin{tabular}{|l|l|l|l|}
\hline $\begin{array}{l}\text { Health Profile } \\
\text { Scales }\end{array}$ & $\begin{array}{l}\text { Blood } \\
\text { measurements }\end{array}$ & r & p \\
\hline $\begin{array}{l}\text { emotional function } \\
{[\mathrm{T} 1]}\end{array}$ & CD $4 \%$ & 0.977 & 0.023 \\
\hline $\begin{array}{l}\text { emotional function } \\
{[\mathrm{T} 1]}\end{array}$ & white cell count & -0.993 & 0.007 \\
\hline
\end{tabular}


Table 14 : Relationship between Duke Health Profile and Blood Measurements Time $2, \mathrm{n}=5$

\begin{tabular}{|l|l|l|l|}
\hline $\begin{array}{l}\text { Health Profile } \\
\text { Scales }\end{array}$ & $\begin{array}{l}\text { Blood } \\
\text { measurements }\end{array}$ & $\mathbf{p}$ & $\mathbf{p}$ \\
\hline symptom status [T2] & CD 3\% & -0.948 & 0.052 \\
\hline $\begin{array}{l}\text { emotional function } \\
\text { [T2 ] }\end{array}$ & CD 4\% & -0.945 & 0.055 \\
\hline symptom status [T2] & CD 8\% & -0.995 & 0.005 \\
\hline social functioning & monocytes \% & -0.989 & 0.011 \\
\hline
\end{tabular}

Table 15: Relationship between Duke Health Profile and Blood Measurements Time $3, \mathrm{n}=5$

\begin{tabular}{|l|l|l|l|}
\hline $\begin{array}{l}\text { Health Profile } \\
\text { Scales }\end{array}$ & $\begin{array}{l}\text { Blood } \\
\text { measurements }\end{array}$ & $\mathbf{p}$ \\
\hline social functioning & CD 3 absolute & 0.900 & 0.037 \\
\hline symptom status [T2] & monocyte \% & 0.916 & 0.029 \\
\hline symptom status [T2] & eosinophils \% & -0.984 & 0.016 \\
\hline
\end{tabular}

\subsubsection{Relationship between the health profile and $\mathrm{CD} 4$}

At time 1 there is a significant positive correlation between the Emotional Function Scale and CD $4 \%$ (see Table 13). However at time 2, the Emotional Function Scale and CD $4 \%$ have a significant negative correlation (see Table 14).

\subsubsection{Relationship between the health profile and CD 3}

Table 14 reveals that a significant negative correlation exists between the Symptom Status Scale and $\mathrm{CD} 3 \%$ measured at time 2. The Social Functioning Scale and CD 3 absolute have a significant positive correlation at time 3 (see Table 15). 


\subsubsection{Relationship between the health profile and $\mathrm{CD} 8$}

There is a significant negative correlation between the Symptom Status Scale and CD $8 \%$ measured at time 2 (see Table 14).

\subsubsection{Relationship between the health profile and white cell count}

At time 1, the Symptom Status Scale and white cell count have a significant negative correlation as indicated in Table 12. A significant negative correlation also exists between the Social Function Scale and white cell count measured at time 1 (see Table 12). At time 2 the Emotional Function Scale and white cell count have a significant negative correlation (see Table 13).

\subsubsection{Relationship between the health profile and neutrophils}

There is a significant negative correlation between the Social Function Scale and neutrophil absolute, measured at time 1 (see Table 12).

\subsubsection{Relationship between the health profile and monocytes}

A significant negative correlation exists between the Social Function Scale and monocytes \% measured at time 2 (see Table 14). The Symptom Status Scale measured at time 2 has a significant positive relationship with monocyte \% measured at time 3 (see Table 15).

\subsubsection{Relationship between the health profile and eosinophil}

There is a significant negative correlation between the Symptom Status Scale measured at time 2 and the eosinophil \% measured at time 3 (see Table 15).

\subsubsection{Conclusion}

It is evident from the above significant correlations, that a significant relationship exists between a rape survivors heath profile and immune parameters. Therefore, the hypothesis which states that there is a relationship between a rape survivors health profile and immune parameters is confirmed. The strength of the relationship depends on dimension of the health profile and the immune parameter. 


\subsection{6, Relationship between Immune Parameters and Recent Life Changes}

Pearson Product Moment Correlations were carried out to investigate the relationship between blood measurements and recent life changes. No significant correlations were obtained.

\subsubsection{Relationship between Levels of Distress and Coping Across Time}

Rape is a stressor that disrupts homeostasis and requires adaptation, coping and/or defence mechanism. It is important to investigate the relationship between different types of distress that a rape survivor experiences and how one employs different efforts of coping. To ascertain whether there are any significant relationship between different Levels of Distress and Ways of Coping, across time 1, time 2 and time 3, Pearson product moment correlations were calculated.

The results obtained for time 1 where sample size $\mathrm{n}=12$ and $\mathrm{n}=5$, reveal no significant correlations. The results obtained for time 2 and time 3 are presented in tables 16 and 17 .

Table 16: Relationship between Levels of distress and Coping Time $2, n=5$

\begin{tabular}{|l|l|l|l|}
\hline Levels of Distress & Coping Scale & r & p \\
\hline phobic anxiety & emotional withholding & -0.932 & 0.021 \\
\hline phobic anxiety & wishful thinking & -0.942 & 0.017 \\
\hline interpersonal sensitivity & emotional withholding & -0.833 & 0.039 \\
\hline interpersonal sensitivity & problem focussed & -0.870 & 0.024 \\
\hline impact of event-intrusion & emotional withholding & 0.862 & 0.027 \\
\hline impact of event-intrusion & acceptance & 0.909 & 0.012 \\
\hline impact of event-intrusion & problem-focussed & 0.827 & 0.042 \\
\hline somatization & growth & -0.819 & 0.046 \\
\hline anxiety & growth & -0.872 & 0.024 \\
\hline obsessive & help-seeking & -0.950 & 0.013 \\
\hline psychotism & help-seeking & -0.923 & 0.009 \\
\hline
\end{tabular}


Table 17: Relationship between Levels of distress and Coping. Time $3, n=5$

\begin{tabular}{|l|l|l|l|}
\hline Levels of Distress & Coping Scale & $\mathbf{r}$ & $\mathbf{p}$ \\
\hline avoidance & emotion & 0.928 & 0.023 \\
\hline hostility & help-seeking & -0.996 & 0.040 \\
\hline
\end{tabular}

The results from Tables 16 and 17 are explained below.

\subsubsection{Relationship between levels of distress and the acceptance scale}

At time 2 , there is a significant positive correlation between the Intrusion Scale and the Acceptance Scale (see Table 16).

\subsubsection{Relationship between levels of distress and the emotional withholding scale}

There is a significant negative correlation between the Phobic Anxiety Scale and the Emotional Withholding Scale at time 2 ( see Table 16). At time 2 significant negative correlations also exist between the Interpersonal Sensitivity Scale and the Emotional Withholding Scale, as well as between the Intrusion Scale and the Emotional Withholding Scale. At time 3, a significant positive correlation is evident between the Avoidance Scale and the Emotional Withholding Scale (see Table 17).

\subsubsection{Relationship between levels of distress and the wishful thinking scale}

A significant negative correlation exists between the Phobic Anxiety Scale and the Wishful Thinking Scale at time 2, as indicated in Table 16.

\subsubsection{Relationship between levels of distress and the growth scale}

At time 3 significant negative correlations exist between the Somatization Scale and the Growth Scale, as well as between the Anxiety Scale and the Growth Scale (see Table 17).

\subsubsection{Relationship between levels of distress and the problem solving / help-seeking scale} The Interpersonal Sensitivity Scale and the Problem-focused/Help-Seeking scale have a significant negative correlation at time 2 (see Table 16). Whereas the Intrusion Scale and the Problem- 
focused / Help-seeking scale at time 2 have a significant positive correlation (see Table 16).

\subsubsection{Relationship between levels of distress and the help-seeking scale}

At time 2, a significant negative correlation exists between the Obsessive-Compulsive Scale and the Help-seeking Scale (see Table 16). Table 16 also indicates a significant negative correlation between the Psychotism Scale and the Help-seeking Scale at time 2. At time 3 a significant negative correlation is noted between the Hostility Scale and the Help-seeking Scale (see Table 17).

\subsubsection{Conclusion}

It is evident that both emotion focused ways of coping and problem focused ways of coping have significant relationships with various scales of distress as indicated above. 


\section{DISCUSSION}

\subsection{Introduction}

This study embarked on investigating the relationship between the impact of rape, coping efforts, health profile and immune parameters across an acute stress period of 35 days in rape survivors. This study also aimed to investigate what happens across time with respect to the immune parameters, levels of distress, ways of coping and health profile, after rape. In general this study provides some interesting results. In considering the results of the present study it is important to note the risk of including so many variables for such a small sample size. This is problematic because of the large number of correlation coefficients derived from the many comparisons of variables could arise purely by chance and it would be impossible to decide on which to reject and which to accept. Furthermore a normal distribution cannot be guaranteed. The following discussion of these results is structured around the demographic factors and the hypotheses of this study. The first hypothesis concerns the relationship between the levels of distress and immune parameters. The second hypothesis focuses on the relationship between the ways of coping and immune parameters. The third hypothesis pertains to the relationship between the rape survivors' health profile and immune parameters. Although the relationship between the levels of distress and ways of coping was not part of the original hypotheses, it will be discussed.

\subsection{Demographic Details}

The demographic details reveal that the rape survivors in this study are characterized as being young, predominantly Zulu speaking, single, unemployed women who have completed at least a high school standard of education. This is probably not representative of the overall profile of people being raped. Only a small percentage of all rapes are included in this research. The statistics on rape reveal that approximately 1 in 35 rapes are reported, (PACSA, 1998). Furthermore the sites of data collection were situated closely to rural areas, where the population is predominantly African, Zulu speaking people with a low socio-economic status. Whether or not a greater percentage of African, Zulu speaking, unemployed, single women with a relatively low level of education are at risk of getting raped needs to be explored. The rape survivors who participated in this study stem from a low socio-economic environment, are dependent on the state medical services for treatment and would not be able to afford private medical and psychological 
treatment.

The responses obtained in this study might have a cultural bias, since $92 \%$ of the participants were African and Zulu speaking. The results also indicate that $42 \%$ of the participants were HIV positive prior to the rape. This raises some concern about the participants well-being, especially in light of the traumatic, stressful experience of rape. HIV infection and other opportunistic infections might progress more rapidly under stress because the immune system is suppressed (Glaser et al.,1994)

\section{Individual Participants Across Time}

The summary of the psychological and immune measures for each participant across time reveals no dramatic changes across time. There are noticeable differences between the HIV negative participants and the HIV positive participants. These differences are observed indicate variability in the responses by the HIV positive participants to the psychological measures across time, as well as suppressed immunity due to the low CD 4 levels and raised White Cell Count levels. The low CD 4 count is expected due to these participants being HIV positive.

\subsection{The relationship between the levels of distress and immune parameters}

The hypothesis which states that there is a significant relationship between levels of distress and immune parameters was confirmed. This is in accordance with what is suggested in the literature (Glaser et al.,1994; Maier, Watkins \& Fleshner, 1994; Rabin, 1999). The levels of distress across time will be discussed initially. This is followed by a discussion of the immune parameters across time. Thereafter the relationship between the levels of distress and immune parameters would be discussed.

\subsubsection{The Levels of Distress Across Time}

Even though the Friedman tests revealed no significant differences in the levels of distress across time, there are interesting trends in the levels of distress (measured by the Brief Symptom Inventory and the Impact of Events Scale) across time. The levels of distress are immediate psychological and social reactions to rape which may last for days or weeks and are usually quite severe (McFarlance, 1995). Donaldson (1997) suggests that the symptoms of distress are 
manifested in various degrees and dimensions.

With the exception of the Obsessive-Compulsive sub-scale, all the other sub-scales of the Brief Symptom Inventory and the Impact of Events Scale indicate an increase in the mean scores from time 1 to time 2, that is from day 5 to day 15. This means that there is an increase in the amount of distress reported by rape survivors from time 1 to time 2 . This is followed by a decrease in the mean scores from time 2 to time 3, that is from day 15 to day 35 (see Figures 3 and 4). This means that there is a decrease in the amount of distress reported by rape survivors from time 2 to time 3.This is interesting since a higher level of distress is expected soon after the rape (at time 1), rather than 15 days after the rape (at time 2). However time 2 coincided with the post-test counselling for HIV and the feedback of the baseline HIV antibody test results to the rape survivors. This might have contributed to the higher level of distress obtained at time 2 . However the data collection at time 2 was prior to the post-test counselling. This was done to alleviate any effects of the post-test counselling. In between time 1 and time 2, rape survivors probably have to deal with family members, partners, police investigations, the fear of contracting HIV and sexually transmitted diseases, their own thoughts and feelings about the rape and any other consequences of the rape. These factors might also contribute to the heightened level of distress at time 2. The literature suggests that even the most homogenous of stress conditions is likely to elicit a wide range of responses resulting from genetic differences in physiologic reactivity, to coping and personality style and to learned responses (Keller et al., 1994). Rape, like other traumatic experiences, can be regarded as a life change event, which can be very distressing. The rape survivor will have to reassess her belief system, trust in people, explore issues around her sexuality. There are also many precipitating and predisposing factors such as previous life experiences, belief and value systems and rape myths which could also contribute to the heightened level of distress.

The Obsessive-Compulsive Scale is the only scale which does not resemble the trend displayed by the other level of distress scales. The Obsessive-Compulsive Scale is consistent at time 1 and time 2. This means that this level of distress remains the same up to 15 days after the rape. Rape survivors experience many unremitting and unwanted thoughts, impulses and actions as indicated by the symptom measured by this scale. The symptoms include trouble remembering things, having 
to check and double check what you do and difficulty making decisions. These symptoms are all characteristic of the symptoms experienced by rape survivors after rape. The decrease in all levels of distress from time 2 to time 3 might be due to the post-test counselling. Also during this period a decrease in levels of distress is expected because the rape survivor should be employing more appropriate coping mechanisms in an attempt to bring about a level of equilibrium.

These results suggest that rape survivors need more support and psychological / counselling intervention at time 2 than at time 1 and time 3 . This finding has major implications for the current services provided to rape survivors. At present the rape survivors who choose to report the incident, go through a routine medical examination by a district surgeon to confirm whether the survivor was raped or not. This is followed by pre-test HIV counselling and sometimes prescription of medication to prevent pregnancy and sometimes painkillers and antibiotics are prescribed or recommended. This service is presently provided by some government clinics and hospitals. No rape counselling is routinely provided. Non-government organisations like Rape Crisis complement government services by offering crisis intervention and counselling. The results obtained with regard to the levels of distress, suggest that rape survivors would benefit from counselling at least 15 days after the rape because levels of distress are higher at this point. HIV / AIDS appears to be a 'secondary trauma' to rape, which often becomes a primary concern for the rape survivor. This is based on the researchers experience from working with rape survivors over the part few years. Perhaps as a routine rape counselling should incorporate the pre and post test counselling for HIV. Therefore the counsellors need to be appropriately trained to carry out this task.

\subsubsection{The Immune Parameters Across Time}

The results illustrated in Figures $3 \mathrm{a}, \mathrm{b}, \mathrm{c}, \mathrm{g}, \mathrm{h}, \mathrm{l}$ and o indicate that there is a decrease in the mean level of $\mathrm{CD} 3 \%, \mathrm{CD} 3$ absolute, $\mathrm{CD} 4 \%$, neutrophil \%, neutrophil absolute, monocyte absolute and white cell count from time 1 to time 2 . The mean levels of CD $3 \%, \operatorname{CD} 3$ absolute, CD $4 \%$, neutrophil $\%$, neutrophil absolute, monocytes absolute and white cell count indicate an increase from time 2 to time 3 . The mean level of $\mathrm{CD} 8 \%, \mathrm{CD} 8$ absolute and monocyte $\%$ increases across time 1,2 and 3 . 
Literature on post stress immune changes reveals mixed results. A study by Bachen et al., (1992) looked at brief experimental stress, where the Stroop test was administered for 21 minutes. This study found a decrease in $\mathrm{CD} 4 \%$, but no changes in $\mathrm{CD} 3$ and B cells. A study carried out by Crary et al., (cited in Kiecolt-Glaser et al., 1992) also revealed a decreased \% of CD 4 cells subsequent to short-term stressors. The other immune parameters investigated in this study were not considered in the study by Bachen et al., (1992). Further research in recommended to investigate the increases and decreases in these immune parameters. An increase in CD 8 levels was also obtained in research carried out by Brosschot, Benschop, Godaert, Olff, DeSmet, Heijnen and Ballieux (1992). The American Psychosomatic Society (1992) report that CD 8 numbers seemed to increase in studies with longer and / or more intensive stressors. Therefore the results obtained for the $\mathrm{CD} 8$ levels are consistent with the literature findings even though the stress was a natural traumatic event and very different from the experimental stressors measured in other studies. The results obtained in this study differs from the results obtained for other naturalistic stressor like examination stress, which indicates a decrease or no effect on the number of CD 8 lymphocytes. These differences in findings might be due to the very different characteristics of the stressors being measured.

The results indicate an increase in the levels of lymphocytes and eosinophils from time 1 to time 2. This is followed by a decrease in these cell levels from time 2 to time 3 . The results also indicate that both $\mathrm{CD} 4$ absolute and platelets decrease across time. There is a significant difference in the $\mathrm{CD} 4 \%$ between time 1 and time 2 and a significant difference between time 2 and time 3 . A significant difference is also note for the platelets counts between time 1 and 2 and time 2 and 3 .

The increases and decreases in the mean levels of the immune parameters could be due to various factors. These include the circadian rhythms, menstrual cycle, new cells being manufactured and released into the blood flow, stress and behaviour for example appetite and sleep changes ( Keller et al., 1994 ). These authors also note that diumal variations in immunological parameters are well documented. During the data collection of this study, attempts were made to obtain psychological and blood measures at a fixed time to avoid variations in data collection. Keller et al. ( 1994 ) also suggest that measured alterations in immunity may be as a result of a) The occurrence and duration of mediating physiologic responses in relation to the stressor / distress; b) the time 
required for the immune system to react to the mediating factors and c) The time required for the altered immune processes to return to baseline. Because of the correlational design of this study , it is not possible to determine causation. Therefore whether the stress of rape causes these alterations or whether other factors do, needs to be investigated in further research. The literature in psychoneuroimmunology suggests that immune parameters return to a resting level in approximately one hour following cessation of the stressor ( Kiecol-Glaser et al., 1992). Whether or not this factor contributes to the variation in the cell numbers across time could not be ascertained in this study. Further research is required to address this.

The significant difference in the CD $4 \%$ and platelets exists even though all the CD $4 \%$ and platelet values are within the normal range as specified by the laboratory. While this might appear confusing, this stems from the fact that the immune function indicators may vary within a fairly wide range of 'normal' values or extend outside the normal range (Rabin, 1999). Keller et al., (1994) state that the relationship of such changes, within or exceeding the normative ranges, to health consequences is not necessarily predictable. Immune changes within the 'normal' range may still be associated with health changes, however large samples are probably required to detect such effects. This is not possible with this study due to the small sample size. The monocyte \%, is slightly out of the normal range $(0.48 \%)$. Health consequences with regard to this increase cannot be predicted in this study. Further research is required to address this.

\subsubsection{The relationship between Levels of Distress and Immune Parameters across time}

Significant relationships exist between levels of distress and immune parameters as indicated in Tables 4,5,6 and 7, presented in section 6 . The data obtained from the immune measures is a count of the cells, thus providing quantitative information about the cells present. The functional ability of these cell types cannot be determined. Speculations about the implications of the significant relationships are offered. The significant positive correlations between levels of distress and immune parameters suggests that an increase in a specific measure of distress can be associated with an augmented immune response. The strength of the immune response depends on the number and effectiveness of functional cells. Conversely a significant negative correlation suggests that an increase in a specific measure of distress can be associated with a decreased immune response. 
A literature search by the author revealed that no other research in psychoneuroimmunology, to date has looked at the relationship between the levels of distress ( measured by the Brief Symptom Inventory and the Impact of Events scale) and cell mediated immunity, subsequent to rape However there is evidence (Cohen \& Herbert, 1996; Glaser \& Kiecolt-Glaser, 1994; KiecoltGlaser et al. 1992; Pennebaker et al., 1988) suggesting a strong relationship between other stressors, for example examination stress, marital stress, laboratory stress and the immune system and in many instances these relationships have been causal. The literature reveals that the most consistent immune changes subsequent to acute stressor exposure is an increased Natural Killer cell and suppressor / cytotoxic $\mathrm{T}$ cell number and decreased proliferative response to mitogens, particularly phytohaemaglutinin. (Herbert et al., 1994; Manuck et al., 1991; Naliboff et al., 1991; Zakowski et al., 1992).. These results were obtained from studies on acute stressors lasting 15 20 minutes in the laboratory. The characteristics of those stressors are very different from that of rape, in that rape involves threat, loss of control, violence and violation to the survivor. Furthermore Kiecolt-Glaser,et al., (1992) suggest that immune parameters return to a resting level in approximately one hour following cessation of the stressor. This could not be ascertained in this study. Most studies look at pre and post test immune changes, this was impossible in this study.

The results presented in Table 4,5,6 and 7 (see section 6) indicate that various scales of distress have significant positive and negative relationships with various immune measures at time 1,2 and 3. One possible interpretation for this might be that rape may cause multiple distress effects, and the multiple distress effects caused by stressors might have a relationship with the immune parameters either independently, additively or interactively (Glaser et al., 1994). The pathway of these relationships need to be investigated.

The significant correlations between the levels of distress and the immune parameters at time 1 , time 2 and time 3 (see Table 4,5,6 and 7, section 6 ) might be due to direct or indirect mediating mechanisms between the experience of distress due to the rape and the immune parameters. The literature in psychoneuroimmunology reveals that there are a number of underlying central nervous system mediated physiological systems involved in mediating between stress / distress and immune functioning (Blalock et al., 1985, cited in Maier et al., $1994 \&$ Rabin, 1999). These systems include, but are not limited to endocrine, autonomic and peptidergic processes, for example 
positive and negative emotions have distinct patterns of autonomic nervous system responses, (Glaser et al., 1994 ). The autonomic nervous system has been shown to innervate immune organs such as the thymus, the lymph nodes and the spleen ( Maier et al., 1994; Pike et al., 1997). Furthermore neurotransmitter effects on lymphocytes are well documented (Blalock et al., 1985)

In addition to the central nervous system mechanisms that mediate stress immune processes, a variety of behaviours may indirectly effect immune functioning. These include routine health related behaviours, such as physical exercise and acute or short-term reactions to stress, such as increased smoking. Alcohol consumption, drug use or abuse, poor diet and altered sleep (Kusaka, Kondou, and Morimoto,1992). These health-risk behaviours may arise in response to rape. These behaviours might cause indirect effects which include alterations in nutrition, liver functioning and stress reactions. Appetite disturbances can affect nutrition and consequently impact on cellmediated immunity, phagocyte function, complement system integrity and mucosal immunity (Keller et al., 1994). Investigating the various mediating mechanisms would certainly shed some light and provide a greater understanding of the significant correlations obtained between the levels of distress and the immune parameters. The information provided by this study is only correlational and does not provide a causal chain, therefore the meaning of these significant relationships cannot be determined. Subsequent research would need to focus on assessing why these significant relationships exist.

The results also indicate a time lapse between the levels of distress and the immune parameters for example interpersonal sensitivity at time 1 has a significant negative relationship with CD 8 absolute, measured at time 2 (see Table 6 and 7 for more significant correlations of this nature). No previous research appears to be done on the relationship between levels of distress experienced by rape survivors and the immune system. These significant findings might be due to interactions between the central nervous system, endocrine system and behaviour. These results suggests that the immune response is not immediate. This might be due to a cascade of events that occur in the central nervous system, the endocrine system and behaviour, before any immune reaction is noticed. Furthermore feedback mechanisms between the central nervous system, endocrine and immune system might also play a role in these significant relationships. This investigation is worth repeating on a larger sample size. 


\subsection{The Relationship between Ways of Coping and Immune Parameters}

\subsubsection{Introduction}

Traumatic situations, like rape seem particularly important to research because they have the power to transform individual and family lives, almost always drain personal resources and have potential impact on well-being and adjustment (Mikulincer et al.,1996). When a person experiences a traumatic event attempts are made to master the demands by using various coping strategies. This section initially looks at a description and explanation of the ways of coping across time. Thereafter a discussion of the relationship between the ways of coping and immune parameters will be presented.

\section{7,5.2 The Ways of Coping Across Time}

There are no significant differences in the Ways of Coping across time. However, it is worth discussing the trends in the coping efforts across time. The problem-focused ways of coping (that is, efforts to improve the troubled person-environment relationship by bringing about change) measured by the Problem-focused / Help-Seeking Scale and the Help-seeking Scale, decreased across time, as indicated in Figure $6 \mathrm{~b}$ and $6 \mathrm{f}$ (presented in section 6 ). This suggests that rape survivors attempts to seek information, advice and solve problems are greater soon after the rape and as time passes problem-focused efforts at coping are used less. Before and at time 1 rape survivors need information, and need to make decision about having an HIV test, seeking medical assistance, following through with police and legal procedures, deciding whether to inform and seek support from family members and friends about the rape.

The Acceptance Scale is the only emotion focused way of coping that decreases across time. This suggests that at time 1 rape survivors might aim to minimize the negative impact of the stress once it has emerged, by either selectively ignoring what has happened, substituting activities, having patience, using bargaining or compromising strategies. Aldwin (1994) suggests that coping strategies most commonly used dealing with rape are bargaining, freeing oneself and alerting others, which might be seeking social support or reporting rape to the relevant authorities. The decrease in the use of problem-focused ways of coping and emotion-focused ways of coping measured by the Acceptance Scale could also be because as time passes the reality of the rape and 
the consequences might be more difficult to accept.

The other emotion-focused ways of coping presented a different picture across time. The SelfBlame Scale increases across time, suggesting that across the 35 day period the participants cope with stress by blaming or criticizing themselves for being unable to deal with the situation. This is a normal and expected reaction subsequent to rape (Allison \& Wrightsman, 1993). The Emotional Withholding Scale is highest at time 3. This is possibly due to rape survivors trying to avoid showing vulnerability or dependency feelings 35 days after the rape. This is probably an attempt to regain some control over the rape situation and their lives. The increase from time 1 to time 2 on the Growth Scale could possibly be due to rape survivors making efforts to buffer the stressful impact of the rape by controlling the meaning of the rape. There also appears to be a greater opportunity for personal growth inherent in the rape situation soon after the rape, that is at time 1 and time 2. This might be because rape survivors feel some control after reporting the rape and receiving some assistance after the rape. Tedeschi \& Calhoun (1995) suggest that when traumas are addressed through confrontation with them, constructive living and psychological growth are possible. People can adopt an attitude toward their suffering that allows it to cease to be mere suffering. Instead, traumatic events can be made to have meaning and be an opportunity for growth (Averill \& Nunley, 1992). It is interesting to note that the Growth Scale decreases at time 3. The Wishful Thinking / Escape Scale, which measures cognitive efforts to escape from emotional discomfort, indicates that at time 2 this coping effort is higher than that measured at time 1 and time 3. This might be due to an increase in emotion-focused strategies aimed at emotional escape by means of fantasy, humour and wishful thinking. The anticipation of the posttest counselling might also contribute to this raised measurement at time 2 . The decrease in the use of coping strategies measured by the Wishful Thinking / Escape Scale might be because other ways of coping like Emotional Withholding and Self-Blame become more dominant. The results indicate an increase in these ways of coping at time 3.

It is apparent from this study that neither one type of coping was used exclusively, but rather complex combinations of problem-focused and emotion-focused methods are used to cope with the rape. This is according to what is suggested in the literature (Monat et al.,1991). 


\subsubsection{The relationship between Ways of Coping and Immune Parameters across time}

The hypothesis which states that there is a significant relationship between ways of coping and the immune parameters was confirmed. No previous literature appears to be done on the relationship between a rape survivors ways of coping and immune parameters. The results obtained certainly provides valuable information for further research. Studies of social support as a coping strategy reveal that high, positive social support can serve as a buffer against the effects of a stressor on immunity or can even be associated with an augmented immune response. Conversely, low, negative social support can enhance the deleterious effects of a stressor on immunity ( Glaser, Kiecolt-Glaser, Stout, Tarr, Speicher \& Holliday, 1985; Glaser et al. 1994 ).

Monat and Lazarus (1991) suggest that coping methods depend upon the conditions being faced, the options available and possibly the persons' personality styles. With regard to this study some of the conditions faced by the rape survivor varied by the type of rape (that is whether it was gang rape, date rape, stranger rape and marital rape); the social support available; how much of violence was used; whether they had been raped previously, availability of medical attention, the choice to report the rape or not and who to disclose the rape to. Therefore these conditions and options together with the rape survivors personality might determine how they cope with the rape.

At times 1,2 and 3, the coping styles measured by various scales have significant relationships with various immune parameters. The reader is referred to Tables $8,9,10$ and 11 (section 6) for a presentation of the significant relationships. Since this data obtained for the immune measures is a measure of the cell count and not of the functional ability of the cells, conclusions about the functional ability of the immune system cannot be made. However speculations about the implications of the significant relationships are offered. The significant positive correlations between ways of coping and immune parameters suggests that an increase in a specific measure of coping can be associated with an augmented immune response. A coping strategy can be seen as buffering the distress. The strength of the immune response depends on the number and effectiveness of functional cells. Conversely a significant negative correlation suggests that an increase in a specific measure of coping style can be associated with a decreased immune response. This could be because a particular way of coping is not successful in buffering the level of distress. 
Coping efforts could impact on the levels of distress and thus through various mediating mechanisms produce changes to immune parameters. Immunity can be influenced by behavioural changes that occur as adaptations or coping responses in the face of stressful events or negative emotional states. For instance, people experiencing negative affect often engage in poor health practices, such as smoking, poor dietary practice or poor sleeping habits (Cohen \& Williamson, 1995). According to Kiecolt-Glaser \& Glaser (1988b ), this may have immunosuppresive effects.

The results also indicate a time delay in establishing significant relationships between ways of coping and immune parameters. These significant findings might be due to a cascade of interactions between the central nervous system, endocrine system and behaviour. Feedback mechanisms between the various body systems might also play a role in these significant relationships. No previous research could be found on the time delayed relationship between rape survivors ways of coping and immune parameters. It would be interesting to further investigate the pathways followed and the causes of these significant relationships.

Coping is a context-bound process and coping strategies that provide benefits in one situation may prove ineffective in another. Even within the same situation contextual, interpersonal and intrapsychic factors may alter the adequacy of attempts to cope with stressful events (Mikulincer et al., 1996 ). Inappropriate health behaviours like increased smoking and alcohol consumption might also indirectly contribute to the significant relationships between specific ways of coping and specific immune parameters. No definite conclusions can be drawn with regard to the causes of the significant correlations between the ways of coping and the immune parameters. Since no causal conclusions can be made, it is difficult to ascertain whether the coping efforts played a role in disease susceptibility or not. Further research needs to look at whether the coping efforts and immune parameters produce significant relationships independently, together or in combinations and to explore the causal relationships.

The knowledge gained from this study may provide a backdrop for theoretical and applied insight into the processes involved in successful and unsuccessful coping and its relationship with the immune system. 


\subsection{The relationship between the Duke-UNC Health Profile and the Immune Parameters}

\subsubsection{Introduction}

A rape survivor's health status can provide an indication of her overall well-being. Using the Duke-UNC Health Profile the health status can be measured along four dimension which are symptom status, physical function, emotional function and social function. This section initially discusses the rape survivors health profile across time. This is followed by a discussion of the relationship between the health profile and immune parameters.

\subsubsection{The Health Profile across time}

Although there are no significant differences in the scales of the health profile across time, it is worth discussing the differences across time 1,2 and 3. The results of the Symptom Status Scale indicate that there is an increase in symptoms being reported across time. Since symptoms are a natural expression of dysfunction within the body and mind (Parkerson et al., 1981), the results suggest that over a 35 day period the amount of dysfunction reported increases. This means that across a 35 day period the rape survivors general health status decreases. It is important to note that these symptoms might often be vague and non-specific because the same symptom can result from a variety of physical or psychological disorders. The Social Function Scale follows a different trend to the Symptom Status Scale, in that there is an increase in the rape survivors' ability to function in the workplace or at home, interact with people and participate in community and social events. It is expected that rape survivors should be able to carry out these social activities within this time period. The Emotional Function Scale indicates an increase in a rape survivors selfesteem, from time 1 to time 2 , and a decrease from time 2 to time 3 . The increase in self-esteem is possibly due to rape survivors feeling more in control, feeling better about themselves after reporting the rape and getting the necessary medical attention. The decrease in the Emotional Function Scale at time 2,15 days after the rape, might be due to $42 \%$ of the participants finding out that they were already HIV positive prior to the rape. All the participants had no idea of their HIV status at the beginning of the study, therefore the news of their HIV status could possibly have some effect on their emotional functioning and self esteem. Further research is needed to investigate this. The Physical Status Scale follows a similar trend to the Emotional Function Scale in that there is an increase from time 1 to time 2 and a decrease from time 2 to time 3 . This 
suggests that rape survivor's perceived capacity to perform tasks increases between time 1 and time 2. The decrease in the perceived capacity to perform activities is interesting and unexpected, because at 35 days after the rape, a rape survivor is expected to have less disability days, better ambulation and better upper extremity function. This decrease in physical function is worth investigating in further research.

With regard to the rape survivors' overall well-being the results indicate that up to 15 days after the rape there is an increase in reporting of symptoms on all four dimensions of the health profile. There is an increase in the Symptom Status dimension and the Social Function dimension from time 2 to time 3 . At this same time there is a decrease in symptoms reported on the Emotional Function and the Physical Status dimension. Therefore there is improvement in certain aspects of the rape survivors' health profile only.

\subsubsection{The relationship between the Duke-UNC health Profile and Immune Parameters}

Hypothesis 3, which states that there is a relationship between the rape survivors' health profile and the immune system has been confirmed with respect to the significant relationships between the Symptom Status, Emotional Function and Social Function dimensions and specific immune parameters. The results presented in Table 12,13,14 and 15 (section 6) show significant correlations between the Symptom Status Scale, the Social Status Scale and the Emotional Function Scale, and specific immune parameters. No significant relationship was evident between the Physical Function Scale and any immune parameter. The significant negative correlations suggests that a decrease in reporting of symptoms on the respective health profile dimensions is associated with an increase in the specific immune parameters. It is important to note that if a specific number of cells were present, it does not mean that all does cells are capable of functioning. There might be some native cells or cells in different stages of maturity (Rabin, 1999). If these cell populations are functional it could be speculated that a decrease in the specific dimensions of the health profile would be associated with enhancement of the cell-mediated immunity. A significant positive correlation suggests that an increase in the dimensions of the health profile is associated with an increase in the number of cells of the specific cell populations. Therefore if these cells are functional, it can be speculated that an increase in specific dimensions of the health profile is associated with immune enhancement. 
The sample size was reduced from $n=12$ to $n=5$ to facilitate statistical analysis across time 1,2 and 3. The significant relationships between the health profile scale and the immune parameters at time $1(n=12)$ and time $1(n=5)$ are very different, in that at time $1(n=12)$ the Symptom Status Scale and Social Status Scale have significant negative correlations with white cell count, and the Social Status Scale has a significant negative correlation with neutrophil absolute, and at time $1(n=5)$ a significant positive correlation exists between the Emotional Function Scale and CD $4 \%$ and a significant negative correlation is evident between the Emotional Function Scale and white cell count. The change in sample size might contribute to these differences in significant relationships. This analysis is worth repeating on a larger sample size.

The significant relationships already outlined in Tables $12,13,14$ and 15 might be due to various mediating effects of the central nervous system, and the endocrine system as well as indirect effects. The significant relationships between the Symptom Status Scale, measured at time 2 and monocyte \% and eosinophil \%, measured at time 3, might be due to some delayed feedback response. It is expected that a rape survivors overall health status is associated with immune function. Therefore a better health status is associated with immune enhancement and the poorer the health status is, the more compromised the immune system would be. The design of the research does not allow determination of the causes of the significant relationships. This is worth exploring in further research.

\section{The relationship between the Levels of Distress and Ways of Coping}

Rape results in the survivor experiencing various levels of distress and symptoms which are collectively referred to as rape trauma syndrome (McFarlance,1995; Rape Crisis Manual, 1998; 1999). The responses of the rape survivor are not abnormal. The distress they experience is a normal response to an extremely traumatic and abnormal event, over which they have little control. Subsequent to a stressful event a person becomes aware of psychophysiological responses. If those responses are interpreted as potentially dangerous and disturbing, the person may then decide to employ one or many response management, or coping techniques (Rahe, 1995). The coping activities are consciously directed behaviours intended to modify and to terminate the psychophysiological responses. Investigators in the field of stress and coping rely primarily on cross-sectional and occasional coping assessments that require participants to recall a stressful 
encounter and then reconstruct how they responded to it. Research is now moving towards a process approach (Tennen, Affleck, Armeli \& Carney, 2000). This study follows current trend in coping research in that it allowed for tracking rapidly fluctuating processes such as coping close to the real-time of occurrence. This might provide unique insights into conceptually and clinically challenging questions in this field of study.

Although no hypothesis was originally stated about the relationship between the levels of distress and ways of coping it is worth investigating. The results reveal that a significant relationship exists between specific levels of distress scales and specific ways of coping scales only at time 2 and time 3.The results indicate that there are no significant relationships between specific levels of distress and specific ways of coping at time 1 . This might be because at time 1 the rape survivor might be experiencing a sense of overwhelming shock, confusion or a sense of unreality due to the violent nature of the trauma so might need to learn and adopt new methods of coping.. Significant relationships between specific levels of distress and specific ways of coping are noted at time 2 and time 3. At time 2 the Emotional Withholding Scale and the Wishful -thinking Scale have a significant negative correlation with the Phobic Anxiety Scale. This suggests that rape survivors who report a higher usage of emotional withholding and wishful thinking, report less phobic anxiety. This means that Emotional Withholding and Wishful-thinking might buffer the symptoms of Phobic Anxiety. The Emotional Withholding Scale and the Problem Focused / Help-seeking Scale both have a significant negative correlation with the Interpersonal Sensitivity Scale. This means that Emotional Withholding and Problem-focused-help-seeking methods of coping might be effective in buffering the distress described by the Interpersonal Sensitivity Scale. The Intrusion Scale has a significant positive correlation with the Emotional Withholding Scale, the Acceptance Scale and the Problem -Focused / Helpseeking Scale. A rape survivor experiences many intrusive thoughts (for example flashbacks ) and feelings (for example fear). This means that 15 days after the rape, rape survivors experience intrusive thoughts and feelings that are coped with by using Emotional Withholding, Acceptance and Problem-Focused / Help-seeking methods. Both the Somatization Scale and the Anxiety Scale have a significant negative correlation with the Growth Scale. This suggests that growth after the traumatic rape experience can be achieved if somatization and anxiety are reduced. The Obsessive Compulsive Scale and the Psychotism Scale both have significant negative correlations with the Help-seeking Scale. This means that rape 
survivors who adopt a withdrawn, isolated, schizoid life style and who have thoughts, impulses and actions that are experienced as unwanted and irresistible are less likely to seek help and vice versa. This can have long term consequences for rape survivors especially with regard to social interactions.

The significant relationship at time 3 presents a different picture. A significant positive correlation between the Emotional Withholding Scale and the Avoidance Scale exists. There are rape survivors who carry on life as normal, and tend to block out the feelings and thoughts about the rape. This is a normal reaction to rape and reveals attempts that the rape survivor makes to try and lead as normal a life as possible through avoidance and emotional withholding. A significant negative correlation between the Help-seeking Scale and the Hostility Scale exists. Rape survivors might have a generalized anger and disillusionment with the people with whom she might have contact and the world in general, this can be associated with them seeking less assistance. The differences in the significant relationships across time suggests that coping is a fluctuating process, however the cause of these significant relationships is worth exploring in further research and with a larger sample size.

Overall this study indicates that there is a significant relationship between the distress experienced after rape, ways of coping, health profile and cell-mediated immune parameters across time. Furthermore there are significant differences in the CD $4 \%$ and platelet count across time. The knowledge gained from this study certainly provides a foundation for further research in psychoneuroimmunology focussing particularly on natural acute traumatic stressors like rape. Even though no causal conclusion can be ascertained, this study certainly contributes to the exploding field of PNI and strengthens the literature by indicating the significant relationships between levels of distress, ways of coping, health profile and immune parameters do exist. This can form the foundation for further research. 


\section{8 Implications of the research}

The findings of the study have important implications in the following ways. The results can aid in understanding and developing appropriate intervention strategies, that can be implemented soon after the rape. An interesting finding from this study indicates that distress is highest at time 2 (15 days after the rape). Perhaps counselling and psychotherapeutic services could be routinely provided at this time to all rape survivors. Furthermore in many government clinics in South Africa routine post-HIV test counselling is done at this time, perhaps rape counselling can be incorporated with HIV counselling. The counsellors would need to be appropriately trained. At the time of reporting the rape, rape survivors should be given the necessary information about the physical, emotional, cognitive and behavioural reactions to rape, so that they are prepared and not extremely distressed when they find themselves thinking, feeling or behaving in certain ways. This might also reduce the level of distress that rape survivors experience after the rape.

The findings of this research can provide relevant information to facilitate the development of more appropriate health care policies. Health care policies can include rape counselling. The results indicate that $42 \%$ of the sample are already HIV positive prior to the rape. This can have implications for health care provision after rape. Stress plays a role in compromising immune system functioning, therefore HIV positive rape survivors are at a greater risk of becoming symptomatic or being infected by other HIV strains if the perpetrator was HIV positive. Health care policies need to include mental health care as well as medical health care.

The results suggest that central nervous system, endocrine and immune system are associated with each other through various pathways. This highlights immunity as a link between the mind and body

The knowledge gained provides theoretical and applied insight into the processes involved subsequent to a traumatic rape experience. The significant relationships between levels of distress, ways of coping, health profile and immune parameters contributes to the theoretical understanding of these processes and provides information about a rape survivors' experiences. 


\subsection{Limitations of the Research}

A brief discussion will be presented around the methodological and conceptual problems inherent in this study. Recommendations for further research would also be suggested.

This study is restricted to self-report and peripheral blood measurements, which involve relatively non-invasive techniques. These are amenable to repeated measures and do not require specific sensitization. However the ability to fully assess psychoneuroimmunological processes is restricted, measures of processes in the spleen, lymph nodes, skin and thymus are excluded. These body regions play an integral role in the psychoneuroimmunological processes. Investigations of the immune processes in the spleen, lymph nodes, skin and thymus would certainly give some insight to these processes. However PNI research on humans do not include such invasive techniques. This might also pose some ethical and cost dilemmas.

A major limitation to this study is the small sample size. The small sample size does not allow for more elaborate statistical analysis. The small sample size could be because data collection occurred at an extremely traumatic period in the participant's life, and many were not ready to participate in the research five days after the rape. Furthermore not all people report the rape within five days of the rape. There was a high drop out and lack of follow up. This was probably because participants had other priorities or had difficulties in getting transport to the hospital by a certain time for data collection. Many lived in rural areas and depended on public transport. Many participants could not afford transport. When money was provided by the researcher to cover transport costs, the money was often misappropriated. In an attempt to obtain accurate results, the blood samples were collected at the same time on the specific days, this also contributed to the small sample size. If the participants arrived late or on a different date, their data was not included in the analysis. This study only targeted rape survivors who choose to report the rape, therefore the sample is not representative of the population. A larger sample size could be obtained by collecting data throughout South Africa and trying to include those rape survivors who do not report the rape but obtain medical and psychological assistance. This study is worth repeating on a larger sample size. 
The study was based on a single group correlational design. The correlational design does not allow for any causal conclusions. A baseline measurement prior to the rape is impossible to obtain, therefore it is difficult to comment on a persons' immune state before the rape.

The repetition of the psychometric questions over the three instances might of caused contamination of responses on times 2 and 3 by earlier responses. This should be investigated in further studies.

The cost of the blood testing determined the type of testing, only cell mediated immunity was assessed. More elaborate tests for example tests measuring mediating factors , humoral immunity and viral load would certainly provide more information about the overall interactions with the immune system.

All the self-report instruments used in this study have not been validated in South Africa. Many of the tests have been used with subjects whose home language is not English, however they have not been standardised on a Zulu speaking population. Another limitation pertains to the translation of the instruments into Zulu. Although much care was taken to preserve the original meaning of all the test items, some items were difficult to translate and even when translated were ambiguous. It is recommended that these self-report instruments be validated in South Africa or other selfreport measured be designed which might be more appropriate to the South African population.

Other recommendations for further research

- A study designed to investigate the causes or pathways of the significant relationships'

- $\quad$ Research investigating the differences between HIV positive rape survivors and HIV negative rape survivors

- Research investigating other aspects of the immune system like humoral immunity might provide more information about immune responses. Further research needs to investigate 
the functional capability of various cell populations so that conclusions can be made about functioning of the immune system.

- A longitudinal study looking at long-term disease susceptibility might provide information about a rape survivors' health outcome subsequent to rape. Longitudinal studies might also provide information about the relationship between levels of distress, ways of coping, health profile and immune parameters soon after the rape and more long term consequences with regard to levels of distress, ways of coping, health profile and immune parameters.

- A study investigating cross cultural issues inherent in levels of distress experienced following rape, ways of coping and health profile might provide valuable information which could be used for designing appropriate intervention strategies. 


\section{REFERENCES}

Ader, R. \& Cohen, N. (1993). Psychoneuroimmunology : Conditioning and Stress. Annual Review of Psychology. 44, 53 - 85.

Adler, N. \& Matthews, K. (1994). Health Psychology: Why do some people get sick and some stay well? Annual Review of Psychology. 45, 229 - 259.

Aldwin, C.M. (1994). Stress, Coping and Development- An Integrative Perspective. The Guilford Press. London.

Allison, J.A. \& Wrighstman, L.S.(1993). Rape -The Misunderstood Crime. SAGE publications. London.

American Psychiatric Association (1994). Diagnostic and Statistical Manual of Mental Disorders ( $4^{\text {th }}$ ed.) Washington,DC.

Averill, J.R. \& Nunley, E.P. (1992). Voyages of the Health- Living on Emotionally Creative Life. New York : Free Press.

Bachen, E.A., Manuck, S.B., Marsland, A.L., Cohen, S., Malkoff, S.B., Muldoon, M.F.\& Rabin, B.S. (1992). Lymphocyte Subset and Cellular Immune Response to a Brief Experimental Stressor. Psychosomatic Medicine 54, 673 - 679.

Baum, A., Cohen, L. \& Hall, M. (1993). Control \& Intrusive Memories as Possible Determinants of Chronic Stress. Psychosomatic Medicine. 55, 274 -286.

Blalock, J.E., Smith, E.M. \& Meyer, W.J. (1985) in Maier, S.F; Watkins, L.R. \& Fleshner, M. (1994). Psychoneuroimmunology - The Interface Between Brain, Behaviour and Immunity. American Psychologist: 49 (12), 1004 - 1017. 
Boyce, W.T., Chesney, M., Alkon, A., Tschann, J.M., Adams, S., Chesterman, B., Cohen, F., Kaiser, P., Folkman, S. \& Wara, D. (1995). Psychobiologic Reactivity to Stress and Childhood Respiratory Illness : Results of two prospective studies. Psychosomatic Medicine, 57, 411 - 422.

Brosschot, J.F., Benschop, R.J., Godaert, G.L.R., Olff, M., DeSmet, M., Heijen, C.J.\& Ballieux, R.E. (1994). Influence of Life Stress on Immunological Reactivity to Mild Psychological Stress. Psychosomatic Medicine, 56, 216 - 224.

Burgess, A.W., \& Holmstrom, L.L. (1974). Rape Trauma Syndrome. American Journal of Psychiatry $131,981-999$.

Calhoun,K.S. \& Atkeson,B.M. (1991).Treatment of Rape Victims: Facilitating Psychosocial Adjustment. New York: Pergamon.

Cohen, S., Doyle, W.J., Skoner, D.P., Fireman, P.,Gwaltney, J.M. \& Newson, J.T. (1995), State and Trait Negative Affect as Predictors of Objective and Subjective Symptoms of Respiratory Viral Infections. Journal of personality and Social Psychology. 68 (1), 159 - 169.

Cohen, S. \& Herbert, T.B. (1996). Health Psychology: Psychological Factors and Physical Disease from the Perspective of Human Psychoneuroimmunology. Annual Review of Psychology. 47, 113 -142 .

Cohen, S. \& Manuck, S.B. (1995). Stress, Reactivity and Disease. Psychosomatic Medicine. $57,423-430$.

Cohen, S., Tyrrell, D.A.J. \& Smith, A.P. (1993). Negative Life Events, Perceived Stress and Negative Affect and Susceptibility to the Common Cold. Journal of Personality and Social Psychology. 64, $131-140$.

Cohen, S. \& Williamson, G.M. (1991). Stress and Infectious Disease in Humans, Psychological Bulletin. 109 (1), 5 - 24. 
Cohen, S. \& Williamson, G.M. (1995). Perceived Stress in a Probability Sample of the United States. In S. Spacapan and S. Oskamp (Eds.) The Social Psychology of Health. Nceobury CA : Sage.

Coopersith, S.(1967). The Antecedents of Self-Esteem. San Francisco: WH Freeman and Company

Deogatis, L.R., \& Spencer, R.M. (1982). The Brief Symptom Inventory (BSI) Administration and Procedures: BSI - manual -1. USA : Clinical Psychometric Research.

Donaldson, S. (1997). Rape Trauma Syndrome in male prisoners President.Stop Prisoner rape, Inc. http://www.igc. apc.org/spr/docs/rts.html.

Dunn, A.J. (1992). Endotoxin-induced Activation of Cerebral Catecholamine and Serotonin Metabolism: Comparison with interleukin-1. Journal of Pharmacology \& Experimental Therapeutics, 261, $964-969$.

Dunn, A.J. (1995). Psychoneuroimmunology: Introduction and General Perspectives In Leonard, B.E. \& Miller, K. (Eds.) Stress, the Immune system and Psychiatry. New York: John Wiley and Sons.

Eagle, A.S.(1987). Sex, Sex-role Orientation and the Self-Report of Coping Preferences: An exploratory study. Unpublished masters thesis. University of Natal - Pietermartizburg

Esterling, B.A., Antoni,M.H., Kumar,M.\& Schneiderman, N. (1990). Emotional Repression, Stress Disclosure Responses and Epstein-Barr Viral Capsid Antigen Titers. Psychosomatic Medicine, 52, $397-410$.

Esterling, B.A., Kiecolt-Glaser, J.K.; Bodnar, J.C. \& Glaser, R. (1994). Chronic Stress, Social Support, and Persistent Alterations in the Natural Killer Cell Response to Cytokines in Older Adults. Health Psychology. 13, 291 - 298. 
Fawzy, I.\& Fawzy, M.D. (1995). Behaviour and Immunity. In Kaplan, H.I. and Saddock, B.J.(eds.) Comprehensive Textbook of Psychiatry $4^{\text {th }}$ Ed. (2). Tokyo:Williams and Wilkins.

Felten, D.L., Cohen, N., Ader, R., Felten, S., Carlson, S \& Roszman, T. (1991). Central Neural Circuits Involved in Neural-Immune Interactions. In Ader, R., Felten, D.L. \& Cohen, N. (Eds.) Psychoneuroimmunolgy. $2^{\text {nd }}$ Ed.Boston: Academic Press, Inc.

Felten, D.L., Felten, S.Y., Carlson, S.L., Olschowka, J.A. \& Livnat, S ( 1985). Noradrenergic sympathetic innervation of lymphoid tissue. Journal of Immunology. $135,755 \mathrm{~s}-765 \mathrm{~s}$.

Felten, S.Y. \& Felten, D.L. (1991). Innervation of lymphoid tissue. In R. Ader; D.L. Felten \& N. Cohen (Eds) Psychoneuroimmunology $2^{\text {nd }}$ ed. San Diego: Academic Press.

Folkman, S. \& Lazarus, R.S. (1980). An analysis of coping in a middle-aged community sample. Journal of Health and Social Behaviour. 21, 219 - 239.

Glaser et al. 1982 in Stone, A.A.; Cox, D.S.; Valdimarsdottir, H.; Jandorf, L. \& Neale, J.M. (1987). Evidence that Secretory IgA antibody is associated with Daily Mood. Journal of Personality and Social Psychology. 52 (5), 988 - 993.

Glaser, R. \& Kiecolt-Glaser, J.K. ( 1994) . Hand Book of Human Stress and Immunity. London:Academic Press.

Herbert, T.B. \& Cohen, S. (1993). Depression and Immunity: A meta-analytic review. Psychological Bulletin, 113, $472-486$.

Herbert, T.B., Cohen, S., Marsland, A.L., Bachen, E.A., Rabin, B.S., Muldoon, M.F. \& Manuck, S.B. (1994). Cardiovascular reactivity and the course of immune response to an acute psychological stressor. Psychosomatic Medicine. 56, 337 - 344.

Hodgkinson, P.E. \& Stewart,M.(1991). Coping with Catastrophe. London: Routledge 
Holroyd, K.A \& Lazarus, R.S. (1982). Stress, coping and somatic adaptation. In L. Goldberger \& S. Breznitz (Eds.), Handbook of stress: Theoretical and clinical aspects. New York.: The Free Press.

Horowitz, M:; Nancy, W.\& Alvarez, W. (1979). Impact of Event Scale: A measure of subjective stress. Psychosomatic Medicine. 41 (3), 209-218.

Irwin, M., Brown,M., Patterson, T., Hauger,R., Mascovich, A \& Grant,I. (1991). Neuropeptide-y and Natural Killer Cell Activity: Findings in Depression and Alzheimer Caregiver Stress. FASEB I $5,3100-3100$.

Keller, S.E., Shiflett, S.C., Schleifer, S.J. \& Bartlett, J.A. (1994). Stress, Immunity and Health.. In R. Glaser \& Kiecolt-Glaser, J.K. ( 1994) . Hand book of human stress and immunity. London: Academic Press.

Kemeny, M.E., Cohen, F., Zegans, L.S. \& Conant, M.A. (1989). Psychological and Immunological Predictors of Genital Herpes Recurrence. Psychosomatic Medicine, 51, 195 - 208.

Kiecolt-Glaser, J.K., Cacioppo, J.T., Malarkey, W.B. \& Glaser, R. (1992)._Psychosomatic Medicine. 54, $680-685$.

Kiecolt-Glaser, J.K., Dura, J.R, Speicher, C.E., Trask, J.\& Glaser, R. (1991) Spousal Caregivers of Dementia Victims: Longitudinal Changes in Immunity and Health. Psychosomatic Medicine. $53,345-362$.

Kiecolt-Glaser, J.K. \& Glaser, R. ( 1987). Psychological Influences on Herpes Virus Latency. In E. Kurstak; Z.J. Lipowski \& P.V. Morozor (eds.) Viruses, Immunity \& Mental Disorders. New York Plenum Press, $403-412$.

Kiecolt-Glaser, J.K. \& Glaser, R. (1988a). Methodological Issues in Behavioural Immunological Research with Humans. Brain behaviour and Immunity, 2, 67-68. 
Kiecolt-Glaser, J.K.\& Glaser, R. ( 1988b). Psychological Influences on Immunity- Implications for AIDS. American Psychologist. 43(11), 892 - 898.

Kiecolt-Glaser, J.K. \& Glaser, R. ( 1995). Psychoneuroimmunology \& Health Consequences : Data \& Shared Mechanisms. Psychosomatic Medicine. 57, 269 - 274.

Kiecolt-Glaser, J.K., Glaser, R., Shuttleworth, E.C., Dyer,C.S., Ogrocki, P. \& Speicher, E.C (1987). Chronic Stress and Immunity in Family Caregivers of Alzheimer's Disease Victims. Psychosomatic Medicine. 49, 523 - 535.

Kilpatrick, D.G., Vernen L.J. \& Best, C.L.(1985). Factors Predicting Psychological Distress Among Rape Victims. In C.R. Figley (Ed), Trauma and its wake: The study and treatment of posttraumatic stress disorder, voll. New York: Brunner

Kusaka, Y., Kondou, H. \& Morimoto, K., (1992). Healthy Lifestyles are Associated wit Higher Natural Killer Activity. Preventive Medicine. 21, 602 - 615

Lazarus, R.S., DeLongis, A, Folkman, S. \& Gruen, R (1985). Stress and Adaptational Outcomes The Problem of Confounded Measures. American Psychologist, 40 (7), 770 - 779.

Lazarus, R.S. \& Folkman, S. (1984). Stress, Appraisal and Coping. New York: Springer Company.

Leonard, B.E. \& Miller, K. (1995 ).Stress, The Immune System and Psychiatry. Toronto: John Wiley and Sons.

Lewis, S. (1994) Dealing with Rape. Johannesburg: SACHED Books.

Levy,S.M., Fernstorm, J., Herberman, R.B., Whiteside, T., Lee, J., Ward, M. \& Massoudi, M. (1991). Persistently Low Natural Killer Activity and Circulating Levels of Plasma beta endorphin Risk Factors for Infectious Disease. Life Science, 48, 107 - 116. 
Linn, B.S., Linn, M.W. \& Jensen, J. (1981) Anxiety and Immune Responsiveness. Psychological Reports. $49,969-970$.

Linthorst, A.C.E., Flachskamm, C., Holsboer, F. \& Reul, J.M.H.M. (1994). Local Administration of Recombinant Human interleukin-16 in the Rat Hippocampus Increases Serotonergic Neurotransmitter, Hypothalamic-pituitary-adrenocortical Axis Activity and Body Temperature. Endocrinology, 135, 520-532.

Maier, S.F., Watkins, L.R. \& Fleshner, M. (1994). Psychoneuroimmunology. The Interface between behaviour, Brain \& Immunity. American Psychologist 49(12), 1004 - 1017.

Maier, S.F. \& Watkins, L.R. (1998). Cytokines for Psychologists: Implications of Bidirectional Immune to Brain communication for understanding behaviour, mood and cognition. Psychological Review, 105 (1) 83 - 107.

Manuck, S.B., Cohen, S., Rabin, B.S., Muldoon, M.F. \& Bachen, E.A. (1991).Individual Differences in Cellular Immune Response to Stress. Psychological Science 2, $111-115$.

Marsland, A.L., Manuck, S.B., Fezzari, T.V., Steward, C.J. \& Rabin, B.S. (1995). Stability of Individual Differences in Cellular Immune Responses to Acute Psychological Stress. Psychosomatic Medicine. 57, 295 - 298.

McFarlane, A.C. (1995). Stress and disaster. In S.E. Hobfa and M.W. deVries (Eds.), Extreme Stress and Communities. The Netherlands : Kluwer.

McCann, I.L., Sakheim, D.K. \& Abrahamson, D.J.(1988). Trauma and Victimization: A Model of Psychological Adaptation. Counselling Psychologist, 16,531 - 594.

McClelland, D.C., Alexander, C. \& Marks, E. (1982). The Need for Power, Stress, Immune Function and Illness Among Male Prisoners. Journal of Abnormal Psychology, 91, 61 - 70. 
McKinnon, W., Weisse, C.S., Reynolds, C.P., Bowles, C.A. \& Baum, A (1991) Chronic Stress, Leukocytes Subpopulation and Humoral Response to Latent Viruses. Health Psychology 8, 389 402 .

Monat, A.\& Lazarus, R.S. (1991). Stress and Coping - An Anthology $3^{\text {rd }}$ ed. New York: Columbia University Press.

Mikulincer, M. \& Florian, V. (1996). Coping and Adaptation to Trauma and Loss. In Zeidner,M. \& Endler, N.S. (Eds.) Handbook of Coping Theory, research and Application. New York: John Wiley and Sons.

Naliboff, B.D., Benton, D., Solomon, G.F., Morley, J.E. \& Fahey, J.L.(1991). Immunological Changes in Young and Old Adults During Brief Laboratory Stress. Psychosomatic Medicine 53, $121-132$.

Norusis, M.J. (1990). SPSSIPC + 4.0 Base Manual. Chicargo:SPSS Inc.

O'Leary, A. (1990). Stress, Emotion \& Human Immune Function. Psychological Bulletin. 108 (3), 363-382.

Ottaway, C.A. \& Husband, A.J. (1992). Central Nervous System Influences on Lymphocyte Migration. Brain, Behaviour and Immunity, 6, 97 - 116.

PACSA, (1998). Rape, factsheet , 44.

Parkinson, F. (1997). Critical Incident Debriefing - Understanding and Dealing with Trauma. Canada: Souvenir Press (E and A) Ltd.

Parkerson, G.R., Gehlbach, S.H., Wagner, E.H., James, S.A., Nancy, E.C. \& Muhlbaier, L.H. (1981). The Duke-UNC Health Profile: An Adult Health Status Instrument for Primary Care. Medical Care. XIX (8), 806 - 828. 
Pennebaker, J.W., Kiecolt-Glaser, J.K. \& Glaser, R. (1988). Disclosure of Traumas and Immune Function: Health Implications for Psychotherapy. Journal of Consulting and Clinical Psychology $56,239-245$.

Pike, J.K., Smith, T.L., Hauger, R.L., Nicassio, P.M., Patterson, T.L., McClintick, J., Costlow, C. \& Irwin, M.R. (1997). Chronic Life Stress Alter Sympathetic, Neuroendocrine and Immune Responsitivity to an Acute Psychological Stressor in Humans. Psychosomatic Medicine, 59, 447 457.

Pillay, P. (1999) unpublished paper on Sexual Violence and HIV/AIDS presented at the University of Natal - Pmb. Annual HIV/AIDS conference.

Rabin, B.S. (1999). Stress, Immune Function and Health - The Connection. New York: WileyLiss.

Rahe, R.H. (1995). Stress and Psychiatry. In Kaplan, H.I. and Saddock, B.J.(eds.) Comprehensive Textbook of Psychiatry VI ed (2). Tokyo: Williams and Wilkins.

Rape Crisis. (1998). Rape Crisis Training Manual. unpublished manual.

Rape Crisis. (1999) Rape Crisis Training Manual. unpublished manual.

Roszman, T.1. \& Carlson, S.l. (1991). Neurotransmitters and molecular signalling in the immune response. In R. Ader; N.Cohen \& D.L. Felten (Eds.). Psychoneuroimmunology, $2^{\text {nd }}$ ed. New York: Academic Press.

Roszman, T.L., Cross, R.J., Brooks, W.H. \& Markesbery, W.R. (1985). Neuroimmunomodulation: Effects of Neural Lesions on Cellular Immunity. In R. Guillemin, M. Cohn \& T. Melnechuk (Eds.). Neural modulation of immunity. New York: Raven Press. 
Sadock, V.A. (1995). Physical and Sexual Abuse of Adult. In Kaplan, H.I. and Saddock, B.J.(eds.) Comprehensive Textbook of Psychiatry VI ed (2). Tokyo:Williams and Wilkins.

Salk, J. (1962). Biological basis of disease and behaviour. Perspectives in Biology and Medicine, $5,198-206$.

Sarason, I.G., Sarason, B.R., Potter, E.H. \& Antoni, M.H. (1985). Life Events, Social Support and Illness. Psychosomatic Medicine, 47, 156-163.

Schleifer, S.J. \& Keller, S.E. (1991). Stress, Depression and the Immune System. Directions in Psychiatry. $11(16), 1-9$.

Schleifer, S.J., Keller, S.E., Bond, R.N., Cohen, J. \& Stein, M. (1989).Major Depressive Disorder and Immunity - Role of Age, Sex, Severity and Hospitalization. Archieves of General Psychiatry, $46,81-87$.

Sieber, W.J., Rodin, J., Larson, L., Ortega, S. \& Cummings, N. (1992). Modulation of Human Natural Killer Cell Activity by Exposure to Uncontrollable Stress. Brain, Behaviour and Immunity $6,141-156$.

Smith, A.P., Tyrrell, D.A.J., Al-Nakib, W., Coyle, K.B. \& Donovan, C.B. (1988). The Effects of Experimentally Induced Respiratory Virus Infections on Performance. Psychological Medicine. $18,65-71$.

Snyder,C.R.\& Ford,C.E. (1987). Coping with Negative Life Events - Clinical and Social Psychological Perspectives. New York: Plenum Press.

Solomon, G.F. (1990) The Re-Emerging Field of Psychoneuroimmunology, with a Special Note on ADS. In Ornstein, R \& Swencionis (Eds.) ( 1990 ). The Healing Brain - A Scientific Reader. New York: The Guildford Press. 
Solomon, G.F.(1995). Immune and Nervous System Interactions An analytic bibliography supporting key postulates on communication links, similarities and implications. California: The Fund for Psychoneuroimmunology.

Stone, A.A., Cox, D.S., Valdimarsdottir, H., Jandorf, L. \& Neale, J.M. (1987). Evidence that Secretory IgA Antibody is Associated with Daily Mood.Journal of Personality and Social Psychology. 52 (5), 988 - 993.

Stone, A., Porter, L., \& Neale, J.M. (1993). Daily Events and Mood Prior to the Onset of Respiratory Illness Episodes. A Non-Replication of the 3-5 day 'Desirability Dip'. British Journal of Medical Psychology, 66, 383 - 393.

Stone, A.A., Reed, B.R. \& Neale, J.M. (1987). Changes in Daily Event Frequency Precede Episodes of Physical Symptoms. Journal of Human Stress, 13, 70 - 74.

Stone, A.A, Valdimarsdottir, H.B., Katkin, E.S., Burns, J., Cox, D. S., Lee, S., Fine, J., Ingle, D \& Bovbjerg, D.H. (1993). Effects of Mental Stressors on Mitogen-Induced Lymphocyte Responses in the Laboratory. Psychology and Health. 8, 269 - 284.

Suls, J. \& Fletcher, B. (1985). The Relative Efficacy of Avoidant and Non-avoidant Coping Strategies :A Meta Analysis. Health Psychology, 4, 247-288.

Tedeschi, R.G. \& Calhoun, L.G. (1995). Trauma and Transformation - Growing in the Aftermath of Suffering. London: SAGE Publications, Inc.

Tennen, H., Affleck, G., Armeli, S \& Carney, A. (2000). A Daily Process Approach to Coping Linking Theory, Research and Practise. American Psychologist, 55 ( 6 ), 626 - 636.

Tizard, I.R.(1995). Immunology an introduction $-4^{\text {th }}$ ed. Philadelphia: Saunders College Publishing. 
Turner-Cobb, J.M. \& Steptoe, A. (1996). Psychological Stress and Susceptibility to Upper Respiratory Tract Illness in an Adult Population Sample.Psychosomatic Medicine, 58, 404 - 412..

Van der Wal J. (1990). The Aftermath of Suicide :A Review of Empirical Evidence. Omega, 20, 149-171.

Vingerhoets, A.J.J.M., \& Flohr, P.J.M. (1984).Type A Behaviour and Self-Report of Coping Preferences. British Journal of Medical Psychology. 57, 15 - 21.

Zakowski, S.G., McAllister, C.G., Deal, M. \& Baum, A. (1992). Stress, Reactivity and Immune Function in Healthy Men. Health Psychology 11, 223 - 232.

Zeller, J.M. (1996) Psychoneuroimmunology: an Emerging Framework for Nursing Research. Journal of Advanced Nursing. 23, 657 - 664. 


\section{APPENDICES}

$-119-$ 
Appendix 1 - The Immune system 


\section{THE IMMUNE SYSTEM}

The following section provides an overview of the different immunological parameters, their function as a component and the reasons for measuring these various immune parameters.

The immune system has two major tasks. It must protect individuals against infectious agents invading the body from outside, and at the same time, it must prevent the development of abnormal cells within the body.

There are two types of immune responses (Tizard, 1995). One, mediated by proteins called antibodies, is responsible for resistance to infectious agents found in body fluids. The other, mediated by cells called lymphocytes, is responsible for the destruction of abnormal cells. Such abnormal cells include cancer cells and virus infected cells.

This research focuses on cell mediated immunity, only cells involved in this process will be discussed. Figure 8 illustrates the cells of the immune system and there respective origins, to give the reader an idea of how the various cells function with respect to each other.

$\mathrm{T}$ - cells:

These are the lymphocytes involved in cell mediated immunity. The T-cells measured in this study are $\mathrm{CD} 3, \mathrm{CD} 4$ and $\mathrm{CD} 8$.

CD 3

These are required for signal transduction from the $\mathrm{T}$-cell receptor [T cell receptor ] molecule to an intracellular tyrosine kinase. Many reactions of the immune system operate through this type of receptor. Transduction is the conversion of an extracellular signal into a series of intracellular events

\section{4 T lymphocytes}

These cells that help B lymphocytes to produce antibody. They also release cytokines that attract phagocytic cells and contribute to the killing of the infected cells. CD $4+$ produces IFN-gamma. IFN-gamma activates macrophages and induces killing of bacteria the macrophages have ingested.

CD 8 T lymphocytes:

These are able to recognise when a tissue cell is infected because the tissue cell displays a portion of the infectious agent in a presentation molecule [ MHC 1] that is on the surface of the tissue cell. CD 8 lymphocytes kill the tissue cell.

White Blood Cells:

These cells are collectively referred to as 'leukocytes' comprised of neutrophils, eosinophils, basophils, monocytes and lymphocytes.

* Neutrophils are involved in phagocytosis of solid particles such as bacteria.

* Eosinophils help protect against infections by parasites eg worms, by releasing proteins that are toxic to the parasites.

* Basophils arise from bone marrow and circulate in the blood. They do not usually enter the tissue, they have granules that contain histamine. 
* Monocytes ingest foreign materials that get into the body. Infectious agents that are ingested by monocytes are often killed when the monocyte / macrophage is activated by a $\mathrm{T}$ lymphocyte.

\section{Platelets:}

Platelets are a haematological measure but play a role with regard to removal of bacteria from the body. Blood platelets can adhere to and engulf some bacteria. Bacteria attached to platelets can be removed from the circulation and destroyed by the cells of the mononuclearphagocytic system. Platelets assist to remove bacteria that may hide in clumps of aggregated platelets and hence be protected from circulating antibodies and complement and so survive. 
FIGURE 8: THE CELLS OF THE IMMUNE SYSTEM AND THEIR ORIGINS

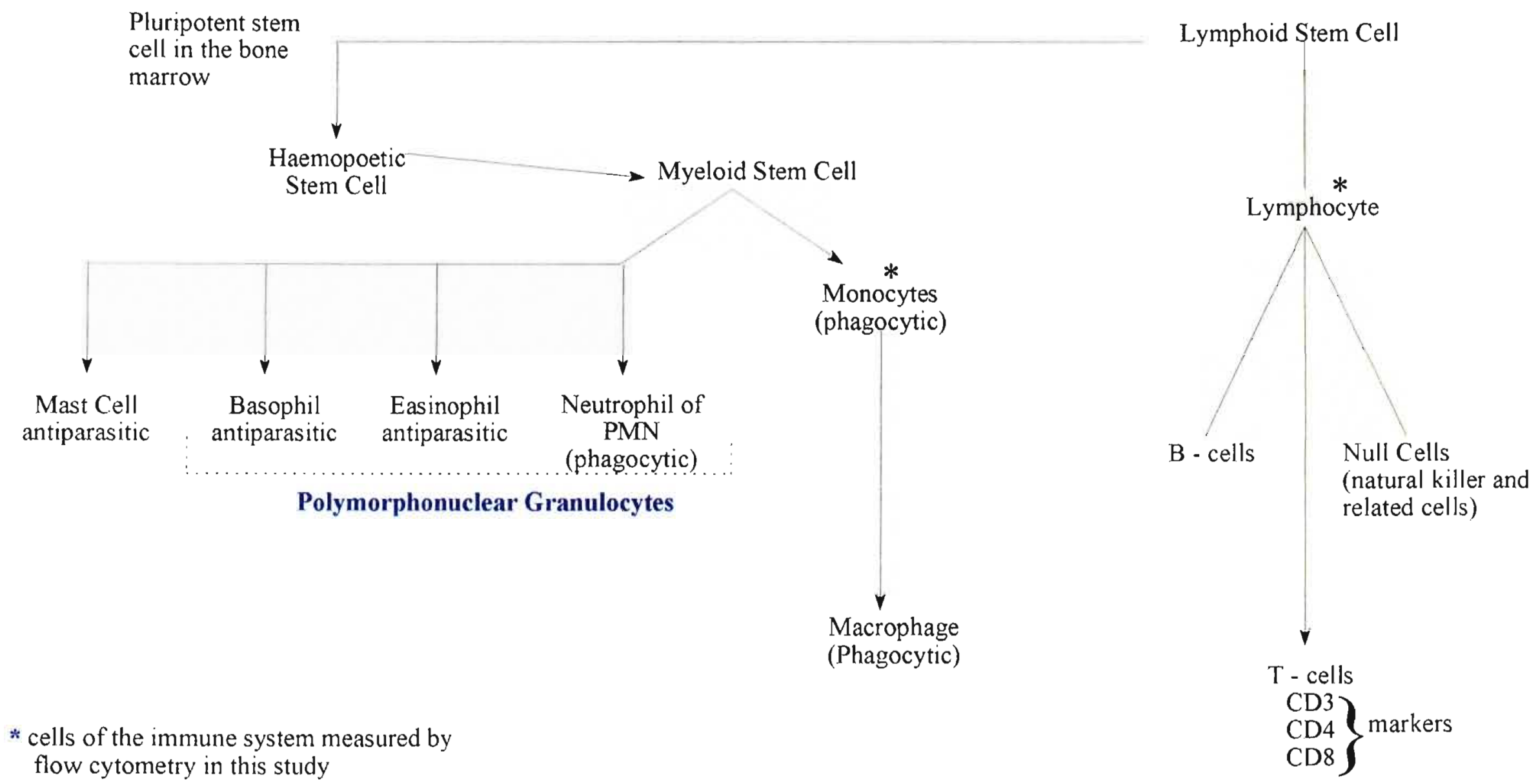


Appendix 2 - Questionnaires in English

Section 1: Brief Symptom Inventory

Section 2: Impact of Events Scale

Section 3: Recent Life Change Questionnaire

Section 4: Duke-UNC Health Profile

Section 5: Ways of Coping Checklist 


\section{CONFIDENTIAL QUESTIONNAIRE}

NB: You may refrain from answering specific questions if you so wish.

Date

Name

Home Address

Postal Address

Telephone number :

Home

Work

Cell

First Language:

English

Afrikaans

Zulu

Xhosa

Other

Sex:

Female

Male

Date of Birth:

Ethnic group:

Black

Coloured

Indian

Other

White

Religious affiliation:

Christian

Hindu

Muslim

Jewish

Marital Status:

\section{Single}

Married

Divorced

Separated

Current employment status:

Widowed

Employed part time

Employed full time Unemployed

Retired

What is the highest educational level that you have completed?

Less than high school

High school std completed

Technical or Business school

University

Other

When were you raped? 
Below is a list of problems people sometimes have. please read each one carefully, and circle the number to the right that best describes how much that problem has distressed or bothered you during the past 7 days including today. Circle only one number for each problem and do not skip any items. If you change your mind, erase your first mark carefully. Read the example below before beginning, and if you have any questions please ask about them.

\begin{tabular}{|c|c|c|c|c|c|}
\hline How much were you distressed by : & not at all & a little bit & moderately & quite a bit & extremely \\
\hline eg: bodyaches & 0 & 1 & 2 & 3 & 4 \\
\hline 1. Nervousness or shakiness inside & 0 & 1 & 2 & 3 & 4 \\
\hline 2. Faintness or dizziness & 0 & 1 & 2 & 3 & 4 \\
\hline 3. The idea that someone else can control your thoughts & 0 & 1 & 2 & 3 & 4 \\
\hline 4. Feeling that others are to blame for most of your troubles & 0 & 1 & 2 & 3 & 4 \\
\hline 5. Trouble remembering things & 0 & 1 & 2 & 3 & 4 \\
\hline 6. Feeling easily annoyed or irritated & 0 & 1 & 2 & 3 & 4 \\
\hline 7. Pains in heart or chest & 0 & 1 & 2 & 3 & 4 \\
\hline 8. Feeling afraid in open spaces & 0 & 1 & 2 & 3 & 4 \\
\hline 9. Thoughts of ending your life & 0 & 1 & 2 & 3 & 4 \\
\hline 10. Feeling that most people cannot be trusted & 0 & 1 & 2 & 3 & 4 \\
\hline 11. Poor appetite & 0 & 1 & 2 & 3 & 4 \\
\hline 12. Suddenly scared for no reason & 0 & 1 & 2 & 3 & 4 \\
\hline 13. Temper outburst that you could not control & 0 & 1 & 2 & 3 & 4 \\
\hline 14. Feeling lonely even when you are with people & 0 & 1 & 2 & 3 & 4 \\
\hline 15. Feeling blocked in getting things done & 0 & 1 & 2 & 3 & 4 \\
\hline 16. Feeling lonely & 0 & 1 & 2 & 3 & 4 \\
\hline 17. Feeling blue & 0 & 1 & 2 & 3 & 4 \\
\hline 18. Feeling no interest in things & 0 & 1 & 2 & 3 & 4 \\
\hline 19. Feeling fearful & 0 & 1 & 2 & 3 & 4 \\
\hline 20. Your feeling being easily hurt & 0 & 1 & 2 & 3 & 4 \\
\hline 21. Feeling that people are unfriendly or dislike you & 0 & 1 & 2 & 3 & 4. \\
\hline 22. Feeling inferior to others & 0 & 1 & 2 & 3 & 4 \\
\hline 23. Nausea or upset stomach & 0 & 1 & 2 & 3 & 4 \\
\hline 24. Feeling that you are watched or taked about by others & 0 & 1 & 2 & 3 & 4 \\
\hline 25. Trouble falling asleep & 0 & 1 & 2 & 3 & 4 \\
\hline 26. Having to check and double check what you do & 0 & 1 & 2 & 3 & 4 \\
\hline 27. Difficulty making decisions & 0 & 1 & 2 & 3 & 4 \\
\hline 28. Feeling afraid to travel on busses, subways or trains & 0 & 1 & 2 & 3 & 4 \\
\hline 29. Trouble getting your breath & 0 & 1 & 2 & 3 & 4 \\
\hline 30. Hot or cold spells & 0 & 1 & 2 & 3 & 4 \\
\hline $\begin{array}{l}\text { 31. Having to avoid certain things, places, or activities } \\
\text { because they frighten you }\end{array}$ & 0 & 1 & 2 & 3 & 4 \\
\hline 32. Your mind going blank & 0 & 1 & 2 & 3 & 4 \\
\hline 33. Numbness or tingling in parts of your body & 0 & 1 & 2 & 3 & 4 \\
\hline 34. The idea that you should be punished for all your sins & 0 & 1 & 2 & 3 & 4 \\
\hline
\end{tabular}




\begin{tabular}{|l|c|c|c|c|c|}
\hline How much were you distressed by : & not at all & a little bit & moderately & quite a bit & extremely \\
\hline 35. Feeling hopeless about the future & 0 & 1 & 2 & 3 & 4 \\
\hline 36. Trouble concentrating & 0 & 1 & 2 & 3 & 4 \\
\hline 37. Feeling weak in parts of your body & 0 & 1 & 2 & 3 & 4 \\
\hline 38. Feeling tense or keyed up & 0 & 1 & 2 & 3 & 4 \\
\hline 39. Thoughts of death or dying & 0 & 1 & 2 & 3 & 4 \\
\hline $\begin{array}{l}\text { 40. Having urges to beat, injure, or harm } \\
\text { someone }\end{array}$ & 0 & 1 & 2 & 3 & 4 \\
\hline 41. Having urges to break or smash things & 0 & 1 & 2 & 3 & 4 \\
\hline 42. Feeling very self-conscious with others & 0 & 1 & 2 & 3 & 4 \\
\hline 43. Feeling uneasy in crowds & 0 & 1 & 2 & 3 & 4 \\
\hline 44. Never feeling close to another person & 0 & 1 & 2 & 3 & 4 \\
\hline 45. Spells of terror or panic & 0 & 1 & 2 & 3 & 4 \\
\hline 46. Getting into frequent arguments & 0 & 1 & 2 & 3 & 4 \\
\hline 47. Feeling nervous when you are left alone & 0 & 1 & 2 & 3 & 4 \\
\hline $\begin{array}{l}\text { 48. Others not giving you proper credit for your } \\
\text { achievements }\end{array}$ & 0 & 1 & 2 & 3 & 4 \\
\hline 49. Feeling so restless you couldn't sit still & 0 & 1 & 2 & 3 & 4 \\
\hline 50. Feelings of worthlessness & 0 & 1 & 2 & 3 & 4 \\
\hline $\begin{array}{l}\text { 51. Feeling that people will take advantage of } \\
\text { you if you let them }\end{array}$ & 0 & 1 & 2 & 3 & 4 \\
\hline 52. Feelings of guilt & 0 & 1 & 2 & 3 & 4 \\
\hline $\begin{array}{l}\text { 53. The idea that something is wrong with your } \\
\text { mind }\end{array}$ & 0 & 1 & 2 & 3 & 4 \\
\hline
\end{tabular}

\section{SECTION 2}

Below is a list of comments made by people after stressful life events. Please check each item, indicating how frequently these comments were true for you DURING THE PAST SEVEN DAYS. If they did not occur during that time, please mark the "not at all" column.

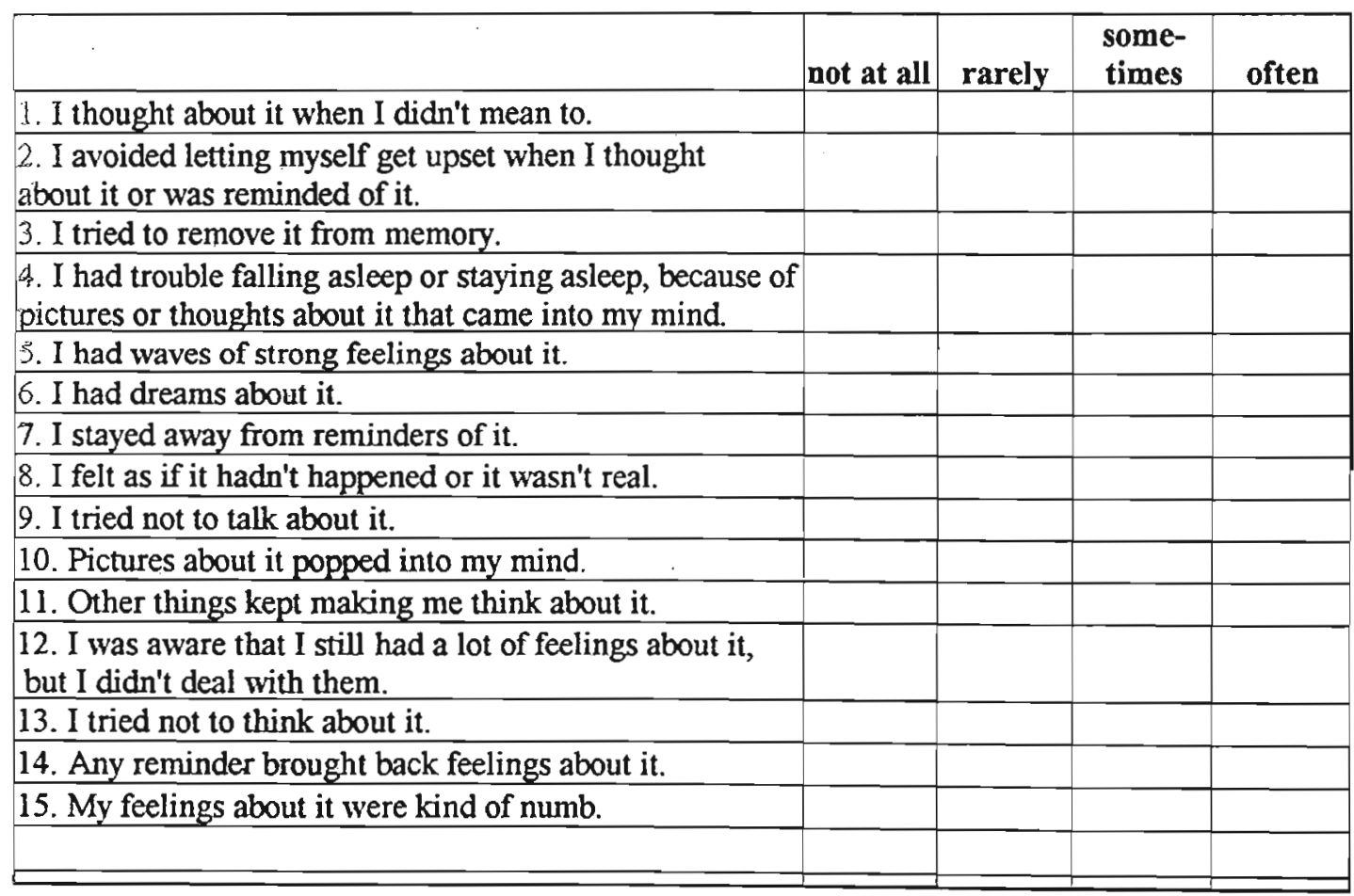




\section{SECTION 3}

To answer the questions below, marking one or more of the squares to the right of each question. If the event in question happened to you within the past two years, indicate when it occurred by marking in the appropriate column: 0-6 months ago, 7-12 months ago, etc. If you experienced an event more than once over the past two years, mark all appropriate squares. if the event did not occur over the last the last two years (or never occurred), leave all squares empty. Within the time periods listed, have you experienced?

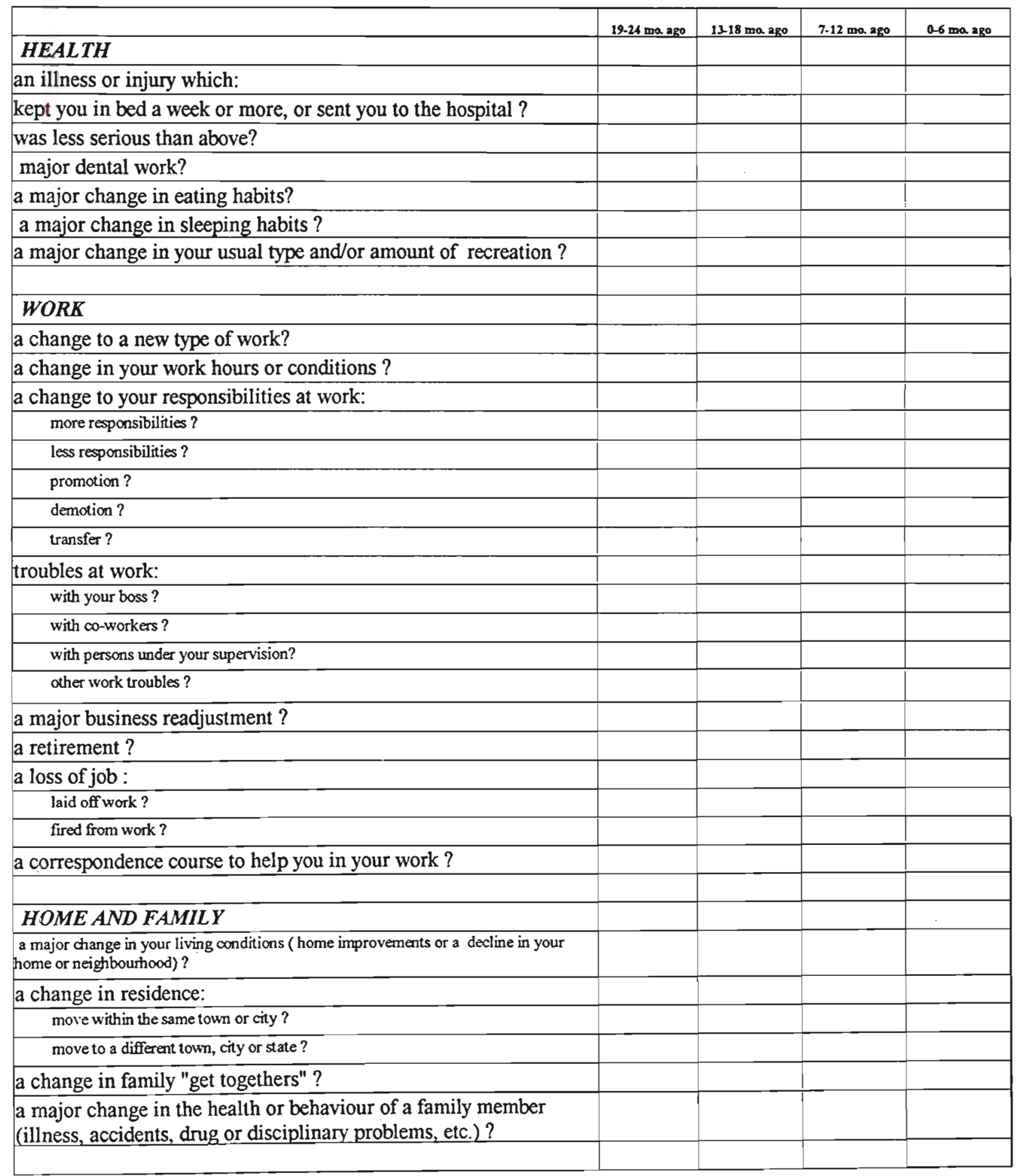




\begin{tabular}{|c|c|c|c|c|}
\hline & $19-24 \mathrm{ma} .230$ & $13-18 \mathrm{ma,} \mathrm{ago}$ & $7.12 \mathrm{ma} . \mathrm{ago}$ & $0,6 \operatorname{ma} .230$ \\
\hline \multicolumn{5}{|l|}{ marriage ? } \\
\hline \multicolumn{5}{|l|}{ a pregnancy? } \\
\hline \multicolumn{5}{|l|}{ a miscarriage or an abortion? } \\
\hline \multicolumn{5}{|l|}{ a gain of a new family member: } \\
\hline \multicolumn{5}{|l|}{ birth of a child? } \\
\hline \multicolumn{5}{|l|}{ adoption of a child? } \\
\hline \multicolumn{5}{|l|}{ a relative moving in with you? } \\
\hline \multicolumn{5}{|c|}{ a spouse beginning or ending work outside the home? } \\
\hline \multicolumn{5}{|l|}{ a child leaving home: } \\
\hline \multicolumn{5}{|l|}{ to attend college? } \\
\hline \multicolumn{5}{|l|}{ due to marriage? } \\
\hline \multicolumn{5}{|l|}{ for other reasons? } \\
\hline \multicolumn{5}{|c|}{ a change in arguments with your spouse? } \\
\hline \multicolumn{5}{|l|}{ in-law problems? } \\
\hline \multicolumn{5}{|c|}{ a change in marital status of your parents: } \\
\hline \multicolumn{5}{|l|}{ divorce? } \\
\hline \multicolumn{5}{|l|}{ remarriage? } \\
\hline \multicolumn{5}{|l|}{ a separation from your spouse: } \\
\hline \multicolumn{5}{|l|}{ due to work? } \\
\hline \multicolumn{5}{|l|}{ marital problems? } \\
\hline \multicolumn{5}{|l|}{ a divorce? } \\
\hline \multicolumn{5}{|l|}{ the birth of a grandchild? } \\
\hline \multicolumn{5}{|l|}{ the death of a spouse? } \\
\hline \multicolumn{5}{|l|}{ the death of another family member : } \\
\hline \multicolumn{5}{|l|}{ child? } \\
\hline \multicolumn{5}{|l|}{ brother or sister? } \\
\hline \multicolumn{5}{|l|}{ parent? } \\
\hline PERSONAL AND SOCLAL & & & & \\
\hline $\begin{array}{l}\text { a change in personal habits (your } \\
\text { etc? }\end{array}$ & & & & \\
\hline beginning or ending school or coll & & & & \\
\hline a change of school or college? & & & & \\
\hline a change in political beliefs? & & & & \\
\hline a change in religious beliefs? & & & & \\
\hline a change in social activities (clubs, & & & & \\
\hline a vacation? & & & & \\
\hline a new, close, personal relationship & & & & \\
\hline an engagement to marry? & & & & \\
\hline girlfriend or boyfriend problems ? & & & & \\
\hline
\end{tabular}




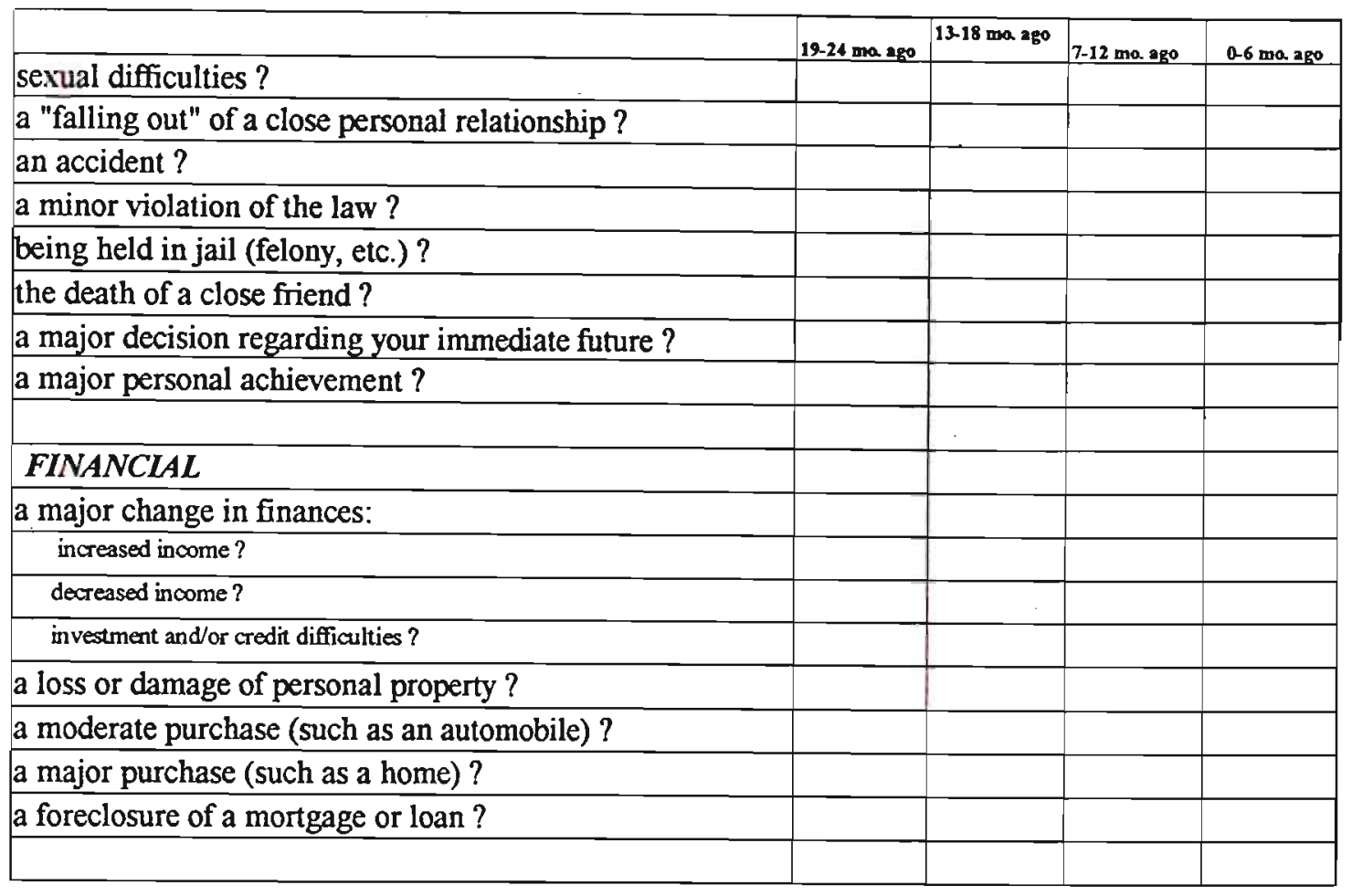

\section{SECTION 4}

Here are a number of questions about your health and feelings. Please read each question carefully and give your best answer. You should answer the questions in your own way. There are no right or wrong answers.

\begin{tabular}{|l|l|l|l|}
\hline During the past week : & & & \\
\hline How much trouble have you had with: & & & \\
\hline & NONE & SOME & A LOT \\
\hline 1. Eyesight & & & \\
\hline 2. Hearing & & & \\
\hline 3. Talking & & & \\
\hline 4. Smelling odours & & & \\
\hline 5. Tasting food & & & \\
\hline 6. Appetite & & & \\
\hline 7. Chewing & & & \\
\hline 8. Swallowing & & & \\
\hline 9. Moving your bowels & & & \\
\hline 10. Passing water / Urinating & & & \\
\hline 11. Breathing & & & \\
\hline 12. Sleeping & & & \\
\hline 13. Walking & & & \\
\hline 14. Headace & & & \\
\hline 15. Hurting or aching in any part ot your body. & & & \\
\hline 16. Itching in any part of your body. & & & \\
\hline
\end{tabular}




\begin{tabular}{|l|l|l|l|}
\hline & NONE & SOME & A LOT \\
\hline 17. Indigestion & & & \\
\hline 18. Fever & & & \\
\hline 19. Getting tired easily & & & \\
\hline 20. Fainting & & & \\
\hline 21. Poor memory & & & \\
\hline 22. Weakness in any part of your body & & & \\
\hline 23. Feeling depressed / sad & & & \\
\hline 24. Nervousness & & & \\
\hline $\begin{array}{l}\text { During the past month: } \\
\text { How much trouble have you had with: }\end{array}$ & & & \\
\hline 25. Weight loss & & & \\
\hline 26. Weight gain & & & \\
\hline 27. Unusual bleeding & & & \\
\hline 28. Sexual performance & & & \\
\hline
\end{tabular}

\section{During the past week:}

1. How many days were you in bed most of the day because of sickness, injury or health problems?

2. How many days did you stay in your $\overline{\text { none }} \quad \overline{1-4 \text { days }} \quad \overline{5-7 \text { days }}$ home because of sickness, injury or health problems?

3. How much trouble have you had getting around outside your home because of sickness, injury or health problems?

$\begin{array}{lll}\overline{\text { none }} & \overline{1-4 \text { days }} & \overline{5-7 \text { days }} \\ \overline{\text { none }} & \text { some } & \overline{\text { a lot }}\end{array}$

Today would you have any physical trouble or difficulty:

\begin{tabular}{|l|l|l|l|}
\hline & NONE & SOME & A LOT \\
\hline 4. Running 5 kilometers & & & \\
\hline 5. Holding a baby & & & \\
\hline 6. Walking up a flight of stairs & & & \\
\hline 7. Hearing the radio or TV & & & \\
\hline 8. Running a mile & & & \\
\hline 9. Walking the lenght of a football field. & & & \\
\hline 10. Walking to the bathroom & & & \\
\hline 11. Peeling an apple & & & \\
\hline 12. Reading a street sign & & & \\
\hline 13. running the lenght of a football field & & & \\
\hline 14. Reading a newspaper & & & \\
\hline 15. Combing your hair & & & \\
\hline
\end{tabular}


Here are some statements you could use to describe how you feel about yourself. Please read each statement carefully and place a $\mathbf{X}$ across the number that best fits how the statement describes you.

Here is an example:

I like T.V. soap operas.

$\begin{array}{llc}\text { Descrides } & \begin{array}{l}\text { Somewhat } \\ \text { describes me }\end{array} & \text { Doesn't describe } \\ \text { me exactly } & \text { me at all }\end{array}$

\begin{tabular}{lllll}
\hline 0 & $1 \mathrm{X}$ & 2 & 3 & 4
\end{tabular}

If you put an ' $\mathrm{X}$ ' as is marked, it means that liking T.V. soap operas describes you more than "somewhat" but not "exactly".

Answer each item as best as you can. Remember there are no right or wrong answers.

1. My body is in good shape for my age

2. I am a pleasant person

3. I don't feel useful

4. My friends look to me when they're in trouble

5. I get on well with members of the opposite sex

6. My family doesn't understand me

7. I like who I am

8. I feel hopeful about the future

9. I try to look my best

10. I am a clumsy person

11. I hate parties and social occasions

12. I have difficulty making decisions

13. I like meeting new people

14. I'm not an easy person to get along with

15. I'm a failure at everything I try to do

16. I'm basically a healthy person

17. I wish I had more sex appeal

18. I give up too easily

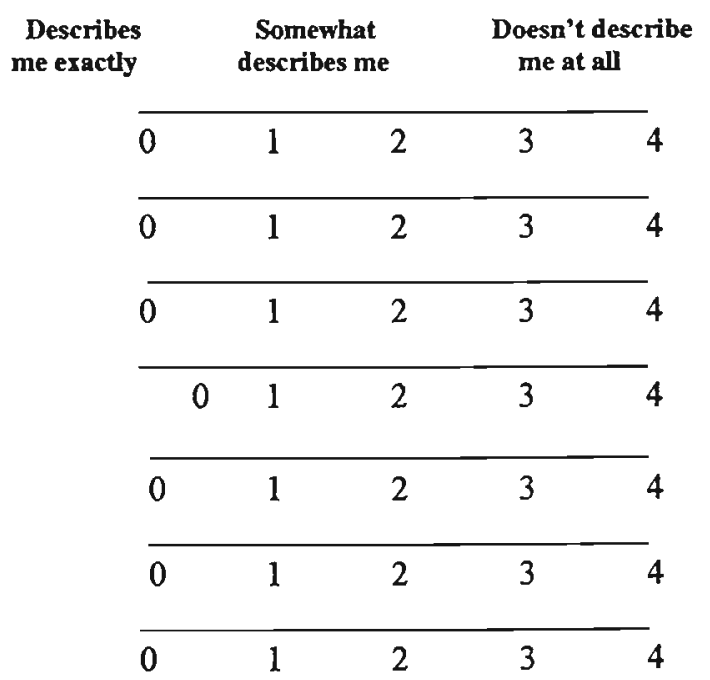

\begin{tabular}{llll}
\hline 0 & 1 & 2 & 3
\end{tabular}

\begin{tabular}{llll}
\hline 0 & 1 & 2 & 3
\end{tabular}

$\begin{array}{lllll}0 & 1 & 2 & 3 & 4\end{array}$

\begin{tabular}{lllll}
\hline 0 & 1 & 2 & 3 & 4 \\
\hline 0 & 1 & 2 & 3 & 4 \\
\hline 0 & 1 & 2 & 3 & 4
\end{tabular}

\begin{tabular}{|c|c|c|c|}
\hline 0 & 1 & 2 & 3 \\
\hline 0 & 1 & 2 & 3 \\
\hline 0 & 1 & 2 & 3 \\
\hline 0 & 1 & 2 & 3 \\
\hline
\end{tabular}

$\begin{array}{llll}0 & 1 & 2 & 3\end{array}$


19. I like the way I look

20. I'm not as smart as most people

21. I have difficulty concentrating

22. I'm satisfied with my sexual relationships

23.I am happy with my family relationships

24. I don't treat other people well

25. I am comfortable being around people

26. I can take care of myself in most situations

\begin{tabular}{llllr}
\hline 0 & 1 & 2 & 3 & 4 \\
\hline 0 & 1 & 2 & 3 & 4 \\
\hline 0 & 1 & 2 & 3 & 4 \\
\hline 0 & 1 & 2 & 3 & 4 \\
\hline 0 & 1 & 2 & 3 & 4 \\
\hline 0 & 1 & 2 & 3 & 4 \\
\hline 0 & 1 & 2 & 3 & 4 \\
\hline 0 & 1 & 2 & 3 & 4
\end{tabular}

During the past week how often did you ?

\begin{tabular}{|l|l|l|l|}
\hline & 5-7 days & 1-4 days & not at all \\
\hline $\begin{array}{l}\text { 1. Do your usual work (either inside or outside the } \\
\text { home ) }\end{array}$ & & & \\
\hline $\begin{array}{l}\text { 2. Get your work done as carefully and accurately as } \\
\text { usual. }\end{array}$ & & & \\
\hline $\begin{array}{l}\text { 3. take part in social, religious or recreation activities } \\
\text { (club meetings, movies, dancing, sports, parties, church) }\end{array}$ & & & \\
\hline $\begin{array}{l}\text { 4. Socialize with other people } \\
\text { 5. Care for yourself (bathe, dress, feed yourself) }\end{array}$ & & & \\
\hline & & & \\
\hline
\end{tabular}

\section{SECTION 5}

The following consists of a number of statements concerning how people deal with problems or difficulties. Please indicate with an $\mathbf{X}$ how often you use these approaches to deal with the problems you are presently experiencing.

\begin{tabular}{|c|c|c|c|c|c|}
\hline & never & very seldom & often & usually & always \\
\hline 1. Waiting to see what will happen & & & & & \\
\hline 2. Just taking one step at a time & & & & & \\
\hline 3. Standing your ground & & & & & \\
\hline $\begin{array}{l}\text { 4. Talking to someone who can do something concrete about } \\
\text { the problem }\end{array}$ & & & & & \\
\hline 5. Blaming yourself & & & & & \\
\hline 6. Feeling you change or grow as a person in a good way. & & & & & \\
\hline 7. Criticising or lecturing yourseif & & & & & \\
\hline 8. Avoiding being with people in general & & & & & \\
\hline 9. Asking someone you respect for advice and following it & & & & & \\
\hline $\begin{array}{l}\text { 10. Getting away from it for awhile, trying to rest or take a } \\
\text { vacation. }\end{array}$ & & & & & \\
\hline 11. getting the person responsible to change his or her mind. & & & & & \\
\hline
\end{tabular}


12. Telling yourself things that make you feel better.

13. Wishing you were a stronger person, more optimistic and forceful.

14. Concentrating on something good that can come out of the whole thing.

15. Maintaining your pride and keeping a stiff upper lip

16. Making light of the situation, refusing to get too serious about it.

17. Accepting understanding and sympathy from someone.

18. Coming up with a couple of solutions to the problem.

19. Rediscovering what is important in life.

20. Feeling bad that you cannot avoid the problem

21. Wishing that you could change the way that you feel.

22. Talking to someone to find out more about the situation.

23. Hoping a miracle will happen.

24. Wishing that you could change what has happened.

25. Thinking about fantastic or unreal things that make you feel better.

26. Bargaining or compromising to get something positive from the situation.

27. Changing something so things will turn out alright.

28. Feeling that time will make a difference, the only thing to

do is wait.

29. Feeling that you came out of the experience better than when you went in.

30. Accepting your strong feelings but trying not to let them interfere with other things too much.

31. Trying to make up for some of the bad things that have happened.

32. Feeling bad that you cannot avoid the problem.

33. Trying to make yourself feel better by eating, drinking, smoking or taking medication, etc.

34. Realising that you bring the problem on yourself.

35. Letting your feelings out somehow.

36. Doing something totally new that you never would have if this had not happened.

37. Looking for the silver lining, looking at the bright side of things.

38. Just concentrating on what you have to do next - the next step.

39. Keeping others from knowing how bad things are.

40. Going over the problem again and again in your mind to try and understand it.

41. Feeling you find new faith or important truth in life.

42. Taking a big chance or doing something really risky.

43. daydreaming or imagining a better time.

44. Getting angry at the people or things that caused the problem.

45. Turning to work or substitute activity to take you mind of things.

46. Accepting the next best thing to things that you wanted.

\begin{tabular}{|c|c|c|c|c|}
\hline never & $\begin{array}{c}\text { very } \\
\text { seldom }\end{array}$ & often & usually & always \\
\hline & & & & \\
\hline & & & & \\
\hline & & & & \\
\hline & & & & \\
\hline & & & & \\
\hline & & & & \\
\hline & & & & \\
\hline & & & & \\
\hline & & & & \\
\hline & & & & \\
\hline & & & & \\
\hline & & & & \\
\hline & & & & \\
\hline & & & & \\
\hline & & & & \\
\hline & & & & \\
\hline & & & & \\
\hline & & & & \\
\hline & & & & \\
\hline & & & & \\
\hline & & & & \\
\hline & & & & \\
\hline & & & & \\
\hline & & & & \\
\hline & & & & \\
\hline & & & & \\
\hline & & & & \\
\hline & & & & \\
\hline & & & & \\
\hline & & & & \\
\hline & & & & \\
\hline & & & & \\
\hline & & & & \\
\hline & & & & \\
\hline
\end{tabular}




\begin{tabular}{|l|l|l|l|l|l|}
\hline & never & very seldom & often & usually & always \\
\hline 47. Being inspired to do something creative. & & & & & \\
\hline 48. Talking to someone about how you are feeling. & & & & & \\
\hline 49. Sleeping more than usual. & & & & & \\
\hline $\begin{array}{l}\text { 50. Knowing what has to be done; doubling your efforts and } \\
\text { trying harder to make things work. }\end{array}$ & & & & & \\
\hline 51. Taking it out on other people. & & & & & \\
\hline 52. Getting professional help and doing what they recommend. & & & & & \\
\hline 53. Drawing on your past experiences. & & & & & \\
\hline 54. Making a plan of action and following it. & & & & \\
\hline $\begin{array}{l}\text { 55. Refusing to believe what had happened. Keeping your } \\
\text { feelings to yourself. }\end{array}$ & & & & & \\
\hline 56. Joking out it. & & & & & \\
\hline 57. Having fantasies or wishes about how things might turn & & & & & \\
\hline 58. Trying to forget the whole thing. & & & & & \\
\hline 59. Keeping your feelings to yourself. & & & & & \\
\hline 60. Not letting it get to you, refusing to think too much about it. & & & & & \\
\hline & & & & & \\
\hline
\end{tabular}

THANK YOU FOR PARTICIPATING IN THIS RESEARCH

Prishika Pillay 


\section{Appendix 3 - Questionnaires in Zulu}

Section 1: Brief Symptom Inventory

Section 2: Impact of Events Scale

Section 3: Recent Life Change Questionnaire

Section 4: Duke-UNC Health Profile

Section 5: Ways of Coping Checklist 
IMIBUZO EYIMFIHLO

Ungeyiphendule imibuzo ethize uma ufisa kanjalo.

Idethi

Igama lakho

Isibongo sakho

Ikheli lasekhaya

Ikheli yebhokisi

Inombolo wasengcino ekhaya

Inombolo wasengcino emsebenzeni

Ulimi owokuqala

Ubulili
iNgisis

iBhunu

uBesilisa
Isizulu

iXhosa

uBesifazane

Usuku lokuzalwa

Emingaka?

Ubuzwe:

$$
\begin{aligned}
& \text { oNsundu } \\
& \text { oMhlophe }
\end{aligned}
$$

iNdiya

Nomunye

iKhaladi

INkolo:

$\begin{array}{lc}\text { uMkrestu___ } & \text { iSulumani } \\ \text { uMjuda_ Nomunye }\end{array}$

uMhindu

Isimo Somshado:

Ongashadile

Ohlukanislie

Oshadile

uMfelwa

Umsebenzi owenzayo:

Sebenza ngokugewele

Sebenza ngokungagewele

Ngasebenzi

Thatha umhlalaphasi

Iliphi izinga lemfundo owaliphaha: Ngaphansi kwesikote esikhulu Qedite ibanga lokugana lesikole esikhulu iTechnical, noma isikole somsebenzi

Wadiwengulwa nini? 


\section{ISIQEPHU 1}

IKUKHATHAZE KANGAKANANI KULEZIZINSUKU EZIWU 7 EZEDLULE KANYE NOSUKU

LWANAMUBLA. Phendula yonke imibuzo. Kokelezela inombolo yaleyo naleyo nkinga ungeqi nokukodwa. Uma kwenzeka ushintsha mqondo yesula lokho osukukolezele kuqala. Funda isibonelo esenziwe ngaphansi / esilandelayo ngaphambi kokuthi uqhubeke.

$$
\begin{aligned}
& 0=\text { cha akwenzekanga } \\
& 1=\text { kancane }- \text { nje } \\
& 2=\text { phakathi naphakathi } \\
& 3=\text { kakhudlwana } \\
& 4=\text { kakhulu }- \text { kakhulu }
\end{aligned}
$$

\section{ISIBONELO}

Wawukhathazeke kangakanani ngalokhu:

Ukuza ubuhlunga emzimbeni.

\begin{tabular}{|l|l|}
\hline & \\
\hline WAWUKHATHAZEKE KANGAKANANI NGALOKHU: & \\
\hline 1. Ukuvevezela komzimba & 0 \\
\hline 2. Inzululwane & 0 \\
\hline 3. Umqondo wokuthi omunye umuntu angaphatha imicabango & 0 \\
\hline $\begin{array}{l}\text { 4. Uzizwe sengathi kungasolwa abantu ezinhluphekweni zakho } \\
\text { eziningi. }\end{array}$ & 0
\end{tabular}

5. Ukukhathazeka ngokukhumbula izinto.

6. Uzizwe ucanuka kalula noma uthunukwa wubala.

7. Ubuhlungu obusenhliziyweni noma obusesifubeni.

8. Uzizwe wesaba izindawo ezivulekile.

9. Imicabanga yokuqeda impilo yakho.

10. Uzwe sengathi bonke abantu abathembeile-neze.

11. Uugakthandi ukudla.

12. Uvele wesabe ngaphandle kwesizathu.

13. Ukuba nehliziyo encane ungakwazi ukuzibamba

14. Uzizwe unesizungu ngisho noma unanbantu.

15. Uzizwe uvimbekile ukuba ukwazi ukwenza izinto.

16. Uzizwe unesizungu.

17. Uzwe unomzimba ophansi.

18. Uzwe ungathandi lutho.

19. Uzizwe unokwesaba

20. Imizwa yakho ithunukala kalula.

21. Uzizwe sengathi abantu abezwani nawe noma abakuthandi.

22. Uzizwe ungaphansi kwabanye.

23. Uzwe kucanuzela inhliziyo.

24. Uzizwe sengathi ugadiwe noma abanye bakhuluma ngawe.

25. Uzwe ungakwazi ukulala.

26. Uzwe kufanele ubhekisise, uphinde futhi ubhekisise loko okwenzayo.

27. Ubunzima ekwenzeni izinqumo.

28. Uzizwe wesaba ukuhamba ngamabhasi noma ngezitimela.

\begin{tabular}{|c|c|c|c|c|}
\hline 0 & 1 & 2 & 3 & 4 \\
\hline & & & & \\
\hline 0 & 1 & 2 & 3 & $d$ \\
\hline 0 & 1 & 2 & $\frac{3}{3}$ & 4 \\
\hline 0 & 1 & 2 & 3 & 4 \\
\hline 0 & 1 & 2 & 3 & 4 \\
\hline 0 & 1 & 2 & 3 & 4 \\
\hline 0 & 1 & 2 & 3 & 4 \\
\hline 0 & 1 & 2 & 3 & 4 \\
\hline 0 & 1 & 2 & 3 & 4 \\
\hline 0 & 1 & 2 & 3 & 4 \\
\hline 0 & 1 & 2 & 3 & 4 \\
\hline 0 & 1 & 2 & 3 & 4 \\
\hline 0 & 1 & 2 & 3 & 4 \\
\hline 0 & 1 & 2 & 3 & 4 \\
\hline 0 & $I$ & 2 & 3 & 4 \\
\hline 0 & 1 & 2 & 3 & 4 \\
\hline 0 & 1 & 2 & 3 & 4 \\
\hline 0 & 1 & 2 & 3 & 4 \\
\hline 0 & 1 & 2 & 3 & 4 \\
\hline 0 & 1 & 2 & 3 & 4 \\
\hline 0 & 1 & 2 & 3 & 4 \\
\hline 0 & 1 & 2 & 3 & 4 \\
\hline 0 & 1 & 2 & 3 & 4 \\
\hline 0 & 1 & 2 & 3 & 4 \\
\hline 0 & 1 & 2 & 3 & 4 \\
\hline 0 & 1 & 2 & 3 & 4 \\
\hline 0 & 1 & 2 & 3 & 4 \\
\hline 0 & 1 & 2 & 3 & 4 \\
\hline 0 & 1 & 2 & 3 & 4 \\
\hline 0 & 1 & 2 & 3 & 4 \\
\hline 0 & 1 & 2 & 3 & 4 \\
\hline 0 & 1 & 2 & 3 & 4 \\
\hline & & & & \\
\hline
\end{tabular}

29. Ubunzima ekuphefumuleni.

30. Isivuru sokushisa noma ukubanda.

31. Uzwe kufanele ubalekele izinto ezithile, izindawo, nezimo ezithile ngoba zikwesabisa. 


$$
\begin{aligned}
& 0=\text { cha akwenzekanga } \\
& 1=\text { kancane }- \text { nje } \\
& 2=\text { phakathi naphakathi } \\
& 3=\text { kakhudlwana } \\
& 4=\text { kakhulu }- \text { kakhulu }
\end{aligned}
$$

\begin{tabular}{|l|l|l|l|l|l|}
\hline 32. Ingqondo yakho yehluleka ukuhumbula izinto. & 0 & 1 & 2 & 3 & 4 \\
\hline $\begin{array}{l}\text { 33. Ukuba - ndikindiki noma inkwashu ezingxenyeni ezithile } \\
\text { zomzimba wakho. }\end{array}$ & 0 & 1 & 2 & 3 & 4 \\
\hline 34. Umqondo wokuthi kufanele uhlawuliswe ngezono zakho. & 0 & 1 & 2 & 3 & 4 \\
\hline 35. Uzizwe ungento yalutho ngekusasa. & 0 & 1 & 2 & 3 & 4 \\
\hline 36. Unenkinga ekubekeni umqondo wakho entweni ethile. & 0 & 1 & 2 & 3 & 4 \\
\hline 37. Uzizwe ukhathele ezingxenyeni zomzimba wakho. & 0 & 1 & 2 & 3 & 4 \\
\hline 38. Uzizwe unovalo futhi unokwesaba & 0 & 1 & 2 & 3 & 4 \\
\hline 39. Imicabango yesifo nokufa. & 0 & 1 & 2 & 3 & 4 \\
\hline $\begin{array}{l}\text { 40. Ukuzwa kukhona okukupheplayo okuthi shaya, limaza noma } \\
\text { casula omunye umuntu. }\end{array}$ & 0 & 1 & 2 & 3 & 4 \\
\hline $\begin{array}{l}\text { 41. Kube khona okukuphehlayo okuthi yephula noma ubulale yonke } \\
\text { into. }\end{array}$ & 0 & 1 & 2 & 3 & 4 \\
\hline 42. Uzizwe uzenyeze uma unabanye. & 0 & 1 & 2 & 3 & 4 \\
\hline 43. Ukuzizwa ungakhululekile eningini. & 0 & 1 & 2 & 3 & 4 \\
\hline 44. Ungazizwa nakancane usondelene nabanye. & 0 & 1 & 2 & 3 & 4 \\
\hline 45. Uneziqubu zovalo nokwethuka. & 0 & 1 & 2 & 3 & 4 \\
\hline 46. Ukungena empikiswaneni njalo. & 0 & 1 & 2 & 3 & 4 \\
\hline 47. Ukuzizwa unokwesaba uma usele wedwa. & 0 & 1 & 2 & 3 & 4 \\
\hline $\begin{array}{l}\text { 48. Abanye bengakuniki ithuba lokukubonga ngokufanele kuloko } \\
\text { okwenze ngempumelelo. }\end{array}$ & 0 & 1 & 2 & 3 & 4 \\
\hline 49. Uzizwe ungenaso isikhundla, ungakwazi ukuhlala ukhululekile. & 0 & 1 & 2 & 3 & 4 \\
\hline 50. Ukuzizwa uyinto engelutho noma engenamsebenzi. & 0 & 1 & 2 & 3 & 4 \\
\hline 51. Uzizwe sengathi abantu bazokudelela uma ubathambela. & 0 & 1 & 2 & 3 & 4 \\
\hline 52. Ukuzizwa ulahlwe yicala. & 0 & 1 & 2 & 3 & 4 \\
\hline $\begin{array}{l}\text { 53. Ukuba nomqondo wokuthi kukhona okungalungile emqondweni } \\
\text { wakho. }\end{array}$ & 0 & 1 & 2 & 3 & 4 \\
\hline & & & & & \\
\hline
\end{tabular}

\section{ISIQEPHU 2}

Ngo.. .wehlelwa. ..ISILINGANISO
(Usuku)
1.Cha akwenzekanga
(Isigameko)
2.Akuvamisile
3.Kwesinye isikhathi 4.Kuvamisile

Okubhalwe ngaphansi uhla lokushiwo abantu emuva kokuhlangabezana nezimo ezinzima ezimpilweni zabo. Uyacelwa-ke ukuthi ufunde ngakunye bese uyakhombisa ukuthi loku abakushoyo kuvamise kangakanani kuwe EZINSUKWINI EZIYISIKHOMBISA EZEDLULE. Uma kungenzekanga ngaleso sikhathi, uyacelwa ukuthi ubeke isiphambano ku-"cha akwenzekanga"

\begin{tabular}{|l|c|c|c|c|}
\hline 1. Ngacabanga ngako ngesikhathi ngingakuqondile & 1 & 2 & 3 & 4 \\
\hline $\begin{array}{l}\text { 2. Ngakuvikela ukuthi ngithukuthele/ngidinwe ngesikhathi } \\
\text { ngicabanga ngako noma ngesikhathi ngikukhumbula }\end{array}$ & 1 & 2 & 3 & 4 \\
\hline 3. Ngazama ukukususa emqondweni & 1 & 2 & 3 & 4 \\
\hline
\end{tabular}


1. Cha akwenzekanga

2. Akuvamisile

3. Kwesinye isikhathi

4. Kuvamisile

\begin{tabular}{|l|c|c|c|c|}
\hline $\begin{array}{l}\text { 4. Ngaba nenkinga yokuphathwa ubuthongo noma yokugcineka } \\
\text { ngilele ngoba imifanekiso noma imicabango ngako yayiloku ifika } \\
\text { enggondweni yami }\end{array}$ & 1 & 2 & 3 & 4 \\
\hline 5. Nginezikhawi ezinzima zemizwa yako & 1 & 2 & 3 & 4 \\
\hline 6. Ngaba namaphupho ngako & 1 & 2 & 3 & 4 \\
\hline 7. Ngaziqhelelanisa nokwakungangikhumbuza ngako & 1 & 2 & 3 & 4 \\
\hline 8. Ngezwa sengathi akwenzekanga noma & 1 & 2 & 3 & 4 \\
\hline 9. Ngazama ukukhuluma ngako & 1 & 2 & 3 & 4 \\
\hline 10.Imifanekiso ngako yavela emqondweni wami & 1 & 2 & 3 & 4 \\
\hline 11.Ezinye izinto zangenza ngaqhubeka ukucabanga ngako & 1 & 2 & 3 & 4 \\
\hline $\begin{array}{l}\text { 12.Ngacacelwa ukuthi ngangiloku nginayo imizwa ngako kodwa } \\
\text { angizange ngenze lutho ngayo }\end{array}$ & 1 & 2 & 3 & 4 \\
\hline 13.Ngazama ukungacabangi ngako & 1 & 2 & 3 & 4 \\
\hline 14.Noma yikuphi okuyisikhumbuzo kwayibuyisa imizwa ngako & 1 & 2 & 3 & 4 \\
\hline $\begin{array}{l}\text { 15.Imizwa yami ngako yayingathi indikindiki Ngiyabonga } \\
\text { ngokuphendula kwakho yonke lemibuzo }\end{array}$ & 1 & 2 & 3 & 4 \\
\hline & & & & \\
\hline
\end{tabular}

\section{ISIQEPHU 3}

Ukuphendula imibuze engezansi, beka uphawu esikweleni esisodwa noma ngaphezulu esandleni sokudla kulowo nalowo mbuzo. Uma isigameko esibuziwe senzeka eminyakeni emibili eyedlula, khombisa ukuthi senzeka nini ngokubeka uphawu esikhaleni esifanele: ezinyangeni ezi 0-6, 7-12 ezedlule, njalonjalo. Uma isigameka sakwehlela ngaphezulu kokukodwa eminyakeni emibili eyedlule, beka uphawu kuzo zonke izikwele ezifanele, Uma isigameko singazange sikwehlele eminyakeni emihlanu eyedlule (noma singakaze sikwehlele), shiya zonke izikwele zingenalutho. KULAMABINZA EZIKHATHI EZIBEKWE, WAKE WABA:

\begin{tabular}{|l|l|l|l|l|}
\hline Izinyanga ezedlule & $19-24$ & $13-18$ & $7-12$ & $0-6$ \\
\hline EZEMPILO & & & & \\
\hline Isifo noma ukulimala okwa: & & & & \\
\hline $\begin{array}{l}\text { kugcina usembedeni isonto noma ngaphezulu nama noma ngaphezulu } \\
\text { nama kwadingeke usiwe esibhedlela? }\end{array}$ & & & & \\
\hline Sasingenzima kunesibhalwe ngenhla? & & & & \\
\hline Umsebenzi okhulu ophathelene namazinyo? & & & & \\
\hline Ushintsho olukhulu oluphathelene nemikhutshana yokudla? & & & & \\
\hline Ushintsho olukhulu oluphathelene nemikhutshana yokulala? & & & & \\
\hline $\begin{array}{l}\text { Ushintsho olukhulu ohlotsheni futhi/ noma nasezingeni } \\
\text { lokuzijabulisa? }\end{array}$ & & & & \\
\hline & & & & \\
\hline
\end{tabular}




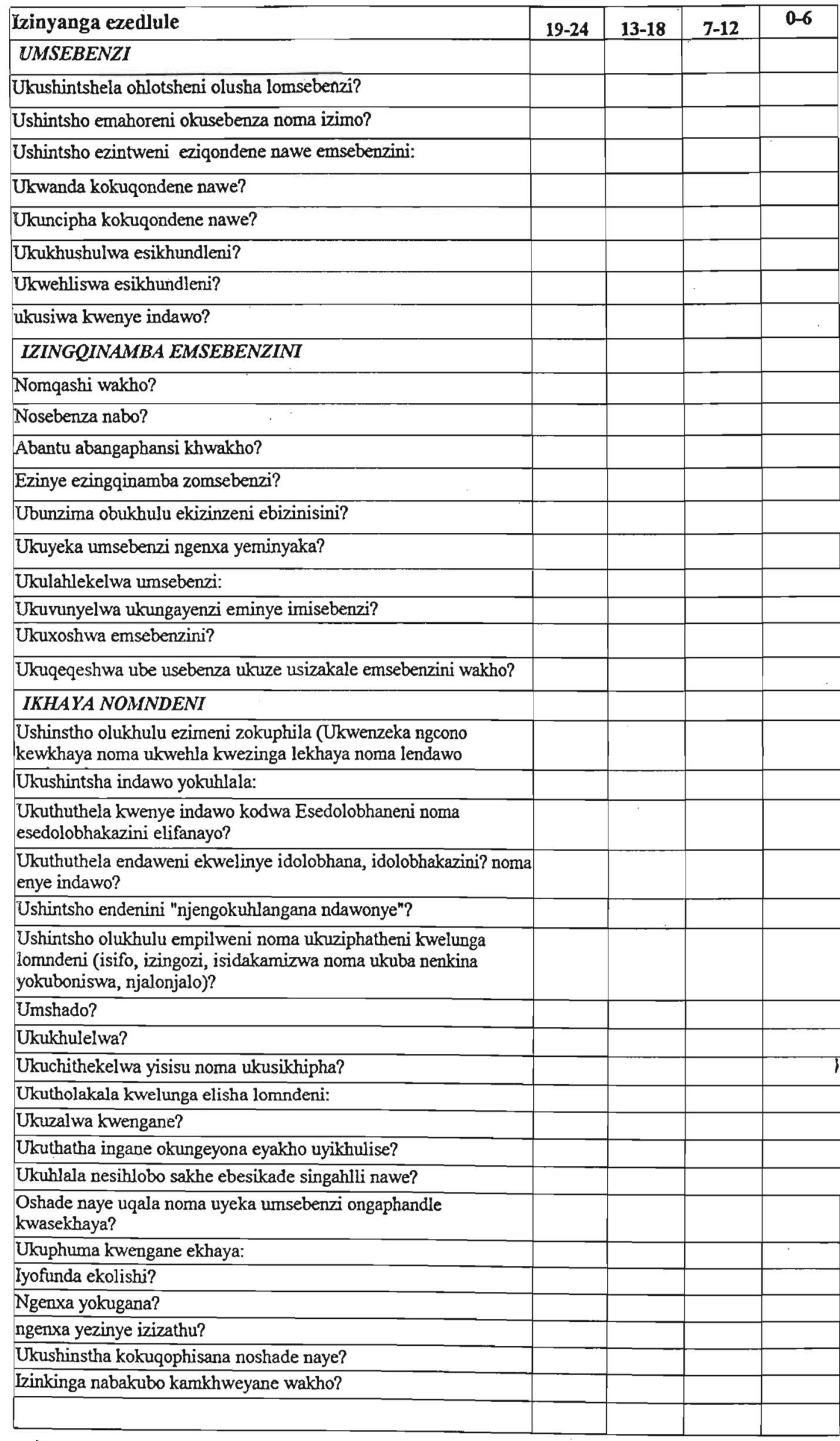




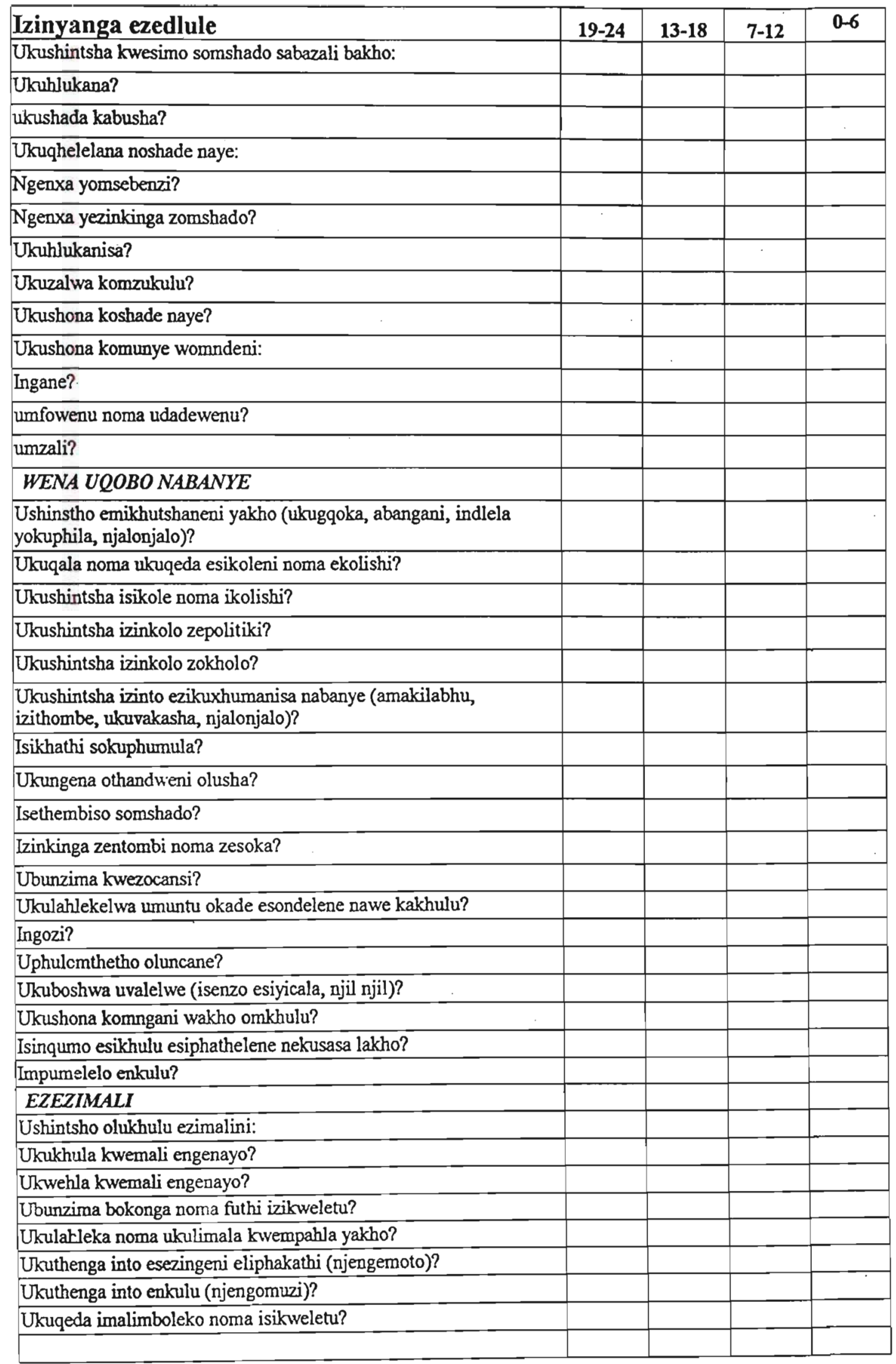




\section{ISIOEPHU 4}

Lapha kunemibuzo ephathelene nempilo kanye nemizwa yakho. Uyacelwa ukuba ufunde umbuzo ngamunye ngokucophelela bese unukeza impendulo yakho ezedlula zonke. Azikho izimpendulo ezifanele nezingafanele.

Esontweni eledlule:

Jbe nenkinga engakanani ngaloks:

\begin{tabular}{|l|l|l|l|}
\hline & Lutho & Nje & Kakhulu \\
\hline 1. Ukubona & & & \\
\hline 2. ukuzwa & & & \\
\hline 3. Ukukhuluma & & & \\
\hline 4. Ukuzwa amaphunga amabi & & & \\
\hline 5. Ukuzwa ukudla & & & \\
\hline 6. Uthando lokudla & & & \\
\hline 7. Ukuhlafuna & & & \\
\hline 8. Ukugunya & & & \\
\hline 9. Ukuzikhulula & & & \\
\hline 10. Ukuchama & & & \\
\hline 11. Ukuphefumula & & & \\
\hline 12. Ukulala & & & \\
\hline 13. Ukuhamba & & & \\
\hline 14. Ubuhlungu bekhanda & & \\
\hline 15. Ubuhlungu noma ukunkenkethe kunoma yiyiphi & & & \\
\hline ingxenye yomzimba & & & \\
\hline 16. Ukunkenketha kunoma yiyiphi ingxenye & & & \\
\hline yomzimba wakho & & & \\
\hline 17. Ukungagayeki kokudla & & & \\
\hline 18. UmkhuhlanelImfiva & & & \\
\hline 19. Ukukhathala kalula & & & \\
\hline 20. Ukuquleka & & & \\
\hline 21. Ukukhoblwa & & & \\
\hline 22. Ubuntekenteke kunoma yiziphi izingxeny & & & \\
\hline 2omzimba & & \\
\hline 23. Ukuzizwa ukhathazekile/ ubhulungu emoyeni & & & \\
\hline 24. Ukwesaba okkukhulu/uvalo & & & \\
\hline
\end{tabular}

Enyangeni eyedlule:

Ube nenkinga engakanani ngaloku:

\begin{tabular}{|l|l|l|l|}
\hline & Lutho & Nje & Kakhulu \\
\hline 25. Ukwehla emzimbeni & & & \\
\hline 26. Ukukhuluphala/ ukuzimuka & & & \\
\hline 27. Ukopha okungavamisile & & & \\
\hline 28. Umdlandla kwezocansi & & & \\
\hline & & & \\
\hline
\end{tabular}


Esontweni eledlule:

1. Izinsuku ezingaki usembedeni/ ulele ingxenye enkulu yosuku ngenxa yesifo, ukulimala noma inkinga yempilo?

\begin{tabular}{|l|l|l|}
\hline Lutho & $1-4$ izinsuku & $5-7$ izinsuku \\
\hline
\end{tabular}

2. Izinsuku ezingaki uhleli ekhaya lakho ngenxa yesifo,ukulimala noma inkinga yempilo?

\begin{tabular}{|l|l|l|}
\hline Lutho & 1-4 izinsuku & 5-7 izinsuku \\
\hline
\end{tabular}

3. Ube nobunzima obungakanani ekuhambeni uzungeze ikhaya lakho ngenxa yesifo, ukulimala noma inkinga yempilo?

\begin{tabular}{|l|l|l|l|}
\hline Lutho Nje & Kakhulu & Angkwasi & ukuphumela phandle \\
\hline
\end{tabular}

Namhlanje ingabe unayo inkinga noma ubunzima emzimbeni:

\begin{tabular}{|l|l|l|l|}
\hline & Lutho & Nje & Kakhulu \\
\hline 4. Ukugijima ama-mayela amahlanu Lutho Nje Kakhulu & & & \\
\hline +5. Ukubamba umntwana & & & \\
\hline 6. Ukuhamba ezitebhisini ezihambayo & & & \\
\hline 7. Ukuzwa umsakazo noma umabonakude & & & \\
\hline 8. Ukugijima imayela elilodwa & & & \\
\hline +9.Ukuhamba ibanga elilinganiselwa enkundleni yebhola & & & \\
\hline (amayadi ayi-100) & & & \\
\hline 10. Ukuya endlini yokugezela & & & \\
\hline 11. Ukucwcwa i-apula & & & \\
\hline +12.Ukufund uphawu lomgwaqo & & & \\
\hline 13. Ukugijima ibanga elilinganiselwa enkundleni yebhola & & & \\
\hline (amayadi ayi-100) & & & \\
\hline 14. Ukufunda iphephandaba & & & \\
\hline 15. Ukukama izinwele zakho & & & \\
\hline & & & \\
\hline
\end{tabular}

Lapha kunezitatimende ongazisebenzisa ukuchaza ukuthi uzizwa unjani. Uyacelwa ukuba ufunde isitatimende ngasinye ngokucophelela bese ubeka isiphambano esikhaleni esichaza kangcono okushiwo yisitatimende ngawe.

Nasi-ke isibonelo:

\begin{tabular}{|c|c|c|c|}
\hline & $\begin{array}{l}\text { Singichaza } \\
\text { ngqo }\end{array}$ & $\begin{array}{c}\text { Siyangicha-za } \\
\text { chaza nge }\end{array}$ & $\begin{array}{l}\text { Asingichazd } \\
\text { neze }\end{array}$ \\
\hline $\begin{array}{l}\text { Ngiyawuthanda umculo wezinsimbi oboniwsa } \\
\text { kumabonakude }\end{array}$ & & & \\
\hline
\end{tabular}


Phendula konke okulandelayo ngawo wonke amandla akho. Khumbula ayikho impendulo efanele nengafanele.

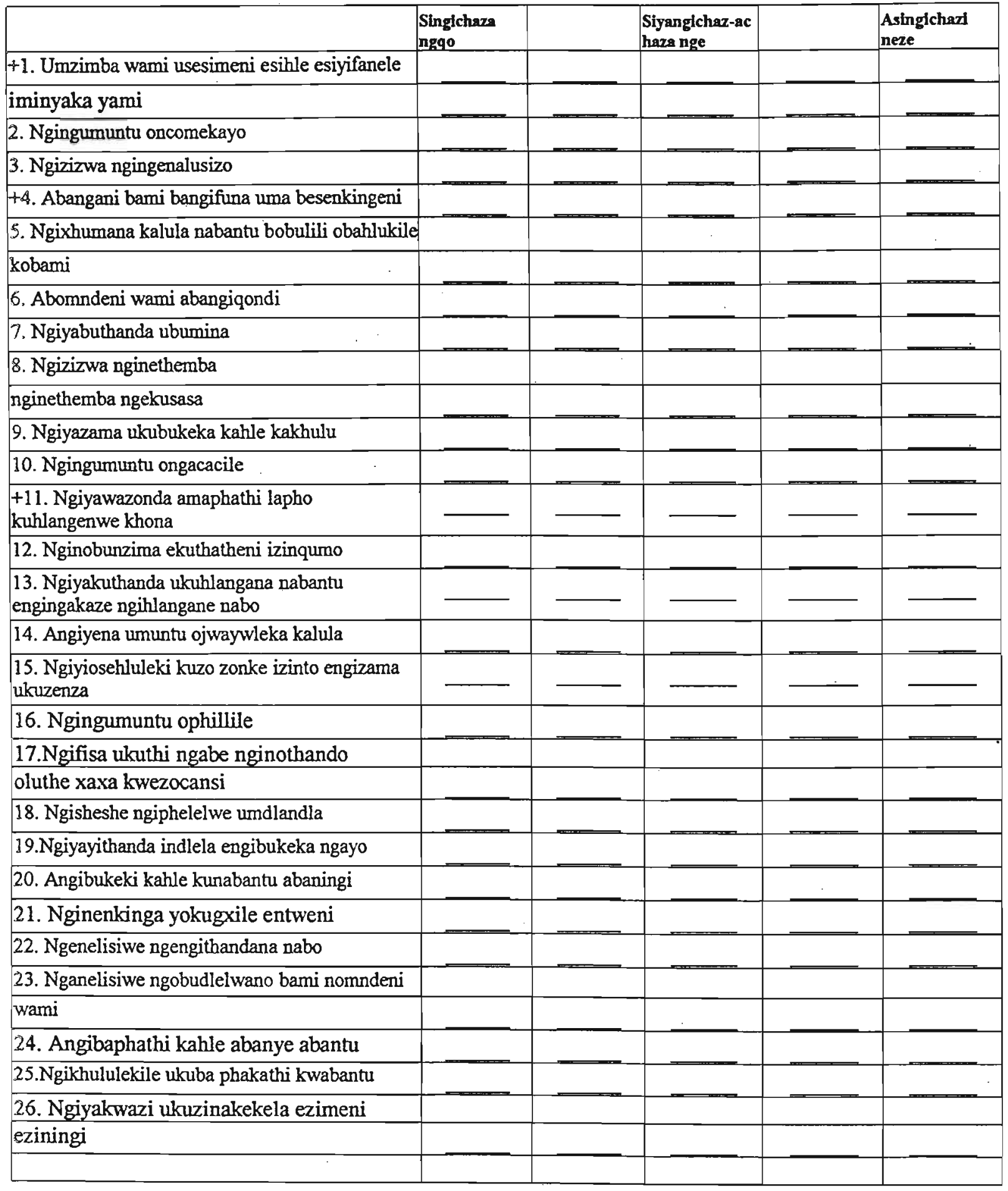


Evikini eledlule kukangakanani:

1. Uvamisile ukusebenza(ngaphakathi noma ngaphandle kwasekhaya)

\begin{tabular}{|l|c|c|c|}
\hline Lzinsuku ezingu: & $5-7$ & $1-4$ & Neze \\
\hline
\end{tabular}

2. Uqueda umsebenzi ngokucophelela kahle ngokujwayelekile

\begin{tabular}{|l|c|c|c|}
\hline Izinsuku ezingu: & $5-7$ & $1-4$ & Neze \\
\hline
\end{tabular}

3. Ubamba iqhaza lakublangenwe khona, enkolweni, noma ezintweni zokuzijabulisa (emihlanganweni yamakilabhu, ezithombeni, emidansweni, emidlalweni emaphathini, esontweni)

\begin{tabular}{|l|c|c|c|}
\hline Izinsuku ezingu: & $5-7$ & $1-4$ & Neze \\
\hline
\end{tabular}

4.Ukuzimbanakanya nabanye abantu

\begin{tabular}{|c|c|c|c|}
\hline Izinsuku ezingu: & $5-7$ & $1-4$ & Neze \\
\hline \multicolumn{4}{|c|}{ 5. Ukuzinakekela (ukuzigeza, ukuzigqokisa, nokuzidlela) } \\
\hline Izinsuku ezingu: & $5-7$ & $1-4$ & Neze \\
\hline
\end{tabular}

\section{ISIOEPHU 5}

Lamakhasi alandelayo anezitatimende eziqondene nendlela ethile abantu ababgaphatha ngayo izinkinga zabo noma ubunzima. Uyacelwa ukuba ukhombe kulamaphoyinti ukuthi wejwayele kangakanani uku sebenzisa lezizindlela ezilandelayo zokubhekana neizinkinga onazo njengamanje.

\begin{tabular}{|c|c|c|c|c|c|}
\hline $\begin{array}{l}0 \text { - angikase } \\
1 \text { - angivamisile } \\
2 \text { - ngivamisile }\end{array}$ & & & & & \\
\hline 1. Ngilindele ukubona ukuthi kuzokwenzekani. & 0 & 1 & 2 & 3 & 4 \\
\hline 2. Izinto ngizithatha ngayinye ngesikhathi. & 0 & 1 & 2 & 3 & 4 \\
\hline 3. Uyazabalaza ushaye phansi ngonyawo ulwele lokho okufunayo. & 0 & 1 & 2 & 3 & 4 \\
\hline $\begin{array}{l}\text { 4. Ukukhuluma nomunye umuntu ongakwazi ukwenza okuthile } \\
\text { okubonakalayo ngenkinga. }\end{array}$ & 0 & 1 & 2 & 3 & 4 \\
\hline 5. Uzilahle wena ngecala. & 0 & 1 & 2 & 3 & 4 \\
\hline 6. Uzizwe ukhulile ngengqondo. & 0 & 1 & 2 & 3 & 4 \\
\hline 7. ukuzikhuza noma ukuzifundisa wena. & 0 & 1 & 2 & 3 & 4 \\
\hline 8. Ukugwema ukuba nabantu. & 0 & 1 & 2 & 3 & 4 \\
\hline 9. Ukucela omhloniphayo ukuba akunike iseluleko bese usilandela. & 0 & 1 & 2 & 3 & 4 \\
\hline \multicolumn{6}{|l|}{ 10. Ukubalekela isimo esithile ngokuhlaba ikhefu noma ngo-kuphumula. } \\
\hline 11. Ukuthola umnutu okwasi ukuguqula umgondo wakhe. & 0 & 1 & 2 & 3 & 4 \\
\hline 12. Ukuzitshela izinto onokwazi ukuthi uzizwe ngcono. . & 0 & 1 & 2 & 3 & 4 \\
\hline $\begin{array}{l}\text { 13. Unesifiso sokuthi ngabe kungcono ukube wawungumuntu onamandla, } \\
\text { ulangazelele amathuba amahle kuphela empilweni, ubenamandla okuphoqa } \\
\text { izimo. }\end{array}$ & 0 & 1 & 2 & 3 & 4 \\
\hline 14. Ukubeka umqondo wakho ezintweni ezinhle ezingavela esimweni sonke. & 0 & 1 & 2 & 3 & 4 \\
\hline 15. Ugcine izinga lakho lokuziqhenya lisezingeni lalo, uzizwe umkhulu. & 0 & 1 & 2 & 3 & 4 \\
\hline 16. Ukuthetha noma yisiphi isimo kalula-nje. uvimbe ukuzindla ujule ngaso. & 0 & 1 & 2 & 3 & 4 \\
\hline 17. Ukwemukela ukuqqondo kwabanye-abantu futhi ube nozwela nomunye. & 0 & 1 & 2 & 3 & 4 \\
\hline 18. Ukuqhamuka namasu amaningana ehlukahlukene okuxazulula inkinga. & 0 & 1 & 2 & 3 & 4 \\
\hline 19. Ukuthola kabusha okubalulekile empilweni. & 0 & 1 & 2 & 3 & 4 \\
\hline 20. Uzizwe ungaphathekile kahle ngenxa yokwehluleka ukugwena inkinga. & 0 & 1 & 2 & 3 & 4 \\
\hline $\begin{array}{l}\text { 21. Unesifiso sokuthi kungabe kungcono ukuba uyakwasi ukuguqula isimo } \\
\text { ozizwa okusona ngaleso sikhathi }\end{array}$ & 0 & 1 & 2 & 3 & 4 \\
\hline 22. Ukuxoxisana nomunye umuntu ukuze uthole okuningi mayelana nesimo. & 0 & 1 & 2 & 3 & 4 \\
\hline
\end{tabular}


0 - angikase

1 - angivamisile
2 - ngivamisile

3 - ngijwayele

4 - njalo

23. Ubenethemba lokuthi kuzokwenzeka into eyisimangaliso-nje.

24. Ubenesifiso sokuthi kwakungaba kuhle ukube wawungaba namandla okuguqula osekwenzekile.

25. Uzizwe ucabanga ngezinto ezinhle eziyisimanga noma okungese kube yiqiniso ukuthi zenzeke kodwa ezingakwazi ukuthi zikwenze ngcono.

26. Uzikhandle noma uzikhathaze kangangokuba uze ulahlekelwe mgokuthize ukuze ulungise isimo.

27. Uguqule okuthize ukuze izinto zihambe kahle.

28. Uzwe-nje ukuthi mhlawumbe isikhathi singawenza umehluko, ngakho-ke ofanele ukukwenzakuphela ukulinda.

29. Uzizwe kwesinye isikhathi ukuthi ukuhlangabezana nesimo esithize esikwenze waba ngcono kunesikhathi lapho wawusaqala ukuhlangabezana malesimo.

30. Uzizwe wemukele imzwa yakho eqinile kodwa ungavumeli ukuba ithikameze kakhulu ezinye izinto.

31. Uma kwenzekile okungekuhle uzame ukukulungisa ukuze kukuyele esimweni esifanayo nesejwayelekile.

32. Uphatheke kabi ukuthi awukwazanga inkinga.

33. Ukuzenza wena uzizwe ngcono ngokudla, ukuphuza, ukubhema noma ukusebenzisa noma iyiphi inhlobo yomuthi.

34. Ubone ukuthi nguwe ozidalela inkinga.

35. Uma kwenzeka ukuthi ungaphatheki kahle wazise abanye ngalokho.

36. Uzithole wenza into entsha obungeke uyenze ukuba awuhlangananga nesimo obhekene naso.

37. Uzithole uphokophelele ezintweni ezingcono kuphela empilweni.

38.Ugxile kulokho usuke usuzokwenza.

39. Ukungazisi abanye indlela isimo esibi ngayo.

\begin{tabular}{|l|l|l|l|l|}
\hline 0 & 1 & 2 & 3 & 4 \\
\hline 0 & 1 & 2 & 3 & 4 \\
\hline
\end{tabular}

40. Ukininga uzindle ngenkinga njalo, uzama ukuyiqondo noma ukuyazi.

41. Uzizwe unenkolelo entsha noma uzizwe uthole iqiniso elisha empilweni yakho.

42. Uthatha ushani omkhulu noma wenza into eyingcuphe enkulu.

43.Uzibone uhhema-nje ngezinto ezingasoze zenzeka okukanye ubuke ngamehlo engqondo isikhathi esihle empilweni yakho.

44. Uzizwe kuthi sangana ngolaka kubantu noma ezintweni ezidala inkinga.

45. Wenza umsebenzi mumbe okukanye wenze okuthize ukuze wenze umgondo wakho ukhohlwe yizinkinga.

46. Uzithole wemukela into engaphansana-nje kodade uyidinga.

47. Uzizwe unomudia wokwenza okuthile okwakhayo.

48. Ukhulume nomunye umuntu ngendlela ozizwa uyiyona.

49. Ulala isikhathi eside kunesejwayelekile. \begin{tabular}{|l|l|l|l|l|l|}
\hline 49. Ulala isikhathi eside kunesejwayelekile. & 0 & 1 & 2 & 3 & 4 \\
\hline $\begin{array}{l}\text { 50. Wasi okufanele kwenzive, wenze imizamo yakho ngokuphindwe kabili, } \\
\text { uzama kanzima ukuze izinto zihambe kahle. }\end{array}$ & 0 & 1 & 2 & 3 & 4
\end{tabular}

51. Uma kunenkinga uyikhiphele kwabanye abantu.

52. Uthole usizo olunzulu wenze njengoba ochwepheshe bencoma.

53. Usebenzise ulwazi lwakho lwalokho ohlangabezene nakho esikhathine esedlule, ekwenzeni izinto manje.

54. Uzibone wenza icevo lezinto ofanele ukuzenza bese uyalilandla.

55. Uzithole wenqaba ukuthi kukhona okwenzekile ugcine imizwa yakho kuwena.

56. Uzithole wenza ihlaya ngokwenzekile.

57. Uzithole ucabanga ngezinto eziyisimanga noma unezifiso ezithize ozifisela ukuthi izinto ziguquke zibe yizona.

58. Uzame ukukhohlwa yinkinga.

59. Ukugcina imizwa yakho kuwena.

\begin{tabular}{|c|c|c|c|c|}
\hline 0 & 1 & 2 & 3 & 4 \\
\hline 0 & 1 & 2 & 3 & 4
\end{tabular}

\begin{tabular}{|c|c|c|c|c|}
\hline 0 & 1 & 2 & 3 & 4 \\
\hline 0 & 1 & 2 & 3 & 4 \\
\hline
\end{tabular}

\begin{tabular}{|l|l|l|l|l|}
0 & 1 & 2 & 3 & 4 \\
\hline 0 & 1 & 2 & 3 & 4
\end{tabular}

\begin{tabular}{|c|c|c|c|}
\hline 0 & 1 & 2 & 3 \\
\hline
\end{tabular}


Appendix 4 - Letter to Participants 


\section{Dear Participant}

The incidence of rape in our country is increasing drastically. There are many people like yourself who have to deal with both the short term and long term consequences. Rape, because of these consequences, is not only affecting individuals but our entire society.

Rape survivors are at risk of contracting various infectious diseases, which could have an adverse effect on ones health. This research would enable researchers to devise appropriate intervention strategies which would facilitate positive health outcomes, and thus benefit all rape survivors.

Participation in this research requires you to provide answers to a questionnaire and to donate a sample of your blood, which would be used to assess your physical health status. You would be required to fulfil this requirement on three different times:

1) within 5 days of the rape

2) 15 days after the rape

3) 35 days after the rape

The blood sample would be drawn at the district surgeons office in Ebenezer Street. It is important to note that drawing of blood samples might induce a level of pain and fear. However you would be contributing towards helping other rape survivors like yourself. If you do decide to participate in this research all information supplied by you would be treated confidentially and you would be free to decline or withdraw at any time without suffering and disadvantage or prejudice to treatment.

Your participation in this research is highly appreciated.

Yours sincerely

Prishika Pillay 


\title{
MHLANGANYELI OTHANDEKAYO
}

Izinga lokudiwengula kulelizwe lenguka nejesiznimi esesabisayo. Baningi abantu abafana nawe okunye kudingeke ukuba babhekane nemiphu mela yako ema kwesihathi esifushane noma eside kwenzekile. Ukundlwengula ngenxa yalemiphumela, akuhlukumezi umuntu oyedwa kudwa umphakathi wonkana.

Abadlwengulula obasindayo basengozini yokuhlangabezama nezifo ezinhlobonhlobo ezithathelanayo ezingaba nobubi empihweni yomuntu. Uewaningo lungaba wusizo ekutholeni izindbela zonkuvikela izifu, ngaleyondlela kusizakale bonke abasindile ekudlwengulweni.

Uewaningo ludinga uphendule imibuzo bese unikela ngesampula uphendule imibuzo bese unikela ngesampula yegazi lakho ezosetshemziselwa ukukola ubunjalo bempilo yakho. Kuyodingeka ukwenze lokhu ngezikhathi esintathu ezahlukene :

1. Izinsuku ezinhlanu (5) emva kokudlwengulwa.

2. Izinsuku eziyi shumi nonhlanu (15) emva kokudlewengulwa.

3. Izinsuku ezingamashumi amathathu nonlanu evma kokudlewengulwa.

Isampula legazi liyothathelwa endlini yoku cwaningela izifo. Qaphela ukuthi ukthathano kwamasampula egezi kungase kuhambisane nobulkingu kanye nokwesaba. Nokho kuyoba uphonsa itshe esivivaneni ekusizeni abadlwengulwa abasindayo abafana nawenje. Uma nquma ukubamba iqhaza ocwaningweni lonke uwazi olulethwe nguwe luygcinwa luyisifuba. Kuyoba kuwena futhi ukuba wenqabe nome uhoxe nome nini ngaphandle kokuba ukwelashwa kwakho kuhluku mezeke okanye kube nenguyazene ethize.

Iqhaza lakho ocwaningweni liyothokozelwa kakhulu.

Yimina okuthandayo

\author{
Prishika Pillay \\ tel: $\quad 0331-73181 / 76807(\mathrm{a} / \mathrm{h})$ \\ cell: $\quad 0835602686$
}


Appendix 5 - Consent Form 
Mina, (igama) ngiyavuma ukubamba iqhaza

kulolucwaningo olulandelayo.

Ngiyakuvuma ukuthi ngazisiwe:

U-Prishika Pillay

Ipoyisa

Udokotela

Omunye.

ngokuphathelene nokungaba ubuhle nobubi okungahle kubangwe yinqubo.

Mina, (igama) ngiyavuma ukuthi ngiyaqonda futhi ngiyavumelana nolwazi enginikezwe lona maqondana naloku okuzozanywa.

Ngiyavuma ukuthi lolucwaningo olungenhla luzoqhutshwa u-Prishika Pillay enganyelwe u-profesa G.C. Lindegger kanye no profesa L. Schlebush.

Ngiyazi ukuthi nginelungelo lokusihoxisa isinqumo sami noma ngasiphi isikhathi ngaphandle kokubeka ukunakekelwa kwami engcupheni.

Isayinwe: (ozobamba iqhaza). Usuku

Isayinwe: (ufakazi).

Usuku

Isayinwe: (ongamele).

Usuku

Isayini: (Umcwaningi).

Usuku 


\section{INFORMED CONSENT}

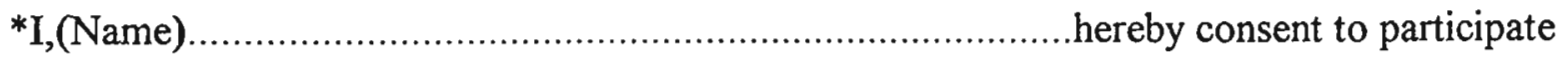
in the research procedure being conducted on myself or the person indicated in (iv) below:

* I acknowledge that I have been informed by:

(Name)...

concerning the possible advantages and possible adverse effects which may result from participating in this research.

*I,(Name).

hereby acknowledge that I understand and accept the Information given to me in connection with this research.

*I agree that the research will be cariied out by Ms Prishika Pillay and will be supervised by Professor G.C. Lindegger (University of Natal - Pmb) and Professor L. Schlebush (University of Natal - Medical School)

*I acknowledge that I understand the contents of this form, including the information provided in the "Information to Participants" leaflet and as the

\section{PARTICIPANT OTHER (specify)}

freely consent to (Name)................................................................... participating in the research.

*I am aware that I may withdraw my consent at any time without prejudice to further care.

Signed:

Date:

Participant / Other

Signed:

Date:

Witness

Signed:

Date:

Informant

Signed:

Pilla

Researcher

Date: 
Appendix 6 - Figure 8 : Participants Immune Parameters Across Time

$-155$ 


$\begin{array}{rrrrrr} & 1 & 2 & 3 & 4 & 5 \\ \text { T1 } & 66.8 & 80 & 75.8 & & 83.9 \\ \text { T 2 } & 58.8 & 81.4 & 75.8 & & 88.8 \\ \text { T 3 } & 61.5 & 79.8 & 81.4 & 76.6 & 89.9\end{array}$

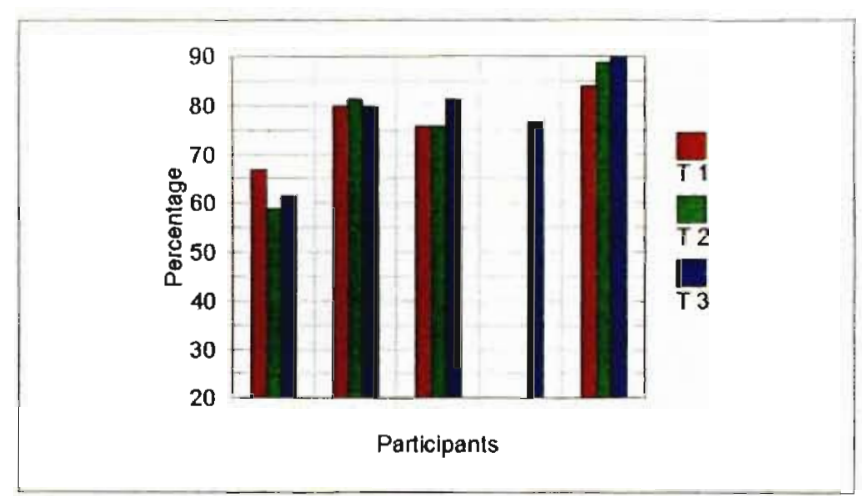

Figure $8 a$ : CD $3 \%$ Across Time

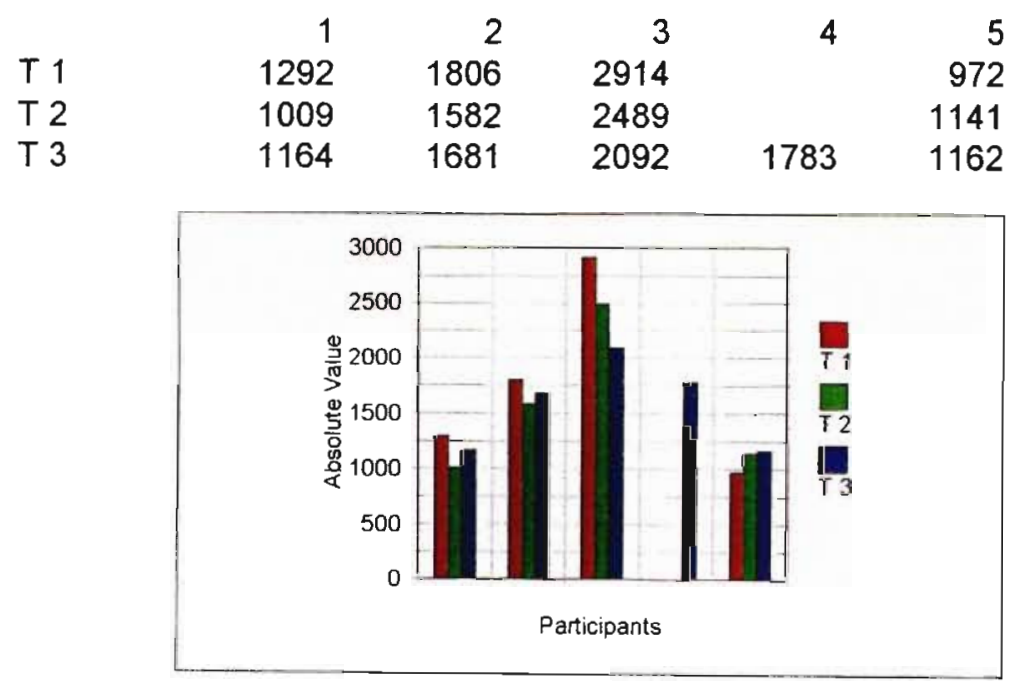

Figure 8b: CD 3 Absolute Across Time

$\begin{array}{rrrrrr} & 1 & 2 & 3 & 4 & 5 \\ \text { T 1 } & 39.1 & 51 & 50.2 & & 16.2 \\ \text { T 2 } & 37.7 & 46.6 & 43 & & 15.2 \\ \text { T 3 } & 42.4 & 48.8 & 52.3 & 29.3 & 20.4\end{array}$

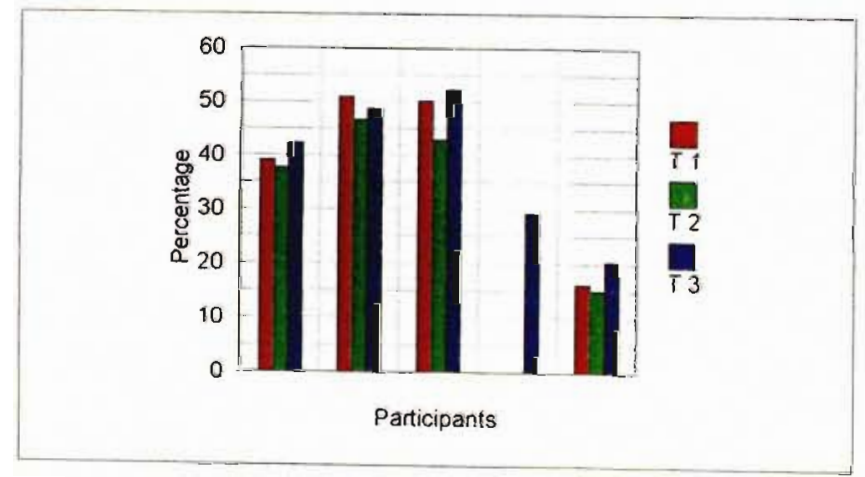

Figure 8c: CD $4 \%$ Across Time 


$\begin{array}{rrrrrr} & 1 & 2 & 3 & 4 & 5 \\ \text { T 1 } & 756 & 1151 & 1930 & & 188 \\ \text { T 2 } & 647 & 906 & 2017 & & 195 \\ \text { T 3 } & 802 & 1028 & 1304 & 682 & 266\end{array}$

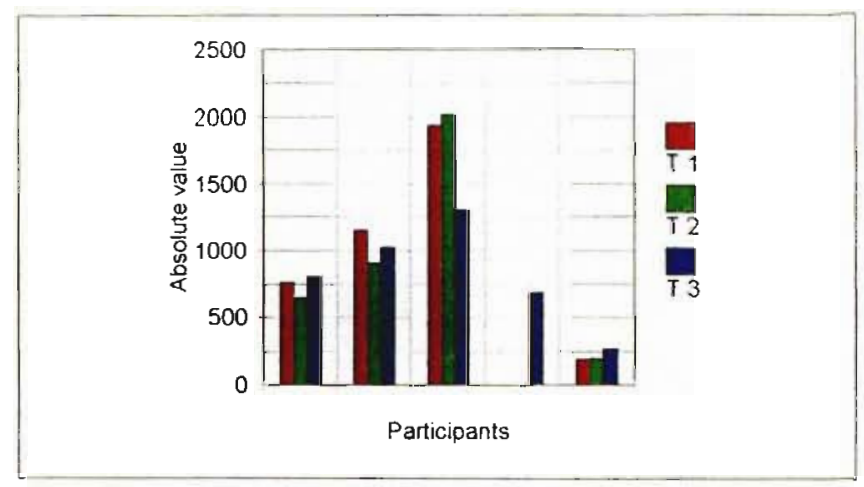

Figure 8d: CD 4 Absolute Across Time

$\begin{array}{rrrrrr} & 1 & 2 & 3 & 4 & 5 \\ \text { T 1 } & 18.3 & 30.3 & 28.8 & & 56.4 \\ \text { T 2 } & 14.5 & 36.5 & 32.2 & & 66.9 \\ \text { T 3 } & 16.1 & 30.6 & 32.6 & 48 & 66.8\end{array}$

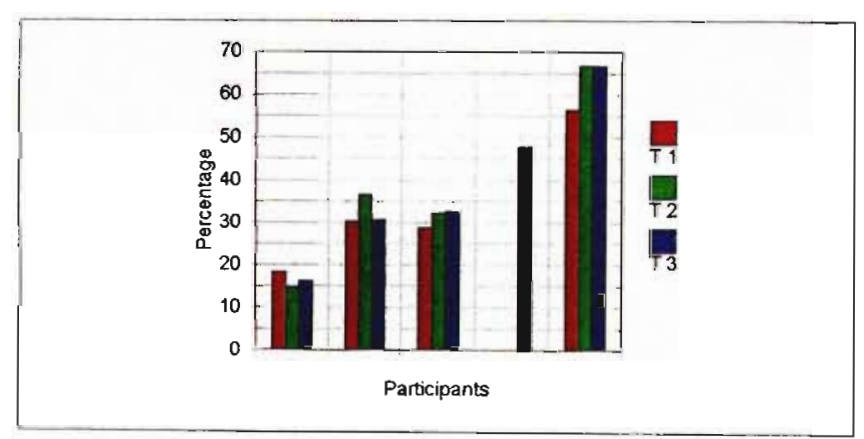

Figure 8e: CD $8 \%$ Across Time

$\begin{array}{rrrrrr} & 1 & 2 & 3 & 4 & 5 \\ \text { T 1 } & 354 & 684 & 1107 & & 654 \\ \text { T 2 } & 249 & 710 & 1510 & & 860 \\ \text { T 3 } & 305 & 644 & 1304 & 1117 & 872\end{array}$

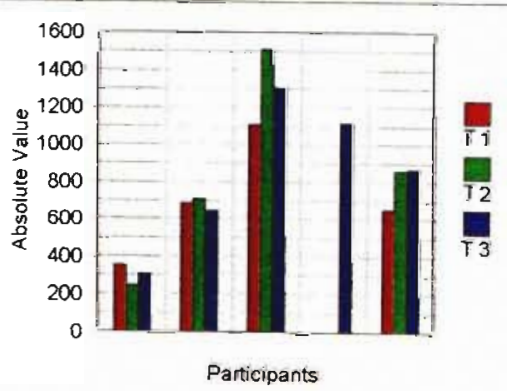

Figure 8f: CD 8 Absolute Across Time 


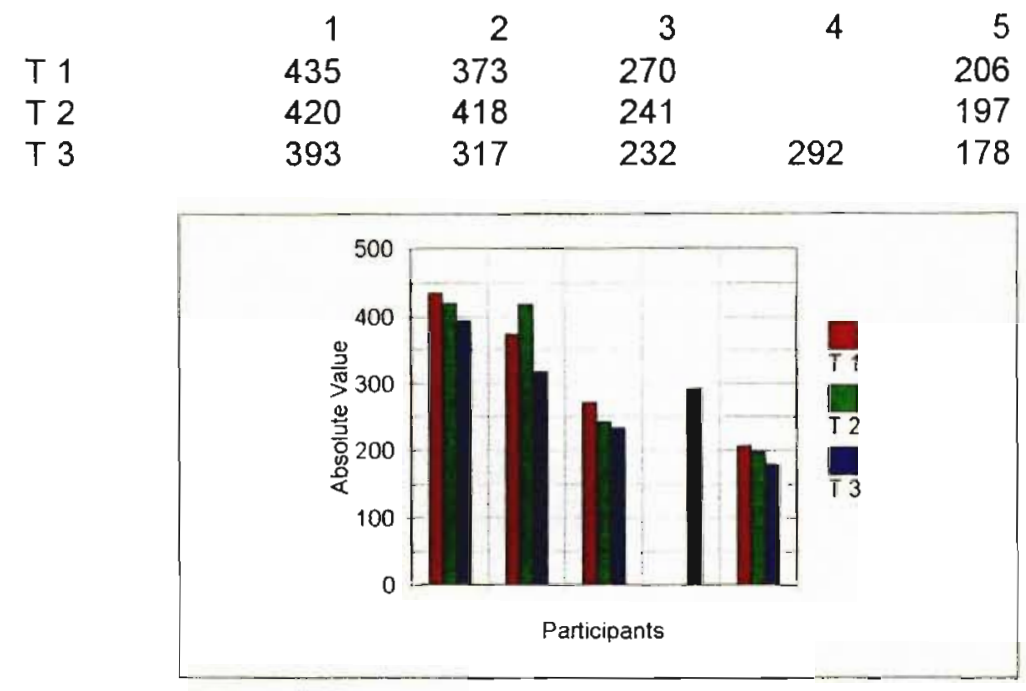

Figure 8g: Platelets Across Time

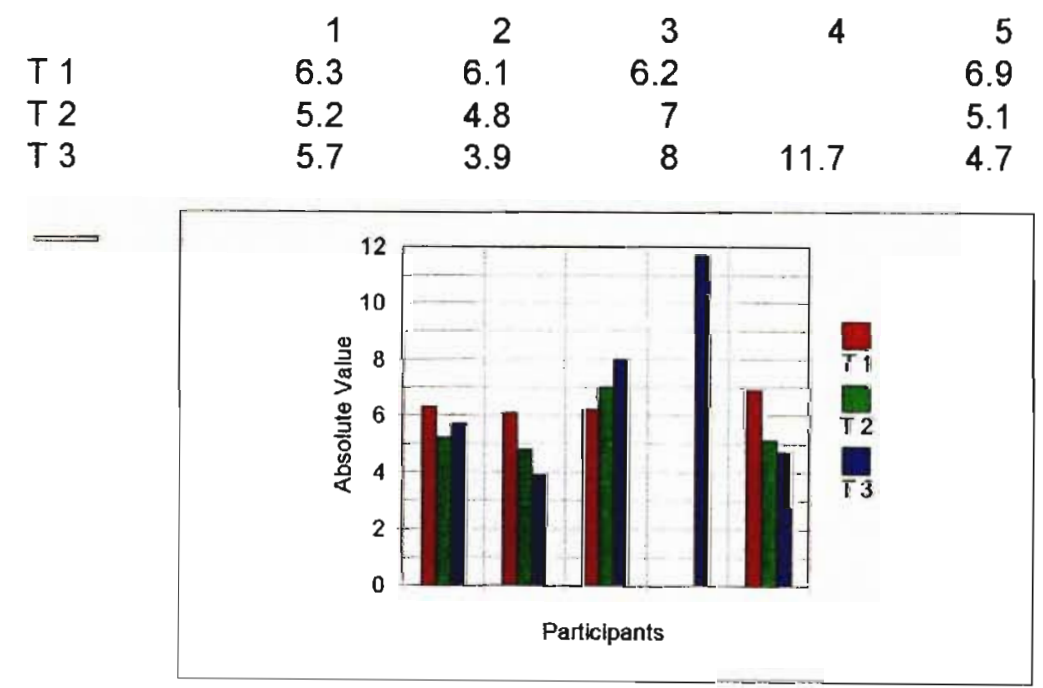

Figure 8h: White Cell Count Across Time

$\begin{array}{rrrrrr} & 1 & 2 & 3 & 4 & 5 \\ \text { T 1 } & 61.1 & 54 & 21 & & 73.3 \\ \text { T 2 } & 59.4 & 41.5 & 20 & & 55.6 \\ \text { T 3 } & 58.8 & 29 & 36 & 69.1 & 56.5\end{array}$

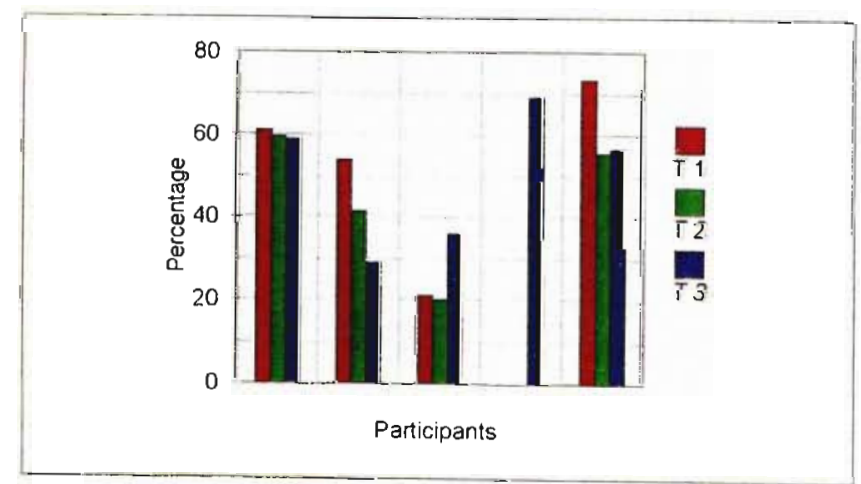

Figure 8i: Neutrophils \% Across Time 


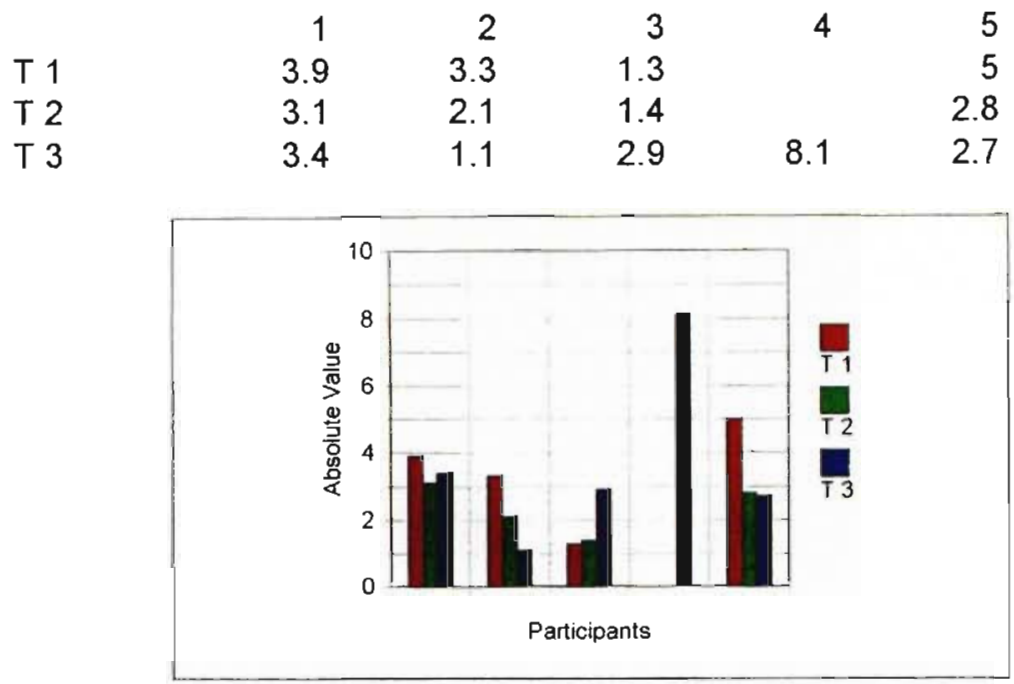

Figure 8j : Neutrophil Absolute Across Time

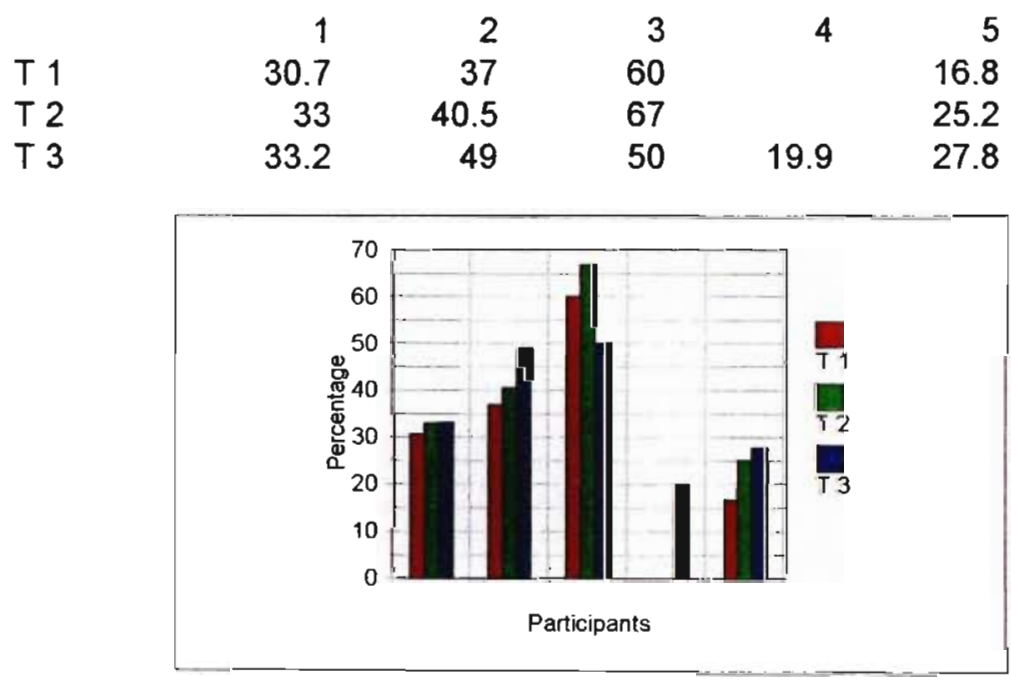

Figure 8k: Lymphocyte \% Across Time

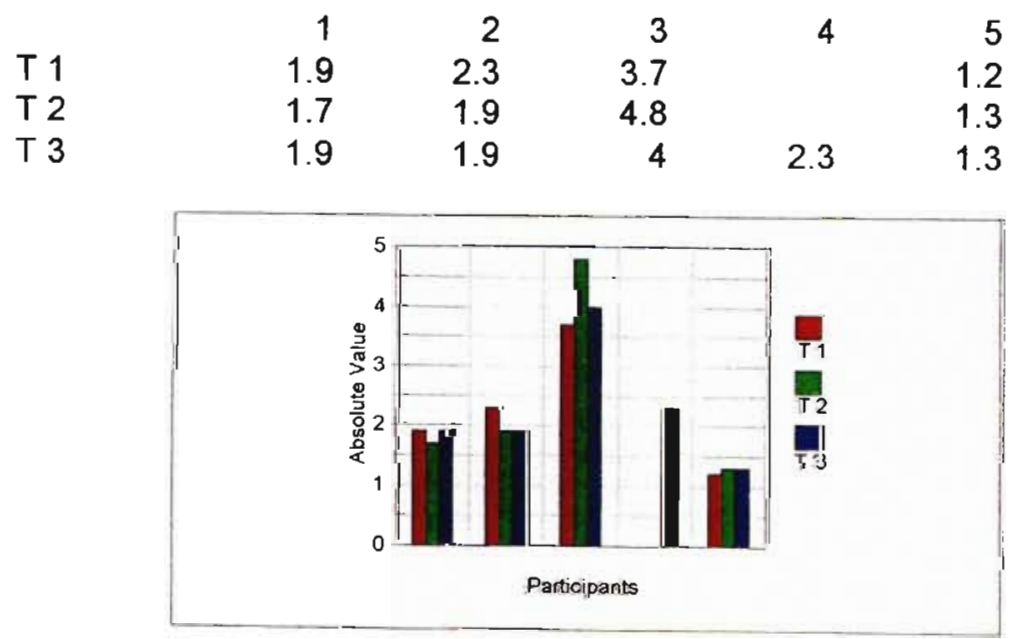

Figure 8I: Lymphocyte Absolute Across Time 


$\begin{array}{rrr}1 & 2 & 3 \\ 6.5 & 8 & 11\end{array}$

4 5

$\begin{array}{rrrr}6.5 & 8 & 11 & 8.7\end{array}$

$\begin{array}{llll}6.6 & 10.3 & 10 & 9.6\end{array}$

9

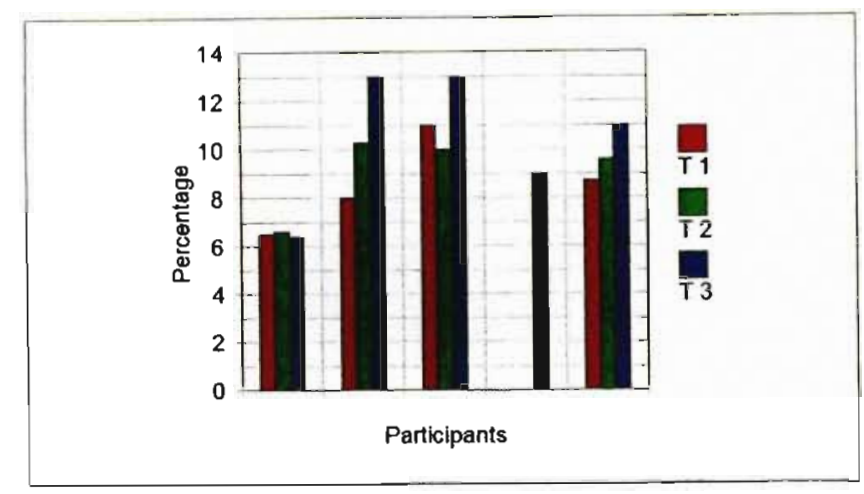

Figure 8m: Monocytes \% Across Time

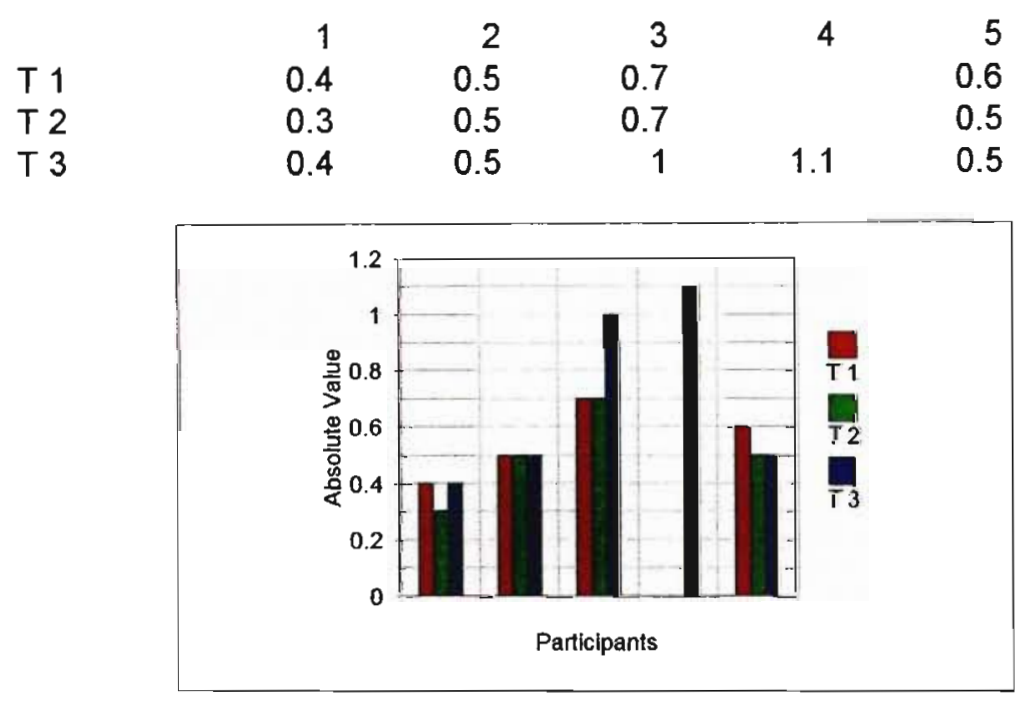

Figure 8n: Monocyte Absolute Across Time

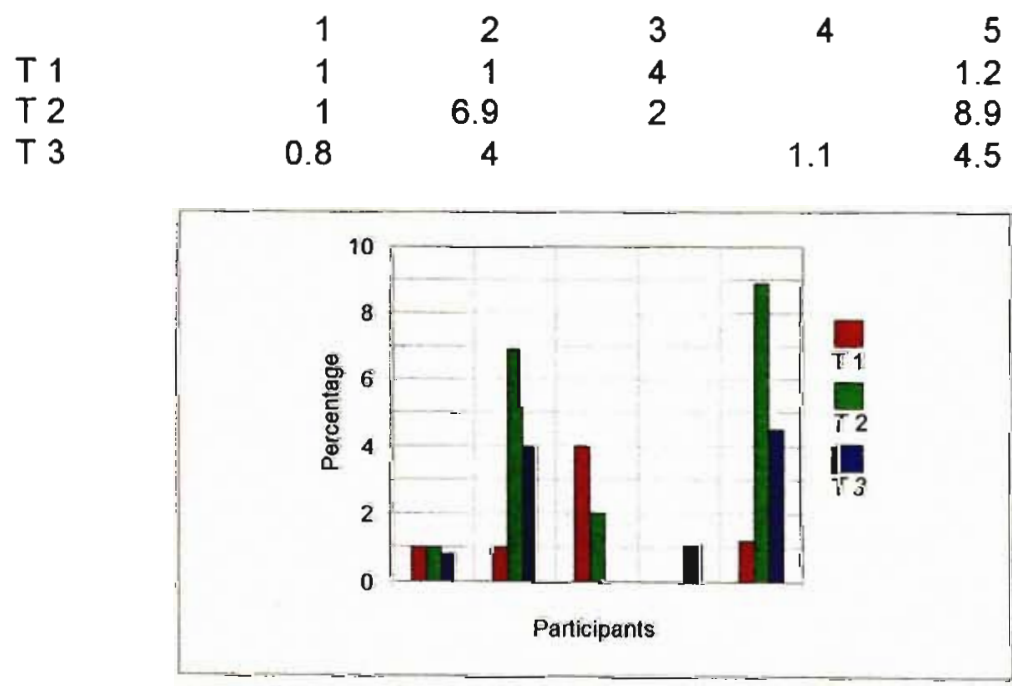

Figure 80: Eosinophil \% Across Time 


$\begin{array}{rrrrrr} & 1 & 2 & 3 & 4 & 5 \\ \text { T 1 } & 0.1 & 0.1 & 0.2 & & 0.1 \\ \text { T 2 } & 0.1 & 0.3 & 0.1 & & 0.5 \\ \text { T 3 } & 0 & 0.2 & & 0.1 & 0.2\end{array}$

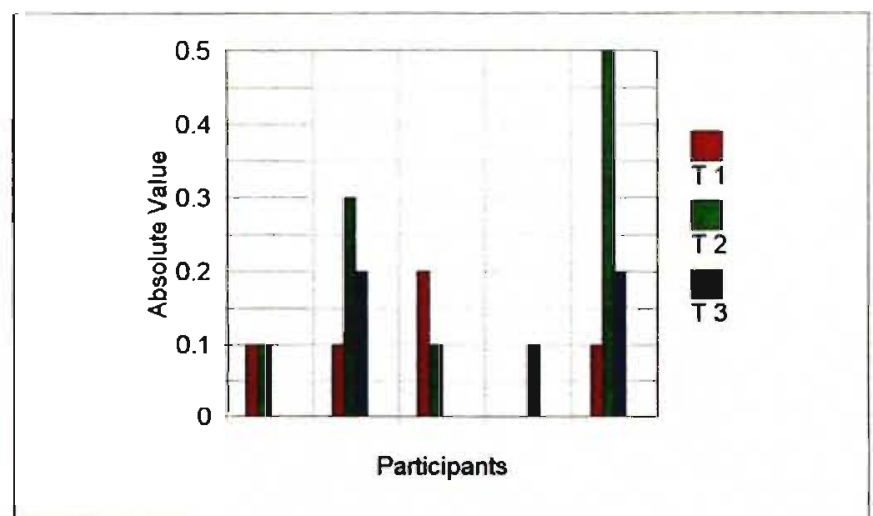

Figure 8p: Eosinophil Absolute Across Time 
Appendix 7 - Participants Psychological Measures Across Time 
Brief Symptom Inventory

Table 18a : Somatization Scale Across Time

\begin{tabular}{|c|c|c|c|c|c|}
\hline \multirow[t]{2}{*}{ Time } & \multicolumn{5}{|c|}{ Participants } \\
\hline & 1 & 2 & 3 & 4 & 5 \\
\hline 1 & 23 & 23 & 9 & 13 & 15 \\
\hline 2 & 24 & 21 & 24 & 7 & 21 \\
\hline 3 & 24 & 18 & 12 & 11 & 19 \\
\hline
\end{tabular}

Table 18b : Obsessive-Compulsive Scale Across Time

\begin{tabular}{|c|c|c|c|c|c|}
\hline \multirow[t]{2}{*}{ Time } & \multicolumn{5}{|c|}{ Participants } \\
\hline & 1 & 2 & 3 & 4 & 5 \\
\hline 1 & 24 & 24 & 13 & 21 & 11 \\
\hline 2 & 24 & 17 & 19 & 16 & 14 \\
\hline 3 & 24 & 18 & 14 & 15 & 16 \\
\hline
\end{tabular}

Table 18c :Interpersonal-Sensitivity Scale Across Time

\begin{tabular}{|l|l|l|l|l|l|}
\hline \multirow{2}{*}{ Time } & \multicolumn{6}{|c|}{ Participants } \\
\cline { 2 - 7 } & \multicolumn{1}{|c|}{1} & \multicolumn{1}{|c|}{2} & \multicolumn{1}{c|}{3} & \multicolumn{1}{c|}{4} & 5 \\
\hline 1 & 16 & 14 & 2 & 11 & 11 \\
\hline 2 & 16 & 12 & 16 & 13 & 14 \\
\hline 3 & 16 & 12 & 4 & 11 & 16 \\
\hline
\end{tabular}


Table 18d: Depression Scale Across Time

\begin{tabular}{|l|l|l|l|l|l|}
\hline \multirow{2}{*}{ Time } & \multicolumn{6}{|c|}{ Participants } \\
\cline { 2 - 7 } & \multicolumn{2}{|c|}{1} & \multicolumn{1}{|c|}{2} & \multicolumn{1}{c|}{3} & \multicolumn{1}{c|}{4} \\
\hline 1 & 24 & 24 & 0 & 24 & 17 \\
\hline 2 & 24 & 20 & 23 & 23 & 16 \\
\hline 3 & 24 & 17 & 7 & 18 & 17 \\
\hline
\end{tabular}

Table 18e : Anxiety Scale Across Time

\begin{tabular}{|l|l|l|l|l|l|}
\hline \multirow{2}{*}{ Time } & \multicolumn{6}{|c|}{ Participants } \\
\cline { 2 - 7 } & \multicolumn{2}{|c|}{1} & \multicolumn{1}{|c|}{2} & \multicolumn{1}{c|}{3} & \multicolumn{2}{c|}{4} & 5 \\
\hline 1 & 24 & 24 & 12 & 22 & 15 \\
\hline 2 & 24 & 23 & 20 & 22 & 20 \\
\hline 3 & 24 & 23 & 17 & 22 & 20 \\
\hline
\end{tabular}

Table 18f: Hostility Scale Across Time

\begin{tabular}{|c|c|c|c|c|c|}
\hline \multirow[t]{2}{*}{ Time } & \multicolumn{5}{|c|}{ Participants } \\
\hline & 1 & 2 & 3 & 4 & 5 \\
\hline 1 & 20 & 18 & 4 & 4 & 18 \\
\hline 2 & 24 & 10 & 14 & 11 & 16 \\
\hline 3 & 24 & 9 & 13 & 10 & 14 \\
\hline
\end{tabular}


Table 18g :Phobic-Anxiety Scale Across Time

\begin{tabular}{|l|l|l|l|l|l|}
\hline \multirow{2}{*}{ Time } & \multicolumn{5}{|c|}{ Participants } \\
\cline { 2 - 7 } & \multicolumn{2}{|c|}{1} & \multicolumn{1}{|c|}{2} & \multicolumn{1}{c|}{3} & \multicolumn{1}{c|}{4} \\
\hline 1 & 20 & 20 & 5 & 19 & 13 \\
\hline 2 & 20 & 20 & 20 & 19 & 16 \\
\hline 3 & 20 & 19 & 7 & 13 & 24 \\
\hline
\end{tabular}

Table 18h : Paranoid Ideation Scale Across Time

\begin{tabular}{|l|l|l|l|l|l|}
\hline \multirow{2}{*}{ Time } & \multicolumn{6}{|c|}{ Participants } \\
\cline { 2 - 7 } & \multicolumn{1}{|c|}{1} & \multicolumn{1}{|c|}{2} & \multicolumn{1}{c|}{3} & \multicolumn{1}{c|}{4} & \multicolumn{1}{c|}{5} \\
\hline 1 & 20 & 20 & 4 & 16 & 14 \\
\hline 2 & 20 & 18 & 20 & 9 & 20 \\
\hline 3 & 20 & 18 & 9 & 14 & 16 \\
\hline
\end{tabular}

Table 18i : Psychotism Scale Across Time

\begin{tabular}{|l|l|l|l|l|l|}
\hline \multirow{2}{*}{ Time } & \multicolumn{6}{|c|}{ Participants } \\
\cline { 2 - 7 } & \multicolumn{1}{|c|}{1} & \multicolumn{1}{|c|}{2} & \multicolumn{1}{c|}{3} & \multicolumn{1}{c|}{4} & \multicolumn{1}{c|}{5} \\
\hline 1 & 20 & 18 & 4 & 14 & 15 \\
\hline 2 & 20 & 16 & 20 & 13 & 20 \\
\hline 3 & 20 & 13 & 12 & 12 & 20 \\
\hline
\end{tabular}




\section{Revised Impact of Events Scale}

Table $18 \mathrm{j}$ : Intrusion Scale Across Time

\begin{tabular}{|l|l|l|l|l|l|}
\hline \multirow{2}{*}{ Time } & \multicolumn{5}{|c|}{ Participants } \\
\cline { 2 - 7 } & \multicolumn{1}{|c|}{1} & \multicolumn{2}{|c|}{2} & \multicolumn{1}{c|}{3} & \multicolumn{1}{c|}{4} \\
\hline 1 & 29 & 27 & 10 & 13 & 25 \\
\hline 2 & 16 & 31 & 30 & 29 & 35 \\
\hline 3 & 16 & 29 & 28 & 27 & 21 \\
\hline
\end{tabular}

Table 18k :Avoidance Scale Across Time

\begin{tabular}{|l|l|l|l|l|l|}
\hline \multirow{2}{*}{ Time } & \multicolumn{5}{|c|}{ Participants } \\
\cline { 2 - 7 } & \multicolumn{1}{|c|}{1} & \multicolumn{1}{|c|}{2} & \multicolumn{1}{c|}{3} & \multicolumn{1}{c|}{4} & \multicolumn{1}{c|}{5} \\
\hline 1 & 16 & 18 & 8 & 22 & 18 \\
\hline 2 & 20 & 19 & 38 & 26 & 33 \\
\hline 3 & 22 & 23 & 32 & 20 & 3 \\
\hline
\end{tabular}

\section{Ways of Coping}

Table 181 :Wishful thinking / Escape Scale Across Time

\begin{tabular}{|l|l|l|l|l|l|}
\hline \multirow{2}{*}{ Time } & \multicolumn{5}{|c|}{ Participants } \\
\cline { 2 - 7 } & \multicolumn{1}{|c|}{1.} & \multicolumn{1}{|c|}{2} & \multicolumn{1}{c|}{3} & 4 & 5 \\
\hline 1 & 31 & 29 & 39 & 32 & 60 \\
\hline 2 & 34 & 33 & 33 & 42 & 64 \\
\hline 3 & 33 & 32 & 33 & 31 & 61 \\
\hline
\end{tabular}


Table $18 \mathrm{~m}$ :Acceptance Scale Across Time

\begin{tabular}{|c|c|c|c|c|c|}
\hline \multirow[t]{2}{*}{ Time } & \multicolumn{5}{|c|}{ Participants } \\
\hline & 1 & 2 & 3 & 4 & 5 \\
\hline 1 & 26 & 37 & 31 & 31 & 34 \\
\hline 2 & 29 & 34 & 20 & 31 & 34 \\
\hline 3 & 29 & 31 & 29 & 27 & 35 \\
\hline
\end{tabular}

Table 18n : Emotional-withholding Scale Across Time

\begin{tabular}{|l|l|l|l|l|l|}
\hline \multirow{2}{*}{ Time } & \multicolumn{5}{|c|}{ Participants } \\
\cline { 2 - 7 } & \multicolumn{1}{|c|}{1} & \multicolumn{1}{|c|}{2} & \multicolumn{1}{c|}{3} & \multicolumn{1}{c|}{4} & 5 \\
\hline 1 & 21 & 21 & 25 & 20 & 20 \\
\hline 2 & 22 & 20 & 28 & 18 & 20 \\
\hline 3 & 22 & 29 & 17 & 23 & 20 \\
\hline
\end{tabular}

Table 180: Problem-focused / Help-seeking Scale Across Time

\begin{tabular}{|l|l|l|l|l|l|}
\hline \multirow{2}{*}{ Time } & \multicolumn{6}{|c|}{ Participants-167- } \\
\cline { 2 - 7 } & \multicolumn{1}{|c|}{1} & \multicolumn{2}{|c|}{2} & \multicolumn{1}{c|}{3} & \multicolumn{1}{c|}{4} \\
\hline 1 & 30 & 32 & 29 & 31 & 52 \\
\hline 2 & 26 & 38 & 11 & 34 & 52 \\
\hline 3 & 25 & 39 & 28 & 24 & 52 \\
\hline
\end{tabular}


Table 18p : Self Blame Scale Across Time

\begin{tabular}{|l|l|l|l|l|l|}
\hline \multirow{2}{*}{ Time } & \multicolumn{5}{|c|}{ Participants } \\
\cline { 2 - 7 } & \multicolumn{2}{|c|}{1} & \multicolumn{1}{|c|}{2} & \multicolumn{1}{c|}{3} & \multicolumn{1}{c|}{4} \\
\hline 1 & 20 & 20 & 24 & 18 & 72 \\
\hline 2 & 20 & 20 & 17 & 27 & 72 \\
\hline 3 & 20 & 23 & 26 & 23 & 72 \\
\hline
\end{tabular}

Table 18 q: Growth Scale Across Time

\begin{tabular}{|c|c|c|c|c|c|}
\hline \multirow[t]{2}{*}{ Time } & \multicolumn{5}{|c|}{ Participants } \\
\hline & 1 & 2 & 3 & 4 & 5 \\
\hline 1 & 15 & 13 & 18 & 13 & 28 \\
\hline 2 & 12 & 15 & 14 & 22 & 28 \\
\hline 3 & 12 & 10 & 18 & 17 & 27 \\
\hline
\end{tabular}

Table 18r : Help-seeking Scale Across Time

\begin{tabular}{|l|l|l|l|l|l|}
\hline \multirow{2}{*}{ Time } & \multicolumn{6}{|c|}{ Participants } \\
\cline { 2 - 7 } & \multicolumn{1}{|c|}{1} & \multicolumn{1}{|c|}{2} & \multicolumn{1}{c|}{3} & \multicolumn{1}{c|}{4} & 5 \\
\hline 1 & 9 & 8 & 8 & 13 & 16 \\
\hline 2 & 7 & 11 & 2 & 12 & 16 \\
\hline 3 & 7 & 11 & 9 & 11 & 16 \\
\hline
\end{tabular}


Duke-UNC Health Profile

Table 18s : Symptom Status Scale Across Time

\begin{tabular}{|c|c|c|c|c|c|}
\hline \multirow[t]{2}{*}{ Time } & \multicolumn{5}{|c|}{ Participants } \\
\hline & 1 & 2 & 3 & 4 & 5 \\
\hline 1 & 30 & 10 & 28 & 14 & 15 \\
\hline 2 & 27 & 28 & 29 & 30 & 7 \\
\hline 3 & 41 & 23 & 29 & 40 & 10 \\
\hline
\end{tabular}

Table $18 \mathrm{t}$ : Physical Function Scale Across Time

\begin{tabular}{|c|c|c|c|c|c|}
\hline \multirow[t]{2}{*}{ Time } & \multicolumn{5}{|c|}{ Participants } \\
\hline & 1 & 2 & 3 & 4 & 5 \\
\hline 1 & 15 & 21 & 22 & 15 & 23 \\
\hline 2 & 31 & 16 & 23 & 11 & 24 \\
\hline 3 & 31 & 18 & 23 & 6 & 24 \\
\hline
\end{tabular}

Table 18 u: Emotional Function Scale Across Time

\begin{tabular}{|c|c|c|c|c|c|}
\hline \multirow[t]{2}{*}{ Time } & \multicolumn{5}{|c|}{ Participants } \\
\hline & 1 & 2 & 3 & 4 & 5 \\
\hline 1 & 64 & 70 & 69 & 70 & 26 \\
\hline 2 & 66 & 60 & 74 & 74 & 0 \\
\hline 3 & 57 & 57 & 75 & 72 & 20 \\
\hline
\end{tabular}


Table 18v : Social Function Scale Across Time

\begin{tabular}{|c|c|c|c|c|c|}
\hline \multirow[t]{2}{*}{ Time } & \multicolumn{5}{|c|}{ Participants } \\
\hline & 1 & 2 & 3 & 4 & 5 \\
\hline 1 & 6 & 10 & 7 & 4 & 2 \\
\hline 2 & 4 & 9 & 8 & 5 & 2 \\
\hline 3 & 4 & 8 & 8 & 7 & 2 \\
\hline
\end{tabular}


Appendix 8 - Table 19: Mean Scores and Standard Deviations of

Psychological Measures Across Time 1,2 and 3 
Table 19 : Mean Scores and Standard Deviations of Psychological measures Across Time

\begin{tabular}{|c|c|c|c|c|c|c|}
\hline \multirow[t]{2}{*}{ Scale } & \multicolumn{2}{|c|}{ Time 1} & \multicolumn{2}{|c|}{ Time 2} & \multicolumn{2}{|c|}{ Time 3} \\
\hline & mean score & $\mathrm{SD}$ & mean score & $\mathrm{SD}$ & mean score & $\mathrm{SD}$ \\
\hline \multicolumn{7}{|c|}{ Brief Symptom Inventory } \\
\hline Somatization & 16.35 & 6.079 & 19.4 & 5.5408 & 16.25 & 5.7155 \\
\hline Obsess.-compul & 20.0 & 10.69 & 20.0 & 3.8079 & 18.6 & 4.9800 \\
\hline Interpers.-Sens. & 10.7 & 4.966 & 14.2 & 1.6330 & 9.6 & 5.0299 \\
\hline Depression & 17.7 & 10.402 & 21.5 & 1.6733 & 16.5 & 6.1074 \\
\hline Anxiety & 19.4 & 5.5509 & 21.7 & 2.0656 & 20.4 & 2.3022 \\
\hline Hostility & 12.8 & 9.6540 & 14.2 & 3.4303 & 13.2 & 4.3243 \\
\hline Phobic-Anxiety & 15.4 & 6.5038 & 19.0 & 1.7321 & 17 & 5.3541 \\
\hline Psychotism & 14.2 & 6.6068 & 17.7 & 2.9944 & 15.6 & 4.0373 \\
\hline Paranoid Idea. & 15.7 & 6.4041 & 17.7 & 1.0954 & 15.3 & 4.2190 \\
\hline \multicolumn{7}{|l|}{ Impact of Events } \\
\hline Avoidance & 17 & 5.220 & 27 & 7.5565 & 20.0 & 10.559 \\
\hline Intrusion & 21 & 8.6747 & 28 & 7.0711 & 24.2 & 5.5408 \\
\hline \multicolumn{7}{|l|}{ Ways of Coping } \\
\hline Wish /Escape & 36.6 & 12.880 & 37.8 & 6.5115 & 37 & 7.9057 \\
\hline Prob.Foc/Help & 34.7 & 9.6805 & 33.6 & 7.0427 & 31.2 & 7.4297 \\
\hline Emotional-with. & 24.4 & 3.9433 & 23.8 & 3.6742 & 24.8 & 4.8166 \\
\hline Self-Blame & 20.5 & 25.793 & 23.3 & 3.4448 & 23.55 & 2.8810 \\
\hline Acceptance & 30.4 & 4.4385 & 28.6 & 3.6697 & 28.4 & 5.0794 \\
\hline Help-Seeking & 9.6 & 3.8080 & 9.4 & 2.0976 & 9.0 & 1.8708 \\
\hline \multicolumn{7}{|c|}{ Duke-UNC Health Profile } \\
\hline Symptom & 20.0 & 9.0139 & 28.9 & 8.8713 & 30.0 & 11.358 \\
\hline Emotional & 60.2 & 19.063 & 62.4 & 17.603 & 58.2 & 20.241 \\
\hline Physical & 19.1 & 3.9386 & 21.2 & 8.8318 & 20.4 & 9.2898 \\
\hline Social & 5.8 & 3.0332 & 6.2 & 2.6394 & 6.4 & 2.5100 \\
\hline
\end{tabular}

